\title{
Initial Evaluation of SAR Capabilities in UAV Multicopter Platforms
}

\author{
Marc Lort, Albert Aguasca, Member, IEEE, Carlos López-Martínez, Senior Member, IEEE, \\ and Tomás Martínez Marín
}

\begin{abstract}
Airborne synthetic aperture radar (SAR) sensors have been commonly used during the last decades to monitor different phenomena in medium-scale areas of observation, such as object detection and characterization or topographic mapping. The use of unmanned aerial vehicles (UAVs) is a cost-effective solution that offers higher operational flexibility than airborne systems to monitor these types of scenarios. The Universitat Politècnica de Catalunya has developed the first fully polarimetric SAR system at X-band integrated into a small UAV multicopter platform (UAV MP). The sensor, called AiR-based remote sensing, has been integrated into the platform overcoming restrictions of weight, space, robustness, and power consumption. The present paper introduces the SAR capabilities of UAV MPs, which open new unexploited potentials to airborne SAR. To demonstrate the validity of the developed system, some measurement campaigns have been conducted in the outskirts of Barcelona, Spain.
\end{abstract}

Index Terms-Airborne synthetic aperture radar (SAR), unmanned aerial vehicle (UAV) multicopter, UAV SAR.

\section{INTRODUCTION}

$\mathbf{S}$ YNTHETIC aperture radar (SAR) sensors allow a flexible surface observation in innumerous applications areas such as agriculture, forestry, topography, earth-resource mapping, or environmental monitoring [1]-[3]. Unmanned aerial vehicles (UAVs) represent an alternative to be considered in airborne remote sensing in terms of cost effectiveness and operational flexibility. Nevertheless, these platforms have some limitations when used in SAR applications. One of the most important is the undesired deviations from the nominal flight trajectory that may cause defocusing, geometric distortions, or phase errors in the SAR images.

The purpose of this work is to validate the system performance and to evaluate the SAR capabilities in UAV multicopter

Manuscript received February 2, 2017; revised May 22, 2017 and July 17, 2017; accepted September 5, 2017. This work was supported by the Spanish Ministry of Science and Innovation (MICINN) under Project TIN201455413C21P and Project TIN201455413C22P, the Research Grant BES-2012-060089, and the Unidad de Excelencia Maria de Maeztu MDM2016-0600, which is financed by the Agencia Estatal de Investigación, Spain. (Corresponding author: Marc Lort.)

M. Lort, A. Aguasca, and C. López-Martínez are with the CommSensLab, Universitat Politècnica de Catalunya, 08034 Barcelona, Spain (e-mail: marc.lort@tsc.upc.edu; aguasca@tsc.upc.edu; carlos.lopez@tsc.upc.edu).

T. M. Marín is with the Department of Physics, System Engineering and Signal Theory, University of Alicante, E-03690 Alicante, Spain (e-mail: tomas.martinez@ua.es).

Color versions of one or more of the figures in this paper are available online at http://ieeexplore.ieee.org.

Digital Object Identifier 10.1109/JSTARS.2017.2752418

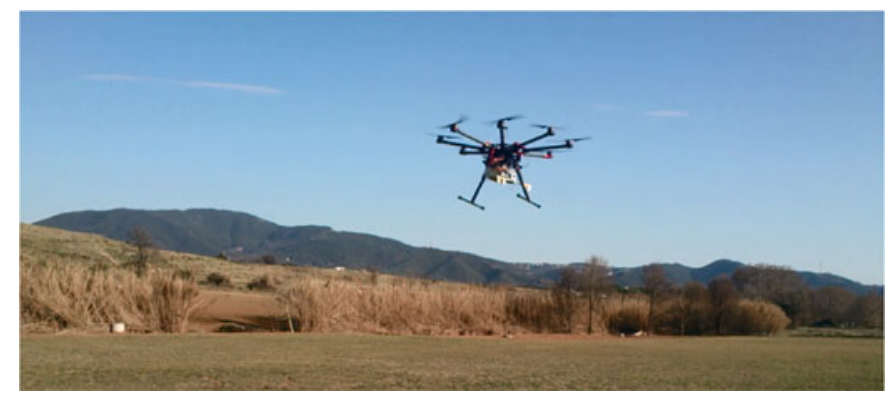

Fig. 1. Fully polarimetric ARBRES-X SAR integrated in the UAV MP.

platforms (UAV MP), such as repeat-pass interferometry, polarimetric measurements, and vertical or circular trajectories that can be used, for instance, for tomographic purposes. The use of this platform introduces new possibilities in airborne SAR observation due to its inherent flight capabilities and characteristics.

The present paper is organized as follows. Section II presents the system description, the architecture, and the setting parameters of the ARBRES-X SAR sensor. Furthermore, the UAV MP used to integrate the ARBRES-X SAR sensor is introduced, and its main advantages and disadvantages are presented. In Section III, the experimental results are assessed. The stability of the platform is evaluated as a function of the aperture length, and an autofocus algorithm is applied to improve the quality of the retrieved images. Moreover, different innovative flight strategies for airborne SAR observation are introduced, such as vertical flights, circular flights, or repeat-pass flights for interferometry. Finally, the main conclusions and major remarks are given in Section IV.

\section{UAV MP AND SAR SYSTEM DESCRIPTION}

In this section, the main components and characteristics of the integrated system are presented. The different elements are the ARBRES-X SAR sensor, the UAV MP, and the inertial measurement unit (IMU). Fig. 1 shows a picture of the complete system flying during a measurement campaign.

\section{A. UAV Multicopter Platform}

The Spreading Wings S1000 from DJI, designed for professional aerial photography and cinematography, has been chosen to integrate the ARBRES-X SAR sensor. UAV MPs have several advantages compared with a fixed-wing airplane UAV that 
makes them suitable to integrate a sensor to perform SAR measurements. The main advantage of the UAV MP is that it does not need a long landing strip. Due to its vertical lift capability, it only needs a small area, such as a forest clearing, to take off and to land, making it operational in almost any location. Besides, its capacity to hover and perform agile maneuvering makes this type of platforms well suited for many applications in airborne SAR observation. Some examples could be object detection or urban structure characterization, where precision flights and the ability to maintain the observation on a single target for extended periods of time may be required.

Another important advantage is that the UAV MP can perform 3-D flights due to its ability to move in almost any direction, opening a wide range of new possibilities in airborne SAR measurements. Vertical and circular flights can be combined, for example, to define new strategies to obtain 3-D measurements of structures. Vertical flights can be used to perform vertical apertures and also to think about high-resolution tomography. On the other hand, circular flights can be used, for instance, to obtain holographic/tomographic SAR images. Finally, some preprogrammed flights with autotrigger can be performed with the autopilot function of the UAV MP. It is important because it opens the possibility to predefine different strategies of flight, such as repeat-pass interferometry in airborne SAR, with a good control of the route and the measurement time. All these advantages make the UAV MP a versatile platform that gives a greater degree of operational flexibility with respect to the conventional UAVs.

Despite the important advantages of the UAV MP, some drawbacks have also to be pointed out. It is inherently unstable if compared with the fixed-wing UAVs, so its capability to perform constant-velocity straight linear trajectories is reduced. This is extremely important because errors in positioning and velocity of the platform during the acquisition time affect drastically to the measurements performed with the ARBRES-X SAR sensor. In this context, Fig. 2 is intended to provide a graphical description of the effects of having nonuniform trajectories during the realization of the measurements.

Fig. 2(a) shows the example of a geometry considering an ideal flight path and a flight with deviations in height from the nominal track. In Fig. 2(b) and (c), the focusing of a permanent scatterer (PS) has been simulated considering an ideal flight path and a flight path with deviations in height. The focused singlelook complex (SLC) image of the PS obtained with the nonideal flight presents an important defocusing in the azimuth direction due to the deviations of the platform from the nominal track. For this reason, these effects must be compensated for when focusing the images, taking into account that the deviations from the ideal track can be present in the three spatial coordinates $x$, $y$, and $z$.

Another disadvantage of the UAV MP is the short flight autonomy, with respect to the fixed-wing UAVs, which reduces the total area that can be covered. The flight time, which depends on the power consumption of the system, is related to the total weight of the platform. This imposes several restrictions in weight for the integration of the ARBRES-X SAR sensor and

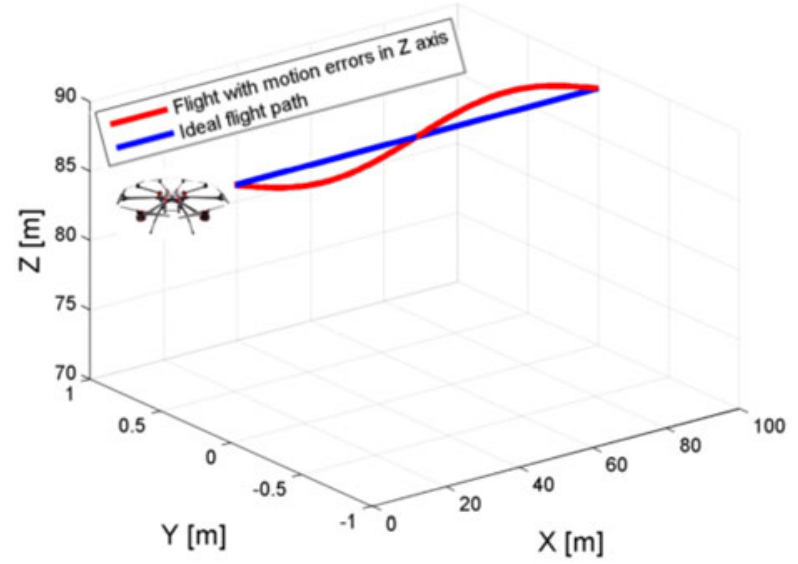

(a)

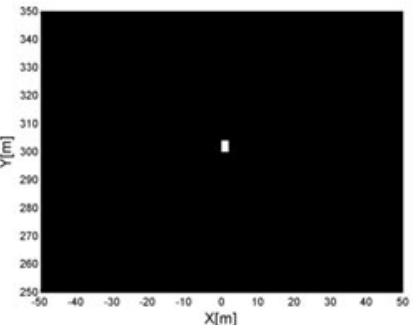

(b)

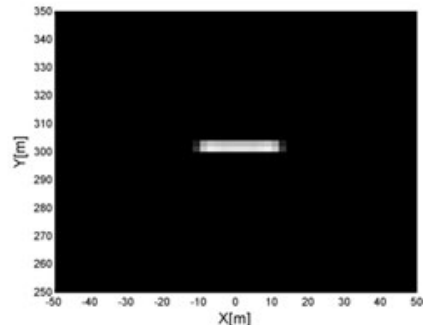

(c)
Fig. 2. (a) Representation of the geometry of a nonideal flight path with deviations in the $z$-axis. (b) SLC of a simulated target of an ideal flight path (c) SLC of a simulated target of a nonideal flight path with deviations in the $z$-axis.

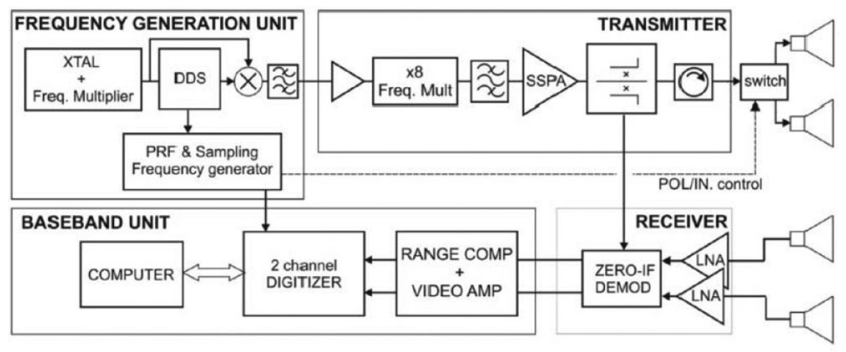

Fig. 3. ARBRES-X system architecture.

all the additional electronics, such as the trigger control or the IMU.

The whole system requires the integration of the ARBRES-X SAR sensor, the RF front-end bar, the IMU, the electronics of control, and the batteries into the UAV MP platform without exceeding the maximum takeoff weight. The SAR sensor, with a weight of approximately $2 \mathrm{~kg}$, has been placed in the lower part of the UAV MP together with the batteries of the platform. The total payload is around $6.5 \mathrm{~kg}$, and the whole integrated system weights around $10.5 \mathrm{~kg}$, slightly below the maximum takeoff weight of $11 \mathrm{~kg}$.

\section{B. ARBRES-X SAR Sensor}

The ARBRES-X SAR system represents an evolution of a 135 previous version developed by the Remote Sensing Laboratory 


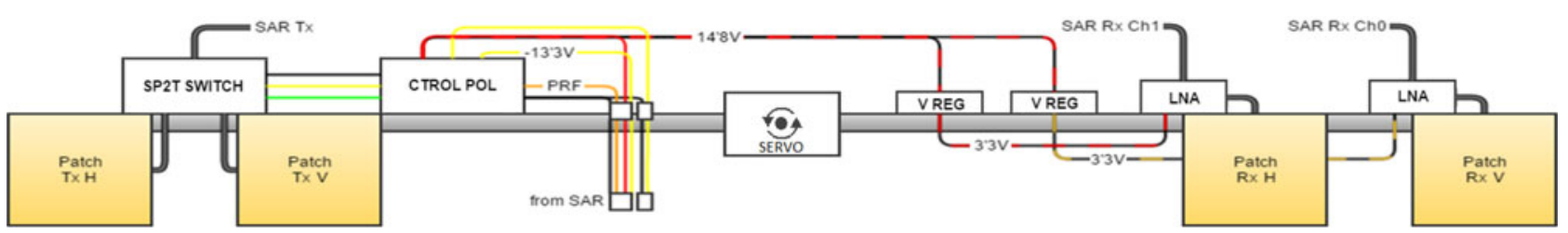

Fig. 4. Schematic of the transmitter/receiver RF front end.

TABLE I

ARBRES-X SAR SENSOR PARAMETERS

\begin{tabular}{lc}
\hline \hline System Parameters & X-Band \\
\hline Carrier Frequency $\left(f_{0}\right)$ & $9.65 \mathrm{GHz}$ \\
Chirp Sampling Frequency $\left(f_{s}\right)$ & $44.62 \mathrm{MHz}$ \\
Chirp P RF & $5.44 \mathrm{KHz}$ \\
Chirp Bandwidth & $100 \mathrm{MHz}$ \\
Transmitted Power & $30 \mathrm{dBm}$ \\
Range Resolution (nominal) & $1.5 \mathrm{~m}$ \\
Weight & $\approx 2 \mathrm{~kg}$ \\
Power Consumption & $29.3 \mathrm{~W}$ \\
\hline
\end{tabular}

of the UPC [4], [5]. It is a SAR sensor operating at $9.65 \mathrm{GHz}$ that works with a stepped-linear-frequency-modulated continuouswave signal. The complete system has been designed to be fitted in small UAVs, as is the case of the UAV MP. This has imposed strong constraints in its design in terms of weight, power consumption, compactness, and robustness. The main characteristics of the ARBRES-X SAR sensor are summarized in Table I, and the block diagram of the system is shown in Fig. 3.

The sensor can be divided into four units: the frequency generation unit (FGU), the transmitter, the receiver, and the baseband unit. The fully polarimetric capability is achieved with an array of $2 \times 2$ patch antennas transmitting and receiving in both vertical and horizontal channels. The microstrip antennas present a beamwidth of approximately $36^{\circ}$ in elevation and $42^{\circ}$ in azimuth. A solid-state switch is used to switch between the two transmitting antennas, changing the polarization for every transmitted chirp signal. Fig. 4 shows the RF front end, consisting of a lightweight aluminum bar together with the patch antennas, which has been specifically designed to be integrated into the UAV MP. In the image, the abbreviations SP2T and VREG correspond to single-pole double-throw and voltage regulator, respectively.

The receiver unit takes advantage of the use of the dechirpon-receive technique to reduce its complexity [6]. It consists of two parallel low-noise chains with a direct zero-IF demodulator, where a sample of the transmitted signal is used as a local oscillator. To reduce the noise and to amplify the signal in the receiver, a low-noise amplifier is directly connected to the antenna. After the baseband conversion, the signal acquisition in the baseband unit is performed by a commercial high-speed digitizer controlled by a single-board computer with a solid-state hard disk drive. The two baseband signals are synchronously digitized using trigger and clock references that are coherently generated in the FGU.

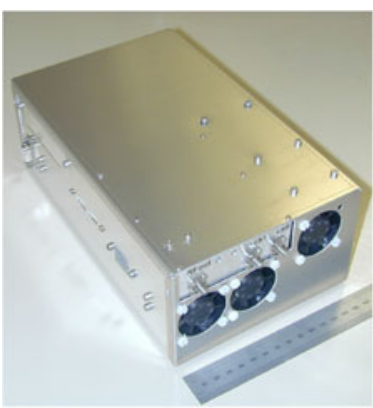

(a)

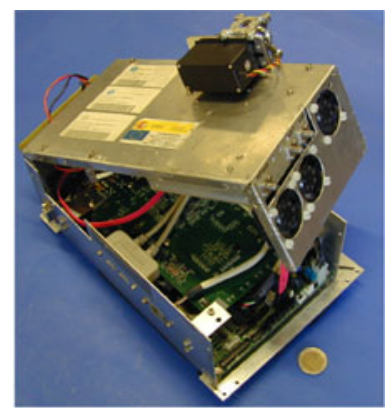

(b)

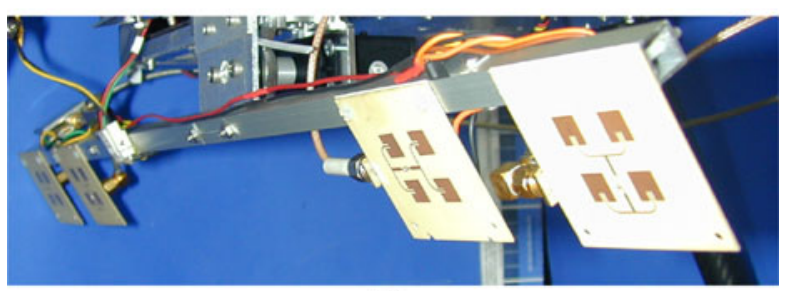

(c)

Fig. 5. (a) and (b) ARBRES-X SAR sensor. (c) Lightweight aluminum bar together with the patch antennas.

Fig. 5 shows the compacted lightweight ARBRES-X SAR and the transmitter and receiver antennas integrated in the system. A servo motor, together with a simple IMU, holds the lightweight aluminum bar. Its purpose is to maintain the antennas in horizontal position with respect to the ground, compensating the tilt angle of the platform while flying.

The area illuminated by the ARBRES-X SAR sensor is extended to approximately $1500 \mathrm{~m}$ in range and $900 \mathrm{~m}$ in azimuth The SLC images obtained from the retrieved data are processed offline with the backprojection algorithm (BPA) [7]. In this context, the received baseband signal is subsequently range compressed applying the fast Fourier transform, and then, the BPA algorithm is applied in order to perform the azimuth focusing. In spite of its high computational cost, the BPA offers a high degree of flexibility in focusing extended images from arbitrary synthetic apertures, limited either by antenna beam, the linear track, or by the data acquisition capacity [4]. Finally, the range resolution obtained with the parameters used by the ARBRES-X SAR sensor is approximately $1.5 \mathrm{~m}$, and the cross-range resolution depends on the type of measurement.

\section{UAV MP SAR EXPERIMENTAL RESULTS}

In this section, preliminary results obtained with the 193 ARBRES-X SAR sensor integrated in the UAV MP are 194 


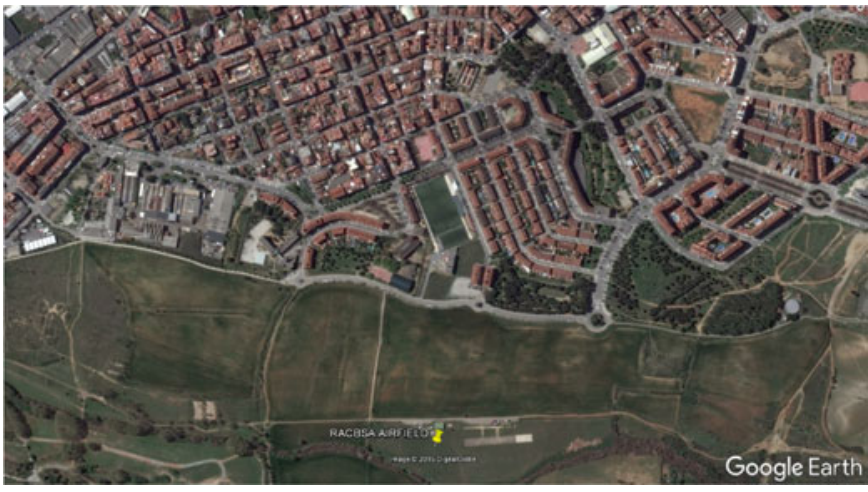

Fig. 6. Aerial photograph of the scenario from Google Earth.

presented. The aim is to explore the SAR capabilities of this type of platforms.

\section{A. Test Site Description}

The scenario selected to test the system and to perform several measurements is the Real Aero Club BarcelonaSabadell (RACBSA) Radio Control Airfield (Montcada i Reixac, Barcelona, Spain). The scenario contains agricultural fields surrounded by an urban area with smooth topography, as shown in Fig. 6. It is mainly composed of elements with high reflectivity, such as buildings, roads, pylons, or parked cars, which make it suitable to validate the system performances.

\section{B. Stability of the UAV MP SAR Platform and Application of the Autofocus Algorithm}

In Section II, the inherent instability of the platform has been mentioned, which can degrade the quality of the retrieved data from the ARBRES-X SAR sensor. The deviations respect to the nominal flight track impose severe restrictions on the maximum processable aperture length. The larger the aperture, the greater the cumulative positioning error of the platform with respect to the ideal flight path, and the greater the degree of defocusing in the retrieved images. Fig. 7 shows a comparison of three SLC images of the scenario obtained with different aperture lengths. Cartesian coordinates are used because it is visually easier to compare the SLC images with the real scenario. As it is evidenced, the defocusing increases for larger apertures, where the flight path is more susceptible to have deviations from the nominal trajectory. Considering the coordinate system presented in Fig. 2(a), an error in the positioning in the $x$-axis is due to errors in the velocity of the platform, which is equivalent to a nonuniform azimuth sampling of the signal. Errors in the $z$-axis are related to the height of the platform and in the $y$-axis with the lateral deviation of the platform with respect to the scenario. The defocusing of the image in Fig. 7(c) can be appreciated in the loss of resolution of the three PSs corresponding to polarimetric active radar calibrators (PARCs) located in the near range center of the image, together with the blurring effect in far range.

The aperture length is related to the azimuth resolution of the SAR system and to the signal-to-clutter ratio (SCR) of the focused images, since the energy emitted is greater for larger 233 apertures. Thus, in order to process larger apertures, it is necessary to compensate for the undesired effects of the nonidealities in the platform flight path. In this context, the first attempt was to incorporate an IMU integrated with a global positioning satellite (GPS) receiver that has a position accuracy of $2.5 \mathrm{~m}$ and an angular dynamic accuracy of $1^{\circ}$ RMS. The position and attitude information of the platform were logged simultaneously with the radar raw data for a proper image geocoding. The information provided by the IMU was intended to be used to apply the motion compensation (MoCo) technique in the focusing process. Thus, the possible motion errors could be compensated, and the defocusing, the geometric distortions, and the phase errors of the retrieved images could be minimized. Nevertheless, this option was discarded because the accuracy of cost-effective devices that are suitable to be integrated in the UAV MP, overcoming restrictions of size and weight, is not enough to fulfill the requirements needed. In this sense, the accuracy of these systems is too low in terms of the wavelength. Besides, the vibrations of the platform are another important drawback that degrades the quality of the measurements performed by the IMU.

To overcome the defocusing problem due to the instabilities of the UAV MP when processing long apertures, an autofocus algorithm has been applied. Different techniques have been proposed in the literature that are used to compensate for motion errors in SAR imagery [8], [9]. In our case, an algorithm based on the retrieved phase history of three PSs is applied with the objective to derive the deviations of the platform in the three axes [10]. Since it is difficult to find three natural PSs in the scenario with good reflectivity response, three different PARCs have been placed in visible and accessible locations of the scenario in order to validate the autofocus algorithm. Fig. 8 shows one of the PARCs placed in the agricultural field of the scenario, surrounded of growing vegetation. Their pointing angles (azimuth and elevation) are adjusted to maximize their visibility with the sensor. They can be recognized in Fig. 7 as the three PSs forming a triangle between 150 and $220 \mathrm{~m}$ in the range axis. The phase evolution of one PARC along the aperture can be expressed as

$$
\varphi_{\mathrm{PARC}}(t)=\frac{4 \pi R_{\mathrm{PARC}}(t)}{\lambda}
$$

where $\lambda$ is the wavelength and $R_{\text {PARC }}(t)$ is the slant range dis- 272 tance from the sensor to the PARC during the slow time $t$, i.e., the 273 distance from the sensor to the PARC for every antenna position 274 during the acquisition time. Analyzing the evolution of the phase 275 of the PARC $\varphi_{\text {PARC }}(t)$ during the acquisition time, the relative 276 distance variation from the sensor to the PARC $R_{\mathrm{PARC}}(t)$ can 277 be obtained from (1), but not the nominal value of the distance. 278 In practice, it can be extrapolated for the entire aperture know- 279 ing the slant range distance at the first instant of measurement 280 $R_{\text {PARC }}(t=0)$. Despite not having the real value $R_{\text {PARC }}(t=0), \quad 281$ it can be approximated by its ideal value taking as reference the 282 position of the platform given by the GPS. This approximation 283 is valid because the positioning errors of the platform are small 284 in comparison with the distance from the sensor to the scatterer. 285 


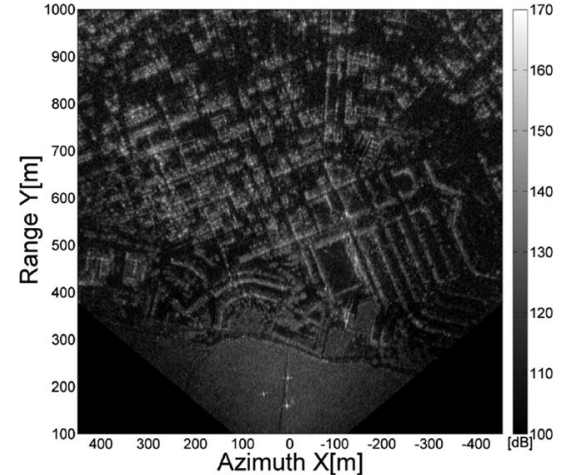

(a)

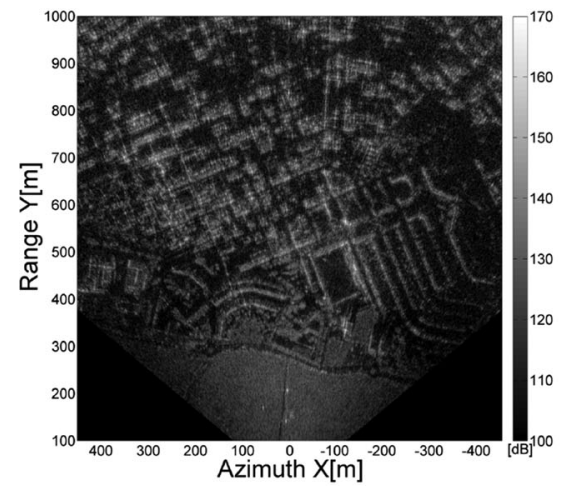

(d)

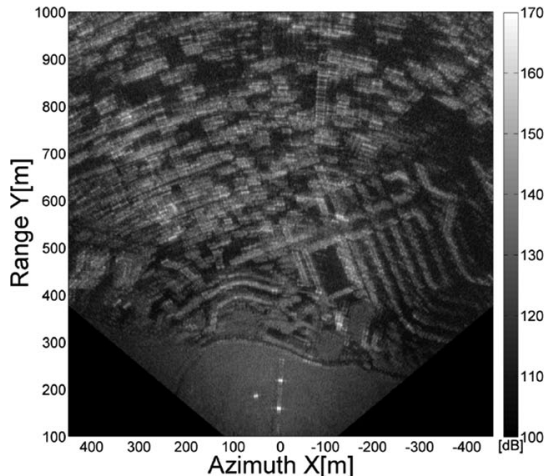

(b)

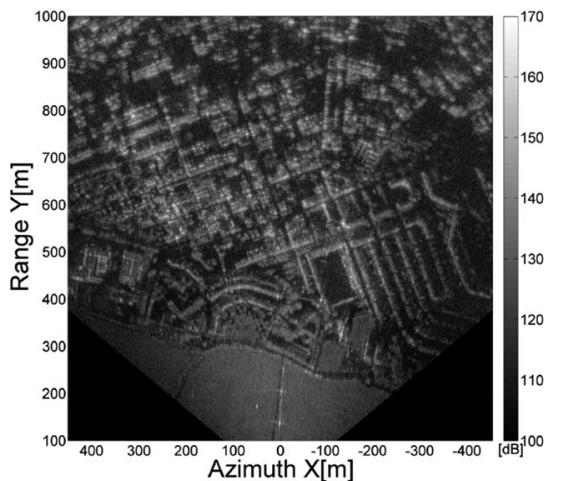

(e)

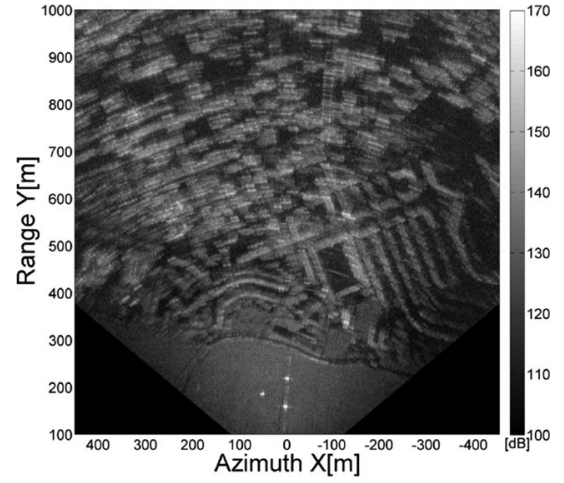

(c)

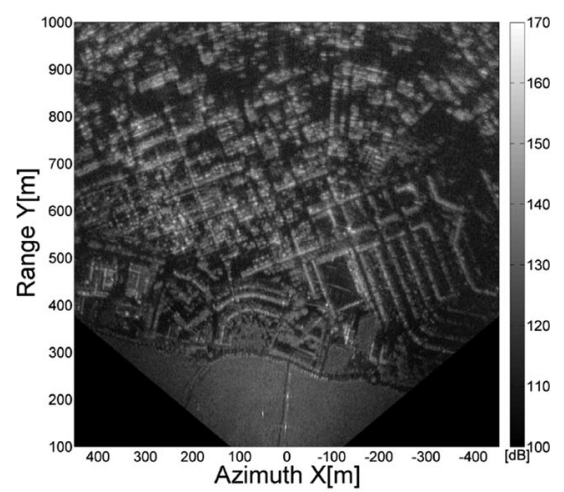

(f)

Fig. 7. SLC images in Cartesian coordinates for different aperture lengths before autofocus: (a) $13.4 \mathrm{~m}$, (b) $40.9 \mathrm{~m}$, and (c) $55.6 \mathrm{~m}$. Different aperture lengths after autofocus: (d) $13.4 \mathrm{~m}$, (e) $40.9 \mathrm{~m}$, and (f) $55.6 \mathrm{~m}$. Flight conditions: $150 \mathrm{~m}$ height, $v_{\mathrm{UAV}}=8.9 \mathrm{~m} / \mathrm{s}, \mathrm{VV}$ Pol.

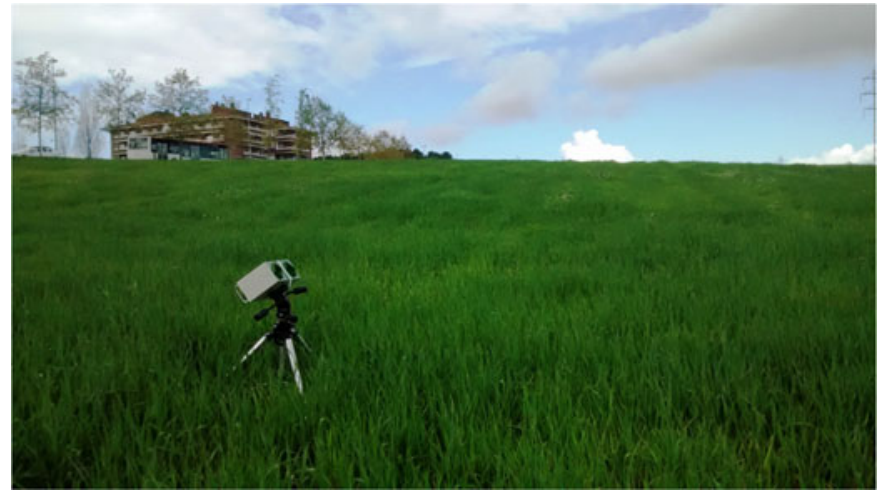

Fig. 8. PARC location in the agricultural field of the scenario.
The relative distance $R_{\text {PARC }}(t)$ can be expressed by the quadratic function as

$$
\begin{aligned}
R_{\mathrm{PARC}}(t)= & \left(\left(x_{\mathrm{PARC}}-x_{a}(t)-u_{x}(t)\right)^{2}+\right. \\
& \left.+\left(y_{\mathrm{PARC}}-u_{y}(t)\right)^{2}+\left(z_{\mathrm{PARC}}-u_{z}(t)\right)^{2}\right)^{1 / 2}
\end{aligned}
$$

where $x_{\text {PARC }}, y_{\text {PARC }}$, and $z_{\text {PARC }}$ correspond to the ideal position of the PARC in the $x-, y$-, and $z$-axis respectively, $x_{a}(t)$ is the azimuth antenna position during the slow time, and $u_{x}(t)$, $u_{y}(t)$, and $u_{z}(t)$ are the positioning errors of the platform in 291 the three axes with respect to the nominal flight path. Having 292 three PARCS, a system of three nonlinear equations and the three unknowns can be solved for every azimuth position of the platform. Thus, the deviations of the platform $u_{x}(t), u_{y}(t)$, and $u_{z}(t)$ can be derived to refocus the image taking these variations into account. The values obtained for the deviations of the platform must be filtered to avoid undesired effects of blurring in the compensated image. This is necessary because the retrieved parameters can present some noise that could be introduced by irregularities in the phase or by inaccuracies when solving the equations. Because of that, they must be filtered to prevent SLC images from being degraded when introducing the coefficients in the BPA. In our case, to perform the filtering, a moving average low-pass filter is used. Fig. 9 shows the retrieved information from the PARCs phase and the computed parameters to perform the autofocus.

Fig. 7(d)-(f) shows the three different SLC images obtained in Fig. 7(a)-(c) after applying the autofocus algorithm. The correction parameters $u_{x}(t), u_{y}(t)$, and $u_{z}(t)$ obtained in Fig. 9 are introduced in the focusing process to generate the images. Comparing Fig. 7(c) and (f), it is clearly visible that after applying the autofocus algorithm, the images do not show the azimuth defocusing for large apertures. Moreover, the fact of processing larger apertures implies a higher transmitted energy, i.e., higher SCR for deterministic targets, and an improvement in the quality 


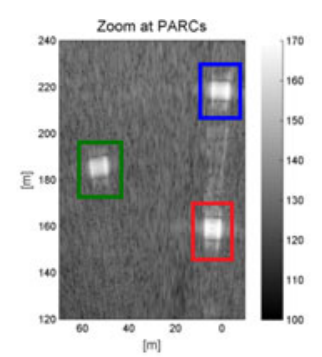

(a)

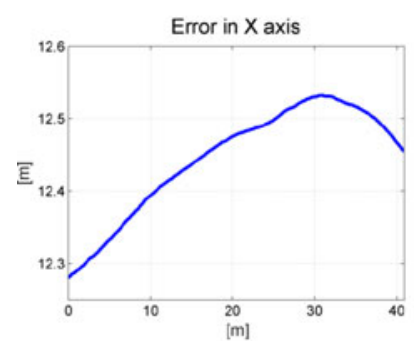

(e)

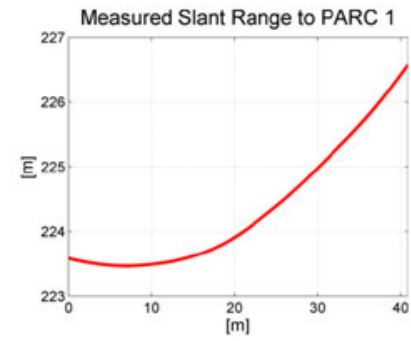

(b)

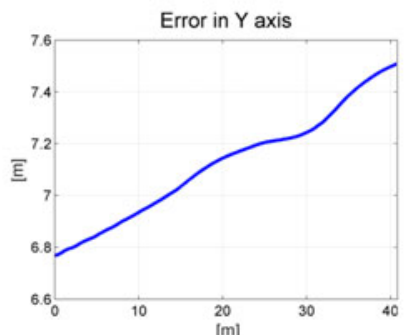

(f)

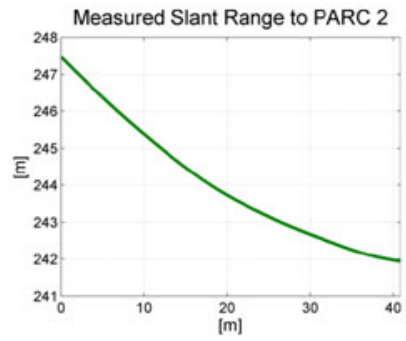

(c)

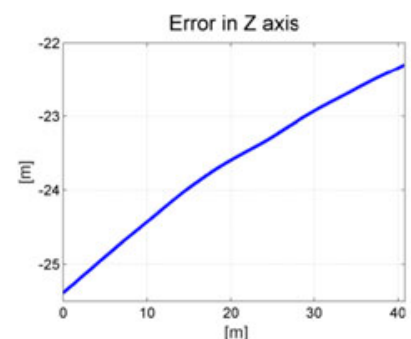

(g)

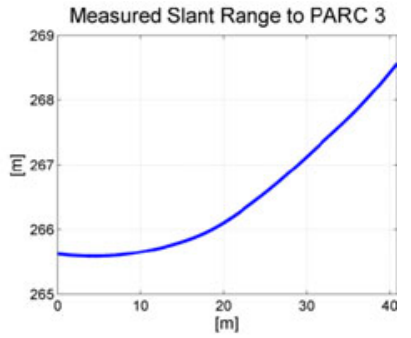

(d)

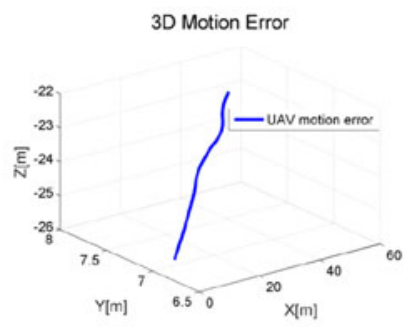

(h)

Fig. 9. Autofocus parameters for aperture of approximately $40.9 \mathrm{~m}$ and flight conditions: $150 \mathrm{~m}$ height, $v_{\mathrm{UAV}}=8.9 \mathrm{~m} / \mathrm{s}$, VV Pol. (a) SLC image in Cartesian coordinates with zoom to PARCs. Relative measured slant range to (b) PARC 1, (c) PARC 2, and (d) PARC 3 . Filtered deviation: (e) $u_{x}(t)$, (f) $u_{y}(t)$, and (g) $u_{z}(t)$. (h) Three-dimensional representation of the platform deviations.

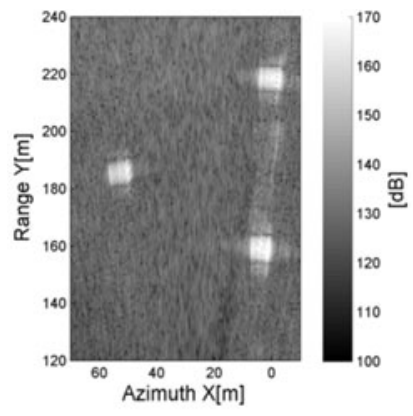

(a)

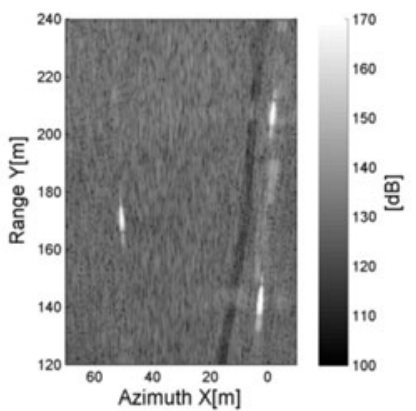

(b)

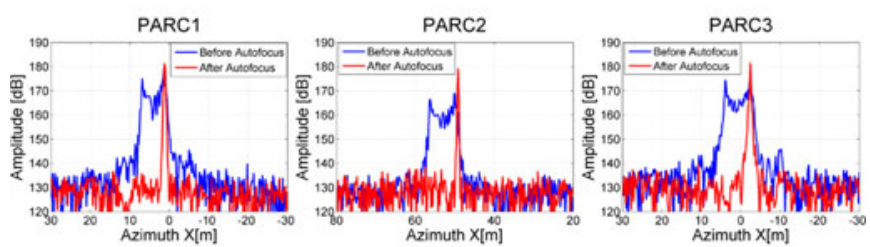

(c)

Fig. 10. Aperture length of $40.9 \mathrm{~m}$. Zoom to PARCs in SLC image (a) before and (b) after application of the autofocus algorithm. (c) Cut in azimuth of the PARCs before and after application of the autofocus algorithm. of the SLC images. The improvement in the azimuth resolution after applying the autofocus algorithm can be clearly seen in Fig. 10, where a zoom to the area containing the PARCs in the SLC images is shown. Even the track in the scenario is well defined in the image of Fig. 10(b), while in Fig. 10(a), it is not easily recognizable. The cut in azimuth of the three PARCs can be seen in Fig. 10(c). The response of the three PSs is clearly spread before applying the autofocus algorithm and gets better focused after applying it. Thus, the redistribution of the energy
TABLE II

AZIMUTH RESOLUTION

\begin{tabular}{lcc}
\hline \hline & $\Delta_{\mathrm{az}}$ Theoretical [m] & $\Delta_{\mathrm{az}}$ Measured [m] \\
\hline PARC 1 & 0.14 & 0.39 \\
PARC 2 & 0.16 & 0.22 \\
PARC 3 & 0.16 & 0.20 \\
\hline
\end{tabular}

in the focusing process results in higher values of amplitude 326 for the peak associated with the PARC, in particular 2.31, 10.2, 327 and $6.08 \mathrm{~dB}$, for PARCs 1,2, and 3, respectively. The use of an 328 aperture length shorter than the dimensions of the illuminated 329 scenario implies that the azimuth resolution $\Delta_{a z}$ will depend 330 on the range distance $R$, the length of the synthetic aperture $L, \quad 331$ and the squint angle $\theta$ as

$$
\Delta_{\mathrm{az}}=\frac{\lambda R}{2 L \cos ^{2}(\theta)} .
$$

Table II summarizes the theoretical and measured resolutions 333 after the autofocus algorithm for the aperture of $40.9 \mathrm{~m}$. By 334 means of (3), the theoretical azimuth resolutions of PARCs 335 1,2 , and 3 can be calculated, being $0.14,0.16$, and $0.16 \mathrm{~m}, 336$ respectively. Moreover, the measured 3-dB azimuth resolutions 337 after applying the autofocus algorithm are $0.39,0.22$, and 0.20338 $\mathrm{m}$ for PARCs 1,2 , and 3, respectively. It has to be considered 339 that, in order to reduce the secondary lobes that may degrade the 340 quality of the focused image, a Hanning filter window has been 341 applied in the cross-range domain, degrading the main lobe. 342 Taking this issue into account, the measured azimuth resolutions 343 after applying the autofocus algorithm are quite close to the 344 theoretical ones, although the autofocus algorithm could still be 345 improved. 


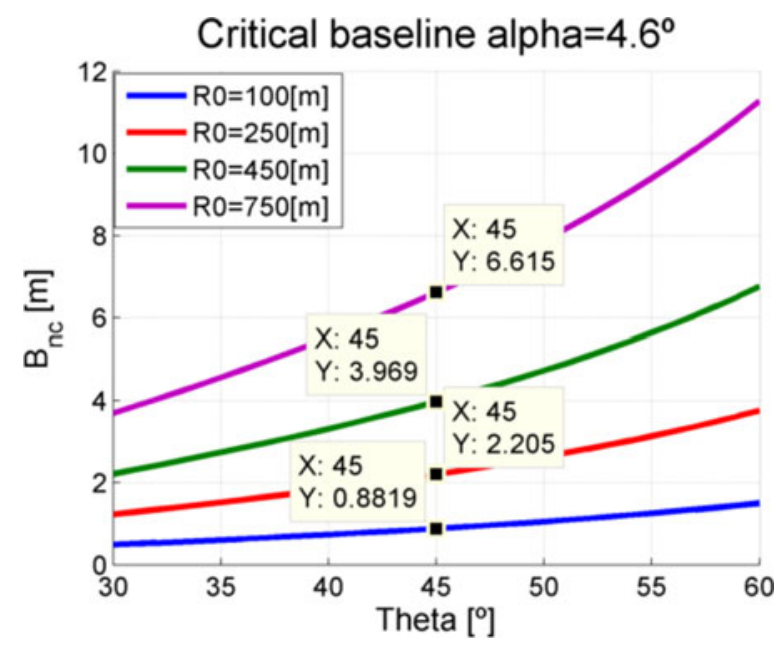

Fig. 11. Critical baseline as a function of the off-nadir angle for different $r_{0}$.

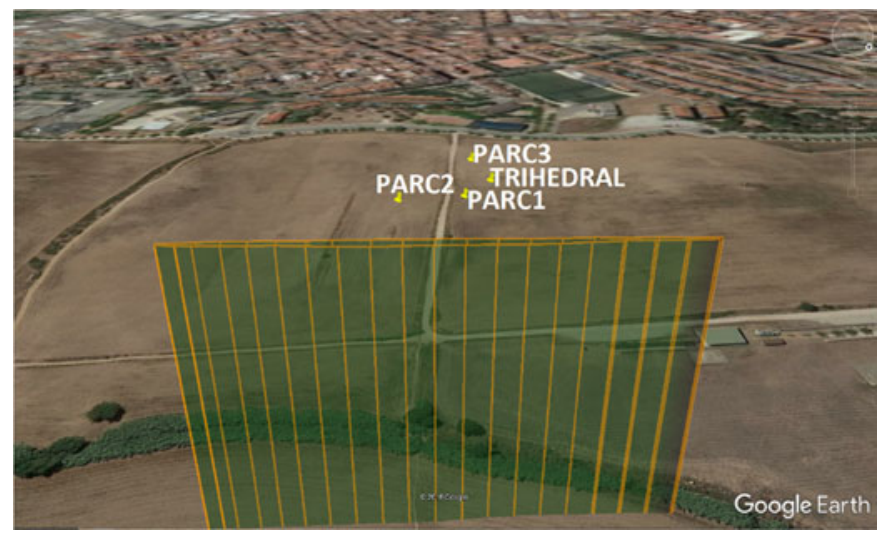

Fig. 12. Image of the scenario taken from Google Earth with the flight path information and the different calibrators.

The capability of the ARBRES-X SAR sensor integrated in the UAV MP to obtain SLC images has been demonstrated in this section.

\section{Innovative Flight Strategies in Airborne UAV MP Multidimensional SAR}

As has been pointed out previously, the flight capabilities of the UAV MP open new possibilities in airborne SAR observations. The present section exposes the repeat-pass interferometric and the fully polarimetric results obtained with the ARBRES-X SAR sensor integrated in the UAV MP platform. Furthermore, some results of measurements obtained performing vertical and circular flights are presented. The aim of this section is to assess the system performance and the SAR capabilities of the UAV MPs.

1) UAV MP SAR Repeat-Pass Interferometry: SAR interferometry exploits the phase differences between two sets of complex SAR data to derive, for example, topographic information. Differential SAR interferometry is a particular configuration, in which the interferometric data are obtained in different passes separated by a short baseline for deformation measurements

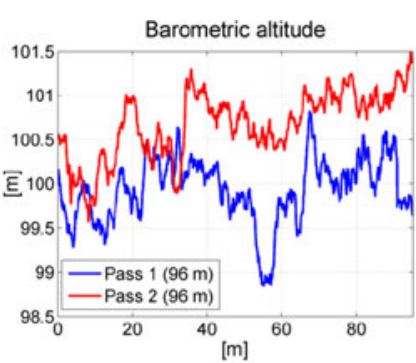

(a)

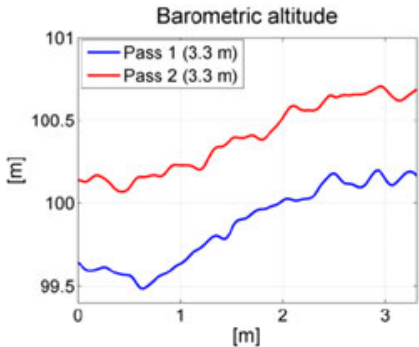

(c)

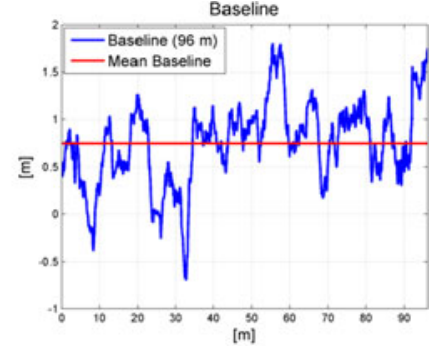

(b)

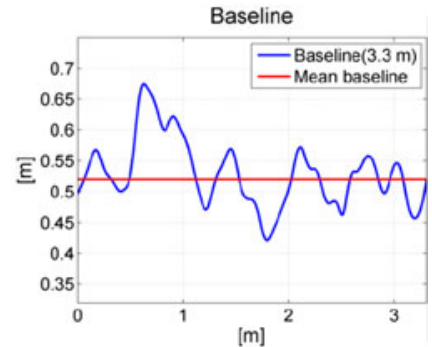

(d)
Fig. 13. Barometric altitude (a) and baseline (b) of the two passes of an aperture of approximately $96 \mathrm{~m}$ and (c) and (d) of an aperture of approximately $3.3 \mathrm{~m}$.

[11]. The antenna position that defines the baseline during the two passes is of great importance to have a good accuracy in the interferometric process. In the UAV MP, the baseline depends on the precision in positioning to perform the required flight paths and the undesired motion errors. Having a good control of the trajectory, together with the minimization of the instabilities of the platform, is mandatory to obtain interferometric results. According to [12], the total baseline decorrelation is reached in correspondence with the critical baseline

$$
\left|B_{n c}\right|=\left|\frac{\lambda r_{0} \tan (\theta-\alpha)}{2 \rho_{s}}\right|
$$

where $\lambda$ is the wavelength of the system, $r_{0}$ is the sensor to 376 target distance, $\theta$ is the off-nadir angle, $\alpha$ is the local terrain 377 slope (range), and $\rho_{s}$ is the slant range resolution. Fig. 11378 shows the evolution of the critical baseline as a function of $\theta$ for 379 different $r_{0}$.

In our particular case, the off-nadir angle of the ARBRES$\mathrm{X}$ SAR system integrated in the UAV MP is approximately $45^{\circ}$ and the local terrain slope of the illuminated scenario is approximately $4.6^{\circ}$ with an inclination of the $8 \%$ up to $300 \mathrm{~m}$. In Fig. 11, it can be seen that, for $r_{0}=100 \mathrm{~m}$ and $r_{0}=750 \mathrm{~m}$, the critical baseline is 0.88 and $6.6 \mathrm{~m}$, respectively.

Taking advantage of the possibility offered by the UAV MP to perform preprogrammed routes, repeat-pass interferometry strategies can be assessed. Fig. 12 shows an aerial view of the scenario with the flight path information, where two different passes of approximately $96 \mathrm{~m}$ in the stripmap configuration can be seen. The UAV MP was programmed to fly at $100 \mathrm{~m}$ with an interferometric baseline between passes of $0.8 \mathrm{~m}$, which is under the critical baseline calculated by (4) for $r_{0}=100 \mathrm{~m}$.
78 


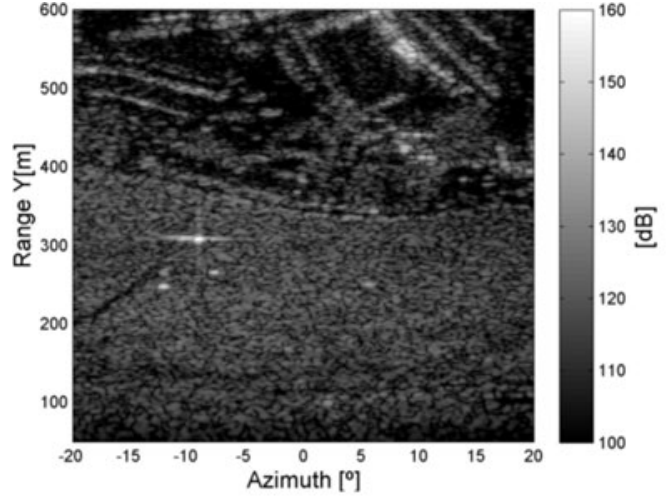

(a)

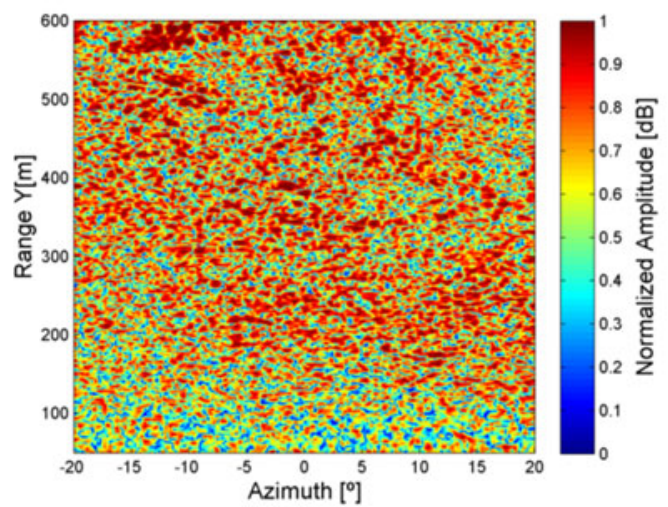

(c)

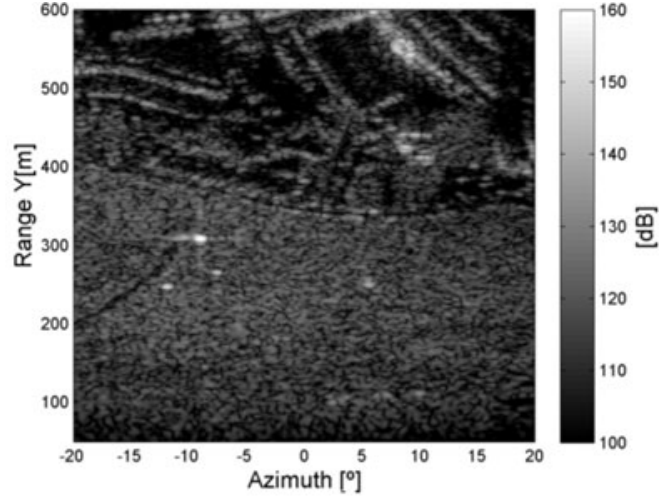

(b)

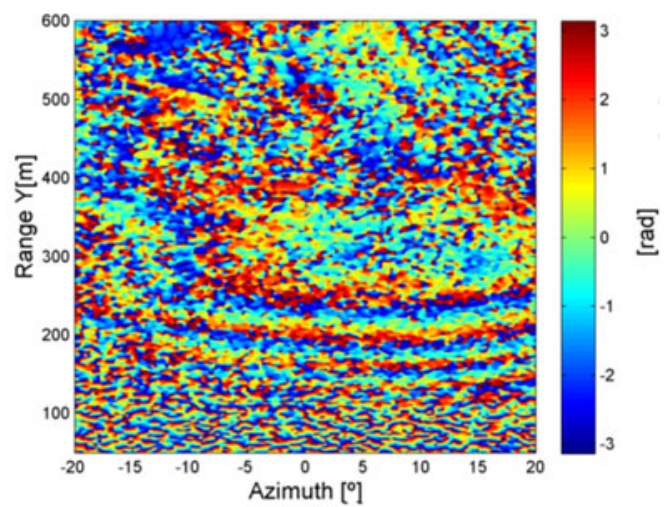

(d)

Fig. 14. Polar coordinates. (a) SLC pass 1. (b) SLC pass 2. (c) Coherence multilook $4 \mathrm{~m} \times 0.4^{\circ}$. (d) Interferogram multilook $4 \mathrm{~m} \times 0.4^{\circ}$. Flight conditions: 100 -m height, $v_{\mathrm{UAV}}=8.7 \mathrm{~m} / \mathrm{s}, 0.52-\mathrm{m}$ mean baseline, VV Pol.

Three PARCs and a trihedral were placed in the agricultural field to be used as references.

Fig. 13(a) and (b) shows the evolution of the barometric altitude and the interferometric baseline of the UAV MP, respectively, for the complete aperture of $96 \mathrm{~m}$. The mean baseline of the aperture is $0.74 \mathrm{~m}$, which is under the critical baseline. As it is clearly visible, the height of the platform was not constant during the acquisition time, which introduces a lot of focusing errors in the processing. Because of that, shorter apertures have to be processed to minimize the impact of the flight deviations and instabilities in the retrieved images, such as phase errors. The autofocus algorithm cannot be applied yet to compensate this motion errors in long apertures. This is because the correction is not as precise as needed in terms of the wavelength. Fig. 13(c) shows the barometric altitude for an aperture of approximately $3.3 \mathrm{~m}$, where the measurements are less affected by the instabilities of the UAV MP during the flight path. Furthermore, the selected part of the aperture for the two passes has a similar ascending behavior. Finally, Fig. 13(d) shows the interferometric baseline evolution for this shorter aperture, where the largest difference between the maximum and the minimum is $0.25 \mathrm{~m}$ and the mean baseline is $0.52 \mathrm{~m}$. At this point, it is worth mentioning that the theoretical accuracy of the navigation system in the positioning of the platform is $\pm 0.5 \mathrm{~m}$ in vertical and $\pm 1.5 \mathrm{~m}$ in horizontal. Furthermore, the velocity accuracy of the
UAV MP is $0.1 \mathrm{~m} / \mathrm{s}$, which is determined by the GPS. Moreover, 420 the system incorporates a barometer that has a vertical accuracy 421 of nearly $25 \mathrm{~cm}$. Despite these values, the parameters retrieved 422 from the IMU of the platform do not have enough accuracy to 423 quantify the precision of the flights. This can be observed, for 424 instance, in the data shown in Fig. 13, where the response of the 425 IMU represents unreal dynamics of the platform. An example 426 can be seen in the barometric altitude shown in Fig. 13(a), where 427 we can notice impossible variations in the height of the platform 428 of $1.25 \mathrm{~m}$ in $0.15 \mathrm{~s}$.

Fig. 14(a) and (b) shows the SLC images obtained with the 430 two different passes. The coherence and the retrieved interfer- 431 ometric phase are shown in Fig. 14(c) and (d), respectively. In 432 this case, the velocity during the short aperture has been as- 433 sumed constant, which is a feasible approximation, since the 434 acquisition time of $0.3 \mathrm{~s}$ is too short to allow great velocity 435 deviations.

As can be seen in Fig. 13, the motion errors of the platform in 437 each pass are independent during the interferogram generation. 438 In this case, differently from single-pass systems, the baseline 439 has a time-varying error that may cause significant phase errors 440 in range and azimuth direction. Nevertheless, Fig. 14 shows that, 441 despite having a nonconstant baseline during the selected part 442 of the aperture, the first interferogram with a UAV MP has been 443 obtained. However, it has to be admitted that it is extremely 444 


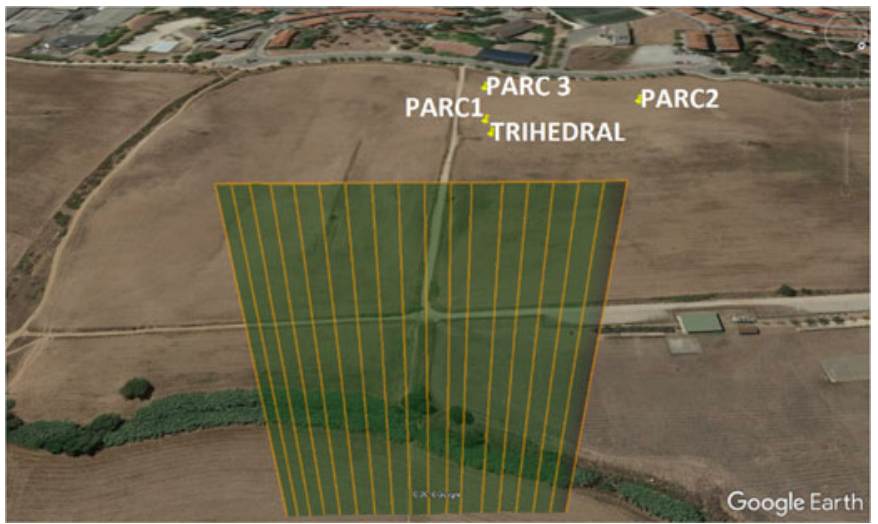

Fig. 15. Disposition of the calibrators in the scenario and flight path performed by the UAV MP. Image from Google Earth.

difficult to obtain interferometric maps, since perfectly parallel and aligned passes are difficult to achieve with the UAV MP. This is due to the lack of the required accuracy in the positioning of the platform and its inherent motion errors.

As the accuracy of the GPS and the IMU is not sufficient to retrieve the flight deviations that permit to let us know the correct baseline during the acquisition time, future steps include devoting some effort on the estimation and correction of timevarying baseline errors from the interferometric SAR data to improve the results, applying methods such as the one proposed in [13]. Moreover, a better navigation system operating with RTK GPS together with an IMU should be used, which would permit an accuracy of few centimeters both in vertical and horizontal. This way, larger apertures could be processed, and better interferometric results could be achieved.

2) Fully Polarimetric UAV MP SAR Measurements: To evaluate the fully polarimetric capabilities of the ARBRES-X SAR sensor, some measurements have been performed in the RACBSA airfield. To analyze the results, it has to be taken into account that, as said in Section II, four patch antennas have been used in the system. They have been selected for their simplicity, low weight, low size, and easy integration into the system, but they usually offer low levels of crosstalk. Despite this difficulty, the cross-polarization discrimination factor of the patch antennas is approximately $-22 \mathrm{~dB}$ [4]. Furthermore, the isolation between the two receiving channels is approximately $-30 \mathrm{~dB}$. Considering this, the first preliminary results presented in this section are not polarimetrically calibrated, but, as will be shown, the theoretical and measured results are consistent. In any case, the required calibration will not alter excessively the results.

Fig. 15 shows the flight path performed by the UAV MP and the disposition of different calibrators in the scenario. To assess the different polarizations, three PARCs and a trihedral corner reflector have been placed in the agricultural field of the area. Fig. 16(a) and (b) shows the photograph of PARC 1 and PARC 2, respectively. Fig. 16(c) shows PARC 3, which has been rotated $45^{\circ}$. Finally, the trihedral corner reflector is shown in Fig. 16(d).

The theoretical scattering matrices of the different elements are shown in (5), and the theoretical polarization signatures are shown in Fig. 17. The trihedral corner reflector is expected to

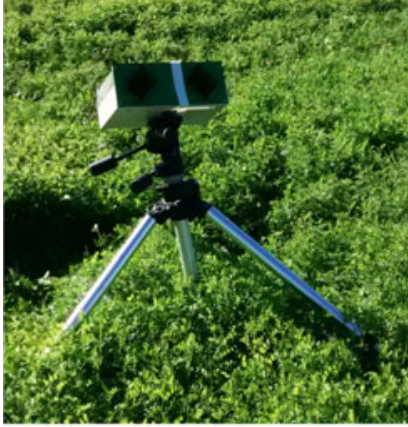

(a)

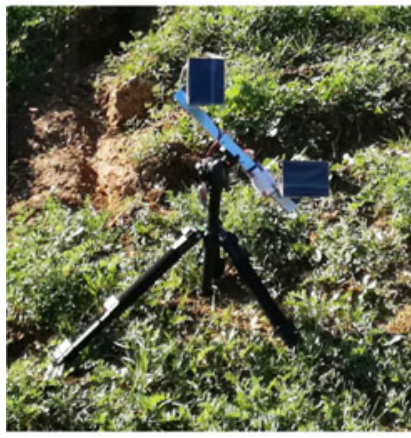

(c)

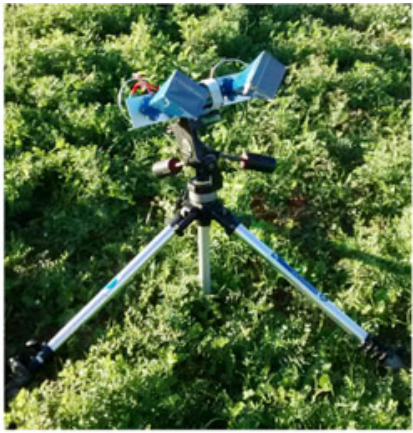

(b)

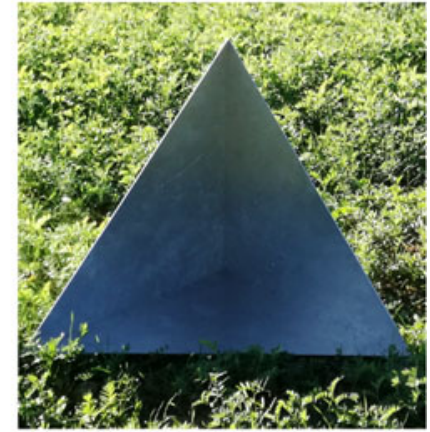

(d)
Fig. 16. Calibrators placed in the scenario. (a) PARC 1. (b) PARC 2 (c) PARC 3. (d) Trihedral corner reflector.

have identical horizontal and vertical backscattering sections, $\mathrm{HH}=\mathrm{VV}$, while no cross-polarization components are generated, $\mathrm{HV}=\mathrm{VH}=0$. PARC 1 and PARC 2 are expected to have cross-polarization and copolarization components, and, finally, PARC 3 is expected to have only the cross-polarization component $\mathrm{HV}$

$$
\begin{aligned}
S & =\left(\begin{array}{c}
S_{\mathrm{HH}} S_{\mathrm{HV}} \\
S_{\mathrm{VH}} S_{\mathrm{VV}}
\end{array}\right) \\
S_{P 1-2} & =\left(\begin{array}{ll}
1 & 1 \\
1 & 1
\end{array}\right), \quad S_{P 3}=\left(\begin{array}{ll}
0 & 1 \\
0 & 0
\end{array}\right), S_{T R I}=\left(\begin{array}{ll}
1 & 0 \\
0 & 1
\end{array}\right) .
\end{aligned}
$$

Fig. 18 shows the SLC images of the four polarizations $\mathrm{HH}$, $\mathrm{HV}, \mathrm{VH}$, and VV. As expected, PARC 1 and PARC 2 can be perfectly seen in all the polarizations, while the cross-polar polarization $\mathrm{HV}$ is the most important component of PARC 3. Besides, the most important components of the measured trihedral are $\mathrm{HH}$ and VV polarizations.

To evaluate the response of the calibrators to the different polarizations, the measured radar cross section (RCS) is shown in Fig. 19, and the retrieved scattering matrix can be seen in (6), which is derived from the measured RCS. Besides, the measured polarimetric signature of the calibrators obtained from 
Co-polarization signature of PARC1-2 Cross-polarization signature of PARC1-2
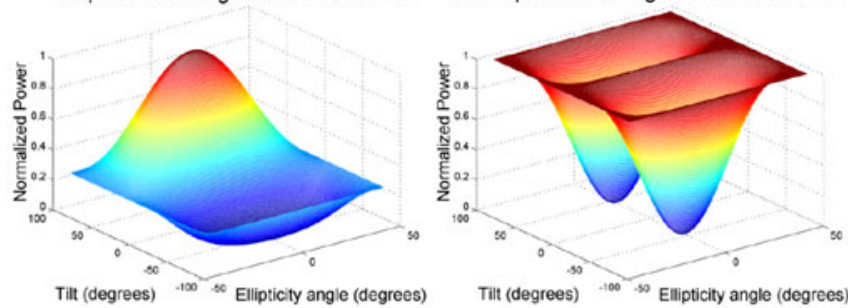

(a)

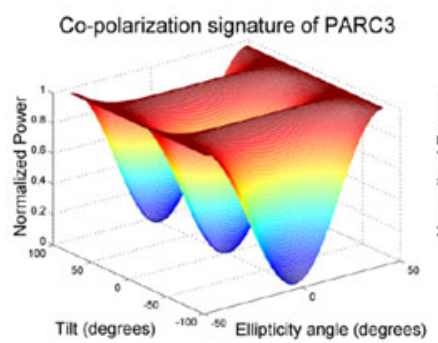

Cross-polarization signature of PARC3

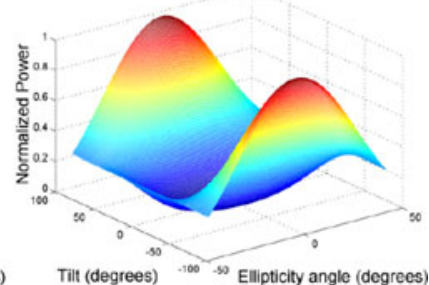

(b)

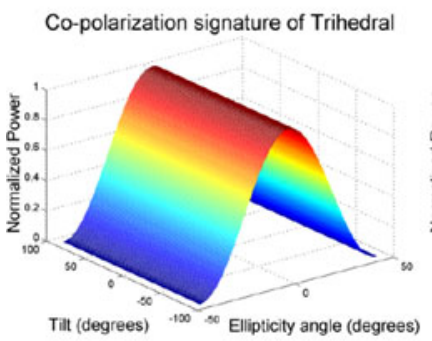

Cross-polarization signature of Trihedral

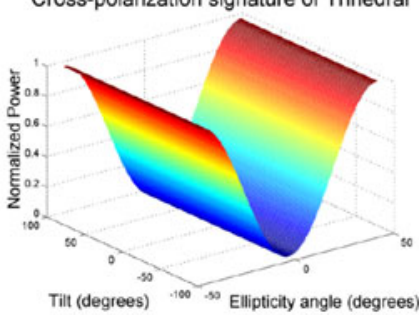

(c)

Fig. 17. Theoretical polarimetric signature of (a) PARC 1 and 2 and (b) PARC 3. (c) Trihedral corner reflector.

the scattering matrix is shown in Fig. 20

$$
\begin{array}{ll}
S_{P 1}=\left(\begin{array}{cc}
0.81 & 0.69 \\
1 & 0.78
\end{array}\right), & S_{P 2}=\left(\begin{array}{cc}
0.44 & 1 \\
0.23 & 0.49
\end{array}\right) \\
S_{P 3}=\left(\begin{array}{cc}
0.1 & 1 \\
0.02 & 0.15
\end{array}\right), & S_{\mathrm{TRI}}=\left(\begin{array}{cc}
1 & 0.08 \\
0.08 & 0.86
\end{array}\right) .
\end{array}
$$

Figs. 19 and 20 and (6) show that the polarimetric response of the different calibrators generally corresponds to the theoretical behavior, but with some variations. These differences are, in part, due to the difficulty to perfectly point the calibrators to the expected flight path. PARC 2, for example, has been placed in the scenario with an important squint angle, which makes it difficult to be well illuminated. Furthermore, the possible instabilities of the platform and the variation in the orientation of the antennas during the acquisition time can also degrade the polarimetric response.

Future steps include the polarimetric calibration of the system, which is not a trivial problem. In our system, the length of the synthetic aperture is short, and therefore, wide-beam antennas (approximately $60^{\circ}$ in our case) have to be used in order to obtain an image of suitable size. This implies a change of the polarization basis according to the illumination direction. This

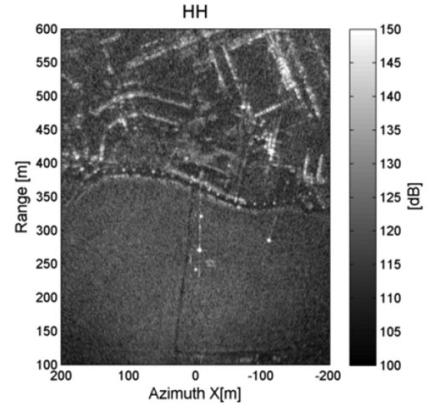

(a)

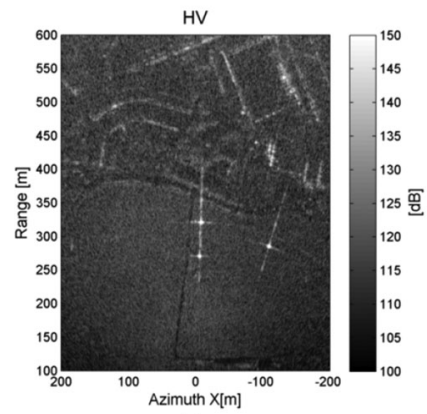

(c)

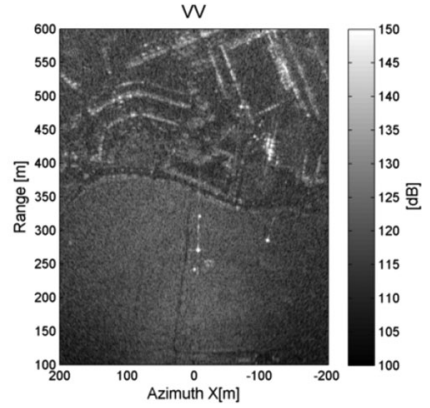

(b)

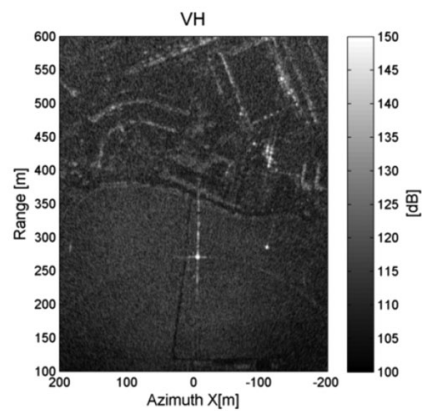

(d)
Fig. 18. SLC images of the different polarizations. (a) HH. (b) VV. (c) HV. (d) VH. Flight conditions: $150-\mathrm{m}$ height, $v_{\mathrm{UAV}}=8.9 \mathrm{~m} / \mathrm{s}$.

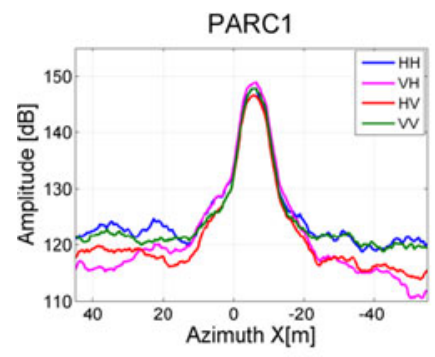

(a)

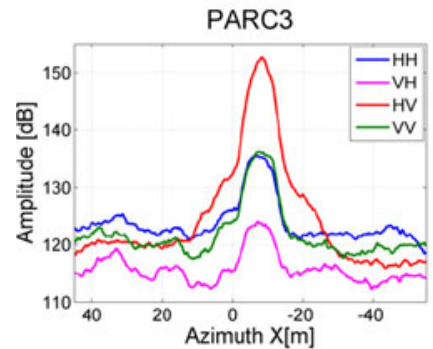

(c)

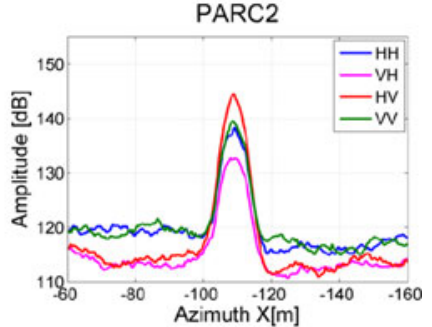

(b)

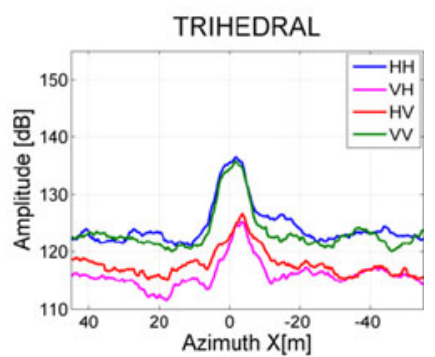

(d)
Fig. 19. Measured RCS of (a) PARC 1, (b) PARC 2, (c) PARC 3, and (d) trihedral corner reflector.

is not a problem in airborne and satellite SAR sensors, where 519 high-gain narrow beam antennas are used. As a consequence, 520 this factor must be taken into account to calibrate polarimetri- 521 cally the data.

3) Vertical Flight in UAV MP SAR: During the last years, 523 tomographic techniques for obtaining volume information of 524 


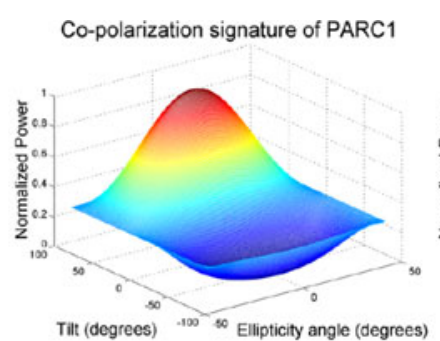

Cross-polarization signature of PARC1

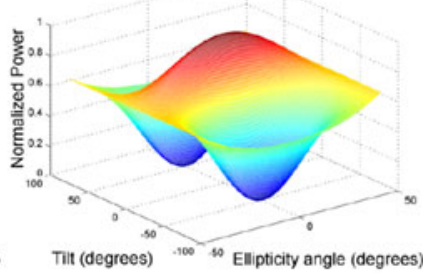

(a)

Co-polarization signature of PARC2

Cross-polarization signature of PARC2
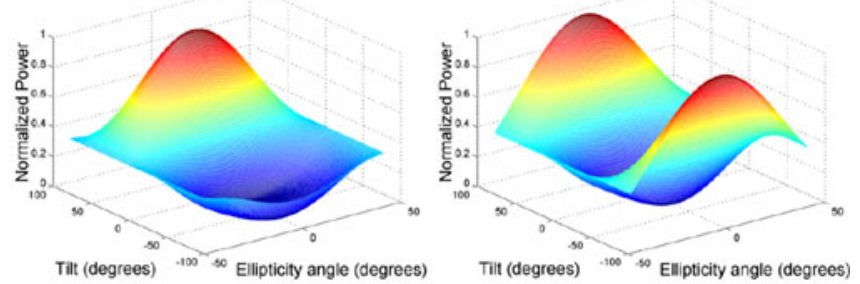

(b)

Co-polarization signature of PARC3

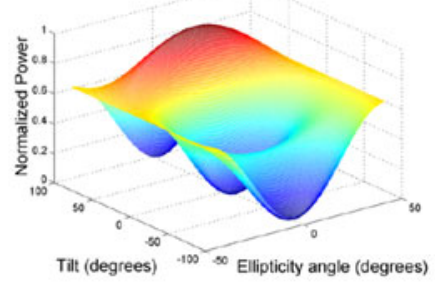

Cross-polarization signature of PARC3

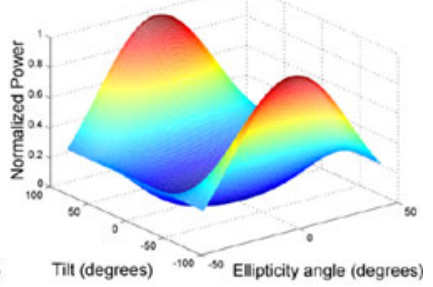

(c)

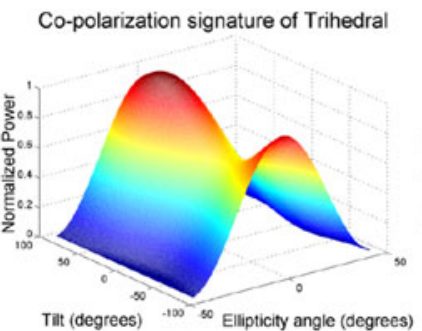

Cross-polarization signature of Trihedral

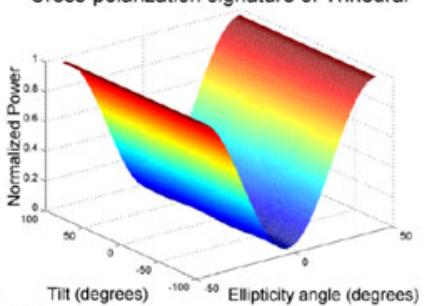

(d)

Fig. 20. Measured polarimetric signature of (a) PARC 1, (b) PARC 2, (c) PARC 3, and (d) trihedral corner reflector. scatterers have progressed considerably [14], [15]. The tomograms can be used, for instance, to monitor vegetated areas or to estimate ground topography. However, in order to retrieve the vertical scattering distribution, it is necessary to use multiple passes of the SAR sensor at different positions. In the airborne case, it is extremely difficult to obtain equally spaced baselines between the different passes. Besides, the flight paths can suffer deviations from the ideal straight trajectory in the $x-, y$-, and $z$-axis, which extremely complicates the processing of the data. In this context, the use of UAV MPs has several advantages that make it suitable to perform tomographic images. The possibility of this platform to perform vertical flights can be exploited to do vertical imaging in airborne SAR. With this strategy, the problems of the alignment and deviations of different flights might be

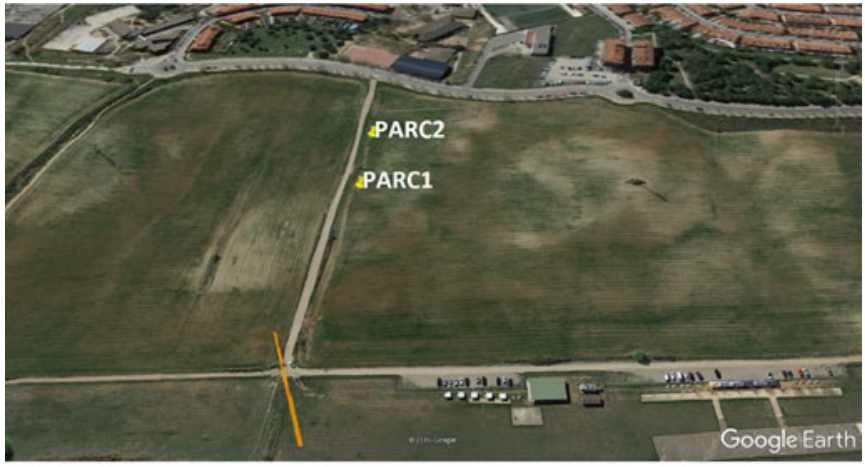

(a)

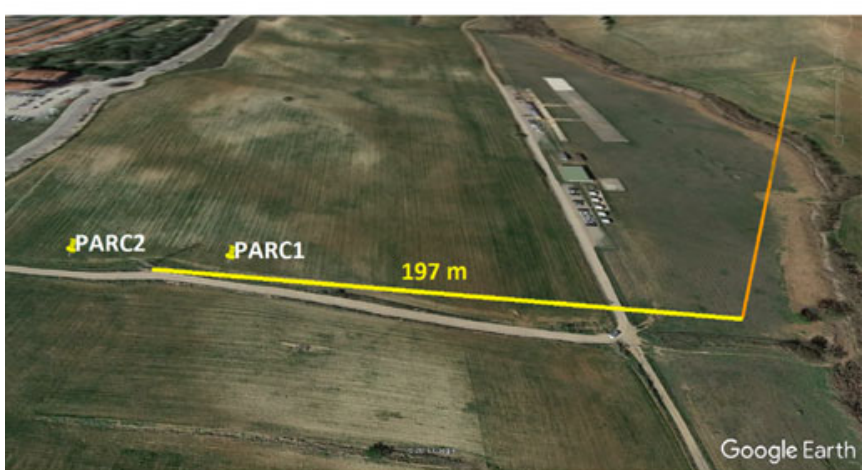

(b)

Fig. 21. Images of the scenario taken from Google Earth with the vertica flight path information. Ascending velocity of $2 \mathrm{~m} / \mathrm{s}$. (a) North-West orientation. (b) South-West orientation.

overcome. Fig. 21 shows two representations of a vertical flight path performed by the UAV MP taken from Google Earth. In our experience, the ascending vertical flight with the UAV MP is very stable and does not suffer from important deviations from the ideal flight path. Moreover, the ascending velocity of the platform is maintained almost constant, and the flight precision is equivalent to the horizontal stripmap acquisition mode.

In Fig. 21(b), a pylon is located at $197 \mathrm{~m}$ from the vertical flight trajectory. There are also two PARCs in the scenario at 173 and $226 \mathrm{~m}$. Moreover, the terrain of the scenario has an inclination of approximately $8 \%$ up to $300 \mathrm{~m}$, where the road separating the agricultural field from the city begins. Fig. 22(a) shows the SLC image of the scenario obtained with the ARBRES-X SAR sensor during a normal horizontal flight. The yellow line in the image corresponds to the vertical profile shown in Fig. 22(b), where the SLC obtained during the vertical flight is shown.

The pylon located at $197 \mathrm{~m}$ can be perfectly seen in Fig. 22(b) and also PARCs 1 and 2 at 173 and $226 \mathrm{~m}$, respectively. In the same way as in conventional SAR measurements, the resolution in elevation can be determined by (3). Table III summarizes the theoretical and measured resolutions for the 3-m aperture length. The theoretical azimuth resolutions for PARCs 1 and 2 are 1.48 and $1.96 \mathrm{~m}$, respectively, while the measured 3-dB azimuth resolutions are 1.93 and $2.08 \mathrm{~m}$. Again, considering the effect of the cross-range Hanning window, the measured resolutions are quite close to the theoretical ones. 


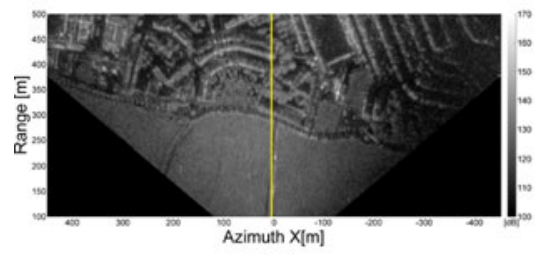

(a)

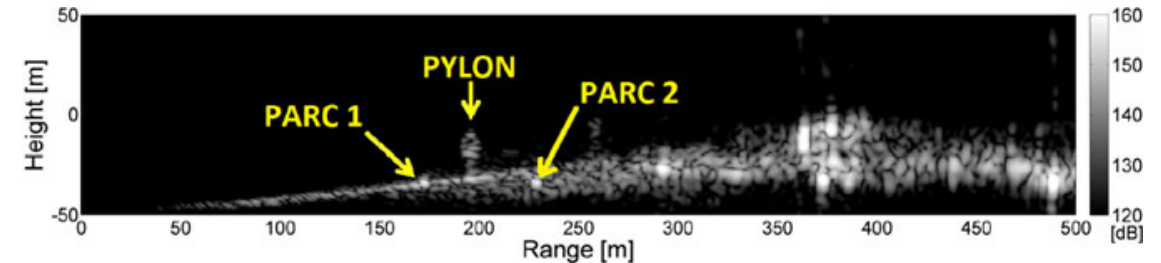

(b)

Fig. 22. (a) SLC image of the scenario during an horizontal aperture with flight conditions: $150-\mathrm{m}$ height, $v_{\mathrm{UAV}}=8.9 \mathrm{~m} / \mathrm{s}$, VV Pol. (b) SLC image of the scenario during the vertical flight with flight conditions: $v_{\mathrm{UAV}}=2 \mathrm{~m} / \mathrm{s}, \mathrm{VV}$ Pol, 3-m aperture length. The profile corresponds to the yellow line shown on the SLC image in (a).

TABLE III

AZIMUTH RESOLUTION OF THE VERTICAL FLIGHT

\begin{tabular}{lcc}
\hline \hline & $\Delta_{\mathrm{az}}$ Theoretical $[\mathrm{m}]$ & $\Delta_{\mathrm{az}}$ Measured $[\mathrm{m}]$ \\
\hline PARC 1 & 1.48 & 1.93 \\
PARC 2 & 1.96 & 2.08 \\
\hline
\end{tabular}

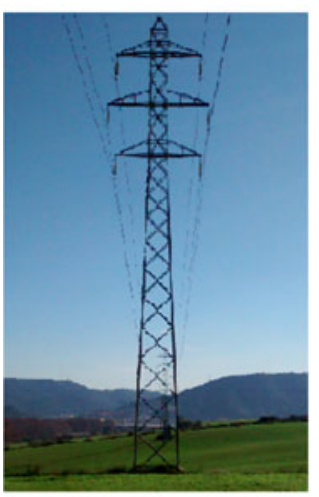

(a)

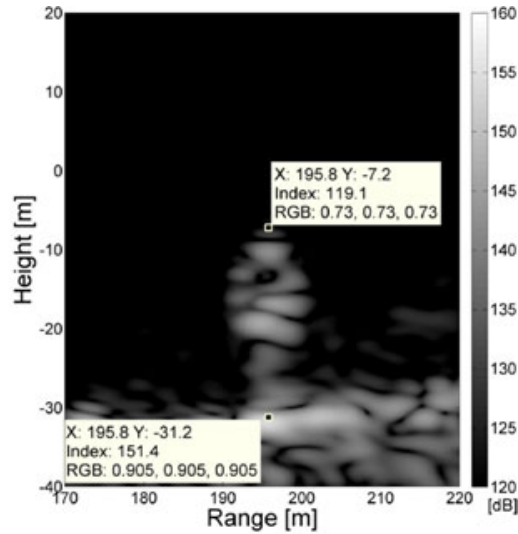

(b)

Fig. 23. (a) Photograph of the pylon in the scenario. (b) Zoom to the pylon in the vertical aperture of the scenario obtained with the ARBRES-X SAR sensor

Fig. 22(a) shows that the agricultural field of the scenario finishes approximately at $300 \mathrm{~m}$ and beyond this range distance starts the city. In Fig. 22(b), the contribution of all the scatterers covered by the antenna beamwidth corresponding to the urban area is collapsed at the same range. For that reason, there are a lot of bright pixels between 350 and $400 \mathrm{~m}$, which are the buildings of the city. The slope of the terrain derived from the vertical aperture is of approximately $8 \%$ up to $300 \mathrm{~m}$, what matches with the information obtained from topographic maps of the area. Fig. 23(a) shows a photograph of the pylon in the scenario, while Fig. 23(b) shows a zoom to the pylon observed in the vertical aperture of Fig. 22(b). The pylon has a height of $24 \mathrm{~m}$, which is comparable to the one obtained in the SLC image of Fig. 23(b), where the markers located at the base and the top of the pylon present a height difference of $24 \mathrm{~m}$.

In the way it is performed, the vertical image is affected by left-right ambiguity. Nevertheless, the goal of this experiment is to set the basis to vertical tomography, where this ambiguity is solved by considering a given number of vertical flights. In

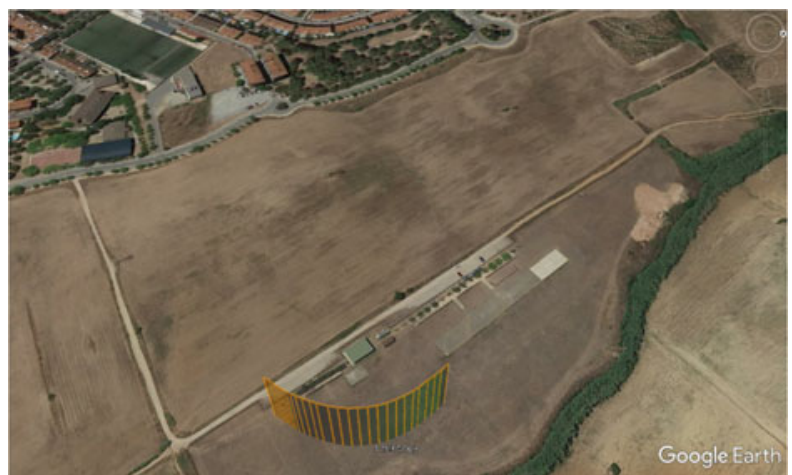

Fig. 24. Image of the scenario taken from Google Earth with the semicircular flight path information.

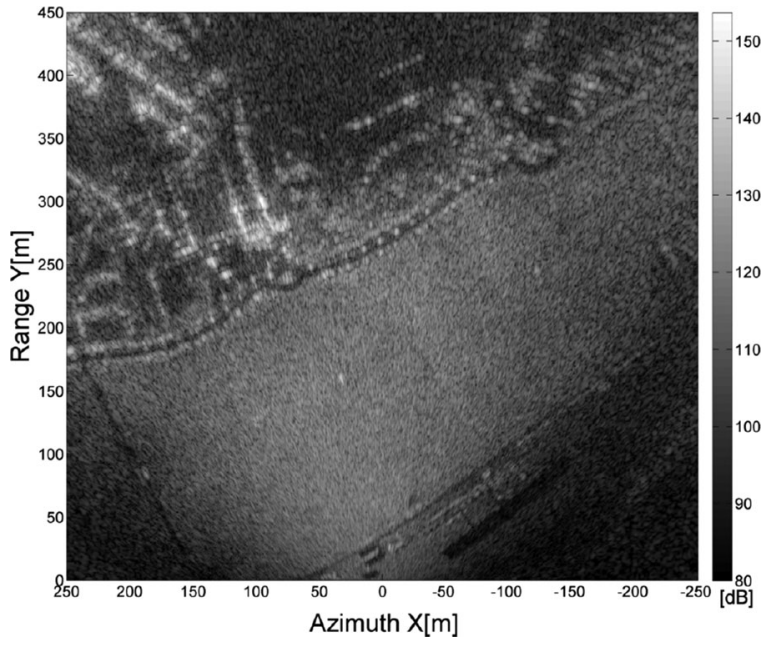

Fig. 25. SLC image of the scenario obtained with the ARBRES-X SAR sensor during the circular flight. Flight conditions: $50-\mathrm{m}$ height, $v_{\mathrm{UAV}}=6.2 \mathrm{~m} / \mathrm{s}$, VV Pol

addition, in the experiment, this ambiguity has a minimum effect in the imaged tower as it comes from a grass area.

4) Circular Flight in UAV MP SAR: The possibility of the UAV MP to perform circular flights can also be exploited to do tomographic imaging in the future, the so-called circular SAR [15]. In this context, by acquiring different circular trajectories over an area, several options are possible [16]. The purpose of this section is to demonstrate the feasibility of acquiring 591 SAR data with circular trajectories of the UAV MP. As a first 592 approximation to these type of flights, a partial segment of a 593 
circular flight is processed in the present paper, which allows us to envisage the possibility to perform complete circular flights in the future.

Fig. 24 shows the flight path information of the partial segment of a circular flight taken from Google Earth, and Fig. 25 shows the SLC image of the scenario obtained with the ARBRES-X SAR sensor during this trajectory. The aperture length is approximately $9.3 \mathrm{~m}$, and the platform height is approximately $50 \mathrm{~m}$.

Theoretically, the advantage of obtaining SAR images with circular flights is that they allow the maximum attainable resolution of a fraction of the wavelength [15]. In the SLC image obtained with the circular flight in Fig. 25, the area that theoretically has better resolution corresponds to the constructions in the airfield, such as the different buildings or the landing strip.

\section{CONCLUSION}

In the present paper, the SAR capabilities of UAV MPs have been evaluated. In this context, the integration of the ARBRESX SAR sensor into a small multicopter platform has been presented, opening new possibilities in airborne remote sensing. The feasibility of the system has been demonstrated by performing several measurement campaigns in the RACBSA airfield. Furthermore, the main limitations and technical challenges of obtaining SAR data with this type of platforms have been assessed along the present study.

The capability of the UAV MP to execute different types of trajectories has been exploited to evaluate the system performance and to obtain first preliminary results. It has been observed that the defocusing problem in the retrieved images is due to the deviation of the platform from the nominal trajectory and the flight instabilities. To overcome this undesired effect, it has been concluded that the use of a cost-effective small-size IMU is a nonviable option. This is because of the lack of accuracy of these systems, which has forced us to discard the use of a MoCo technique to focus the image. Alternatively, an autofocus algorithm has been applied to refocus the SLC images, improving its quality for large apertures.

Otherwise, the inherent instability of the platform and the insufficient positioning accuracy of the GPS system make it extremely difficult to obtain interferometric results. The autofocus algorithm is well suited to compensate amplitude images, but the problem is not yet efficiently resolved with the phase in long apertures. Because of that, the interferometric measurements have been processed using small apertures, where the flight deviations are less important. Nevertheless, the first SAR interferogram obtained with a repeat-pass flight performed with a UAV MP has been presented.

On the other hand, a vertical and a partial segment of a circular aperture have been successfully performed obtaining SLC images of the scenario, which envisages the capability of the UAV MP to perform tomographic images and complete circular apertures in the future. Regarding the vertical flight, it has been observed that the ascending velocity of the platform is very stable and the trajectory does not suffer from important deviations from the nominal track.
One of the limitations of ground-based SAR (GB-SAR) sensors is its dependence on the geometry of the scenario to illuminate the area under study. Sometimes, having the possibility to find the optimal position to place the GB-SAR sensor with the required orientation can be a problem. In the future, the use of UAV MPs can overcome this drawback allowing the monitoring of almost any site. In this sense, the use of SAR sensors integrated in UAV MPs can be considered as an interesting alternative between GB-SAR and airborne SAR sensors.

In conclusion, the UAV MP is a promising platform that opens new potentials for several applications, such as repeatpass interferometry or differential tomography imaging with the realization of almost arbitrary trajectories.

\section{REFERENCES}

[1] J.-S. Lee and E. Pottier, Polarimetric Radar Imaging: From Basics to Applications. Boca Raton, FL, USA: CRC Press, 2009, p. 440. [Online]. Available: http://www.crenetbase.com/doi/abs/10.1201/ 9781420054989.fmatt

[2] J. C. Curlander and R. N. McDonough, Synthetic Aperture RadarSystems and Signal Processing. Hoboken, NJ, USA: Wiley, 1991.

[3] R. F. Hanssen, Radar Interferometry - Data Interpretation and Error Analysis, vol. 2. New York, NY, USA: Springer, 2001. [Online]. Available: http://link.springer.com/10.1007/0-306-47633-9

[4] A. Aguasca, R. Acevo-Herrera, A. Broquetas, J. J. Mallorqui, and X. Fabregas, "ARBRES: Light-weight CW/FM SAR sensors for small UAVs," MDPI, vol. 13, no. 3, pp. 3204-3216, 2013.

[5] R. Acevo-Herrera, A. Aguasca, J. J. Mallorqui, and X. Fabregas, "Highcompacted FM-CW SAR for boarding on small UAVS," in Proc. Int Geosci. Remote Sens. Symp., 2009, vol. 2, pp. II-543-II-546.

[6] R. J. C. Middleton, "Dechirp-on-receive linearly frequency modulated radar as a matched-filter detector," IEEE Trans. Aerosp. Electron. Syst., vol. 48, no. 3, pp. 2716-2718, Jul. 2012.

[7] M. Soumekh, Synthetic Aperture Radar Signal Processing with MATLAB Algorithms. Hoboken, NJ, USA: Wiley, 1999. [Online]. Available: http://www.amazon.com/Synthetic-Aperture-SignalProcessing-Algorithms/dp/0471297062

[8] D. E. Wahl, P. H. Eichel, D. C. Ghiglia, and C. V. J. Jakowatz, "Phase gradient autofocus-A robust tool for high resolution SAR phase correction," IEEE Trans. Aerosp. Electron. Syst., vol. 30, no. 3, pp. 827-835, Jul. 1994.

[9] B. Fan, Z. Ding, W. Gao, and T. Long, "An improved motion compensation method for high resolution UAV SAR imaging," Sci. China Inf. Sci. vol. 57, no. C, pp. 1-13, 2014

[10] A. Alabort and J. J. Mallorqui, "Development of motion compensation algorithms for airborne UAV SAR," 2014

[11] P. Rosen et al., "Synthetic aperture radar interferometry," Proc. IEEE, vol. 88, no. 3, pp. 333-382, Mar. 2000. [Online]. Available: http:// ieeexplore.ieee.org/lpdocs/epic03/wrapper.htm?arnumber $=838084$

[12] F. Gatelli, A. M. Guarnieri, F. Parizzi, P. Pasquali, C. Prati, and F. Rocca, "The wavenumber shift in SAR interferometry," IEEE Trans. Geosci. Remote Sens., vol. 32, no. 4, pp. 855-865, Jul. 1994.

[13] A. Reigber, P. Prats, and J. J. Mallorqui, "Refined estimation of timevarying baseline errors in airborne SAR interferometry," in Proc. Int Geosci. Remote Sens. Symp., 2005, vol. 7, pp. 4799-4802.

[14] A. Reigber and A. Moreira, "First demonstration of airborne SAR tomography using multibaseline L-band data," IEEE Trans. Geosci. Remote Sens., vol. 38, no. 5 I, pp. 2142-2152, Sep. 2000.

[15] A. Moreira, P. Prats-iraola, M. Younis, G. Krieger, I. Hajnsek, and K. P. Papathanassiou, "A tutorial on synthetic aperture radar," IEEE Geosci. Remote Sens. Mag., vol. 1, no. 1, pp. 6-43, Mar. 2013. [Online]. Available: http://ieeexplore.ieee.org/lpdocs/epic03/wrapper.htm?arnumber= 6504845

[16] O. Ponce, P. Prats-Iraola, R. Scheiber, A. Reigber, A. Moreira, and E. Aguilera, "Polarimetric 3-D reconstruction from multicircular SAR at Pband," IEEE Geosci. Remote Sens. Lett., vol. 11, no. 4, pp. 803-807, Apr. 2014. 


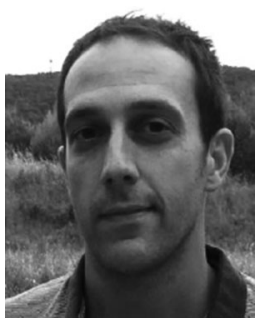

Marc Lort was born in Sabsadell, Spain, in 1986. He received the B.Sc. degree in telecommunication engineering from the Universitat Autònoma de Barcelona, Barcelona, Spain, in 2010. In 2013, he joined the Signal Theory and Communications Department, Universitat Politècnica de Catalunya (UPC), Barcelona, and received the M.Sc. degree in 2014. He is currently working toward the Ph.D. degree, focused on multidimensional synthetic aperture radar (SAR) for the Earth observation, with UPC.

His main research interests include the development and test of SAR systems integrated in unmanned aerial vehicles.

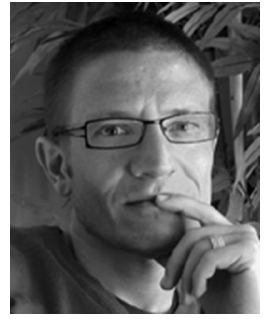

Carlos López-Martínez (S'97-M'04-SM'11) re- 744 ceived the M.Sc. degree in electrical engineering and 745 the Ph.D. degree from the Universitat Politècnica de 746 Catalunya, Barcelona, Spain, in 1999 and 2003, re- 747 spectively.

From October 2000 to March 2002, he was with the Frequency and Radar Systems Department, German Aerospace Center (DLR), Oberpfaffenhofen, Germany. From June 2003 to December 2005, he was with the Image and Remote Sensing GroupSAPHIR Team, Institute of Electronics and Telecommunications of Rennes (I.E.T.R. CNRS UMR 6164), Rennes, France. In January 2006, he joined as a Ramn-y-Cajal Researcher the Universitat Politècnica de Catalunya, Barcelona, Spain, where he is currently an Associate Professor in the area of remote sensing and microwave technology. He has organized different invited sessions in international conferences on radar and synthetic aperture radar (SAR) polarimetry. He has presented advanced courses and seminars on radar polarimetry to a wide range of organizations and events. He has authored or coauthored more than 100 articles in journals, books, and conference proceedings in the radar remote sensing and image analysis literature. His research interests include SAR and multidimensional SAR, radar polarimetry, physical parameter inversion, digital signal processing, estimation theory, and harmonic analysis.

Dr. López-Martínez is an Associate Editor of the IEEE JOURNAL OF SELECTED TOPICS IN ApPliEd EARTH OBSERVATIONS AND REMOTE SENSING. He served as a Guest Editor of the EURASIP Journal on Advances in Signal Processing. He received the Student Prize Paper Award at the EUSAR 2002 Conference and coauthored the paper awarded with the First Place Student Paper Award at the EUSAR 2012 Conference. He has also received the IEEE-GRSS 2013 GOLD Early Career Award.

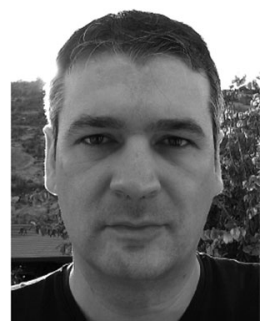

Tomás Martínez Marín received the Tech.Eng. 775 (B.S.) degree from the University of Alcalá, Alcalá 776 de Henares, Spain, in 1990, and the M.S. and Ph.D. 777 degrees in telecommunications engineering from the 778 Technical University of Madrid, Madrid, Spain, in 779 1995 and 1999, respectively .

He joined the University of Alcalá as an Assistant Professor in 1990. In 1997, he joined the European University of Madrid as an Assistant Professor. Since 2000, he has been with the Department of Physics, System Engineering and Signal Theory, University of Alicante, Alicante, Spain, where he is currently an Associate Professor. His research interests include reinforcement learning, intelligent vehicles, simultaneous localization and mapping, polarimetric and interferometric techniques, and synthetic aperture radar imaging algorithms. thored more than 40 papers on microwave synthetic aperture radar (SAR), radiometer systems, and microwave circuits. His research interests include the design and development of SAR and microwave radiometer systems for unmanned aerial vehicle platforms. UPC. His teaching activities involve radio-frequency and microwave circuits for communications and radio navigation systems. He has authored or coau-

Barcelona, Spain, in 1964. He received the M.Sc. and Ph.D. degrees in telecommunication engineering arcelona, in 1989 and 1993, respectively.

Since 1995, he has been an Associate Professor

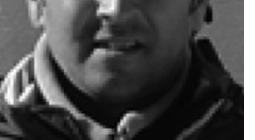


- Authors: When accessing and uploading your corrections at the Author Gateway, please note we cannot accept new source files as corrections for your paper. Do not send new Latex, Word, or PDF files, as we cannot simply "overwrite" your paper. Please submit your corrections as an annotated PDF or as clearly written list of corrections, with location in paper. You can also upload revised graphics to the Gateway.

- Authors: Please note that once you click "approve with no changes," the proofing process is now complete and your paper will be sent for final publication and printing. Once your paper is posted on Xplore, it is considered final and the article of record. No further changes will be allowed at this point so please ensure scrutiny of your final proof.

- Authors: Unless invited or otherwise informed, a mandatory Excessive Paper Length charge of \$200.00 per page (beginning with page 7 and beyond) is required for papers in excess of six (6) printed pages for JSTARS. If you have any questions regarding overlength page charges, need an invoice, or have any other billing questions, please contact reprints@ieee.org as they handle these billing requests.

Q1. Author: Please provide full bibliographic details in Ref. [10].

Q2. Author: Please provide the subjects in which the author Carlos López-Martínez received the Ph.D. degree.

Q3. Author: Please provide the subjects in which the author Tomás Martínez Marín received the Tech.Eng. (B.S.) degree. 


\title{
Initial Evaluation of SAR Capabilities in UAV Multicopter Platforms
}

\author{
Marc Lort, Albert Aguasca, Member, IEEE, Carlos López-Martínez, Senior Member, IEEE, \\ and Tomás Martínez Marín
}

\begin{abstract}
Airborne synthetic aperture radar (SAR) sensors have been commonly used during the last decades to monitor different phenomena in medium-scale areas of observation, such as object detection and characterization or topographic mapping. The use of unmanned aerial vehicles (UAVs) is a cost-effective solution that offers higher operational flexibility than airborne systems to monitor these types of scenarios. The Universitat Politècnica de Catalunya has developed the first fully polarimetric SAR system at X-band integrated into a small UAV multicopter platform (UAV MP). The sensor, called AiR-based remote sensing, has been integrated into the platform overcoming restrictions of weight, space, robustness, and power consumption. The present paper introduces the SAR capabilities of UAV MPs, which open new unexploited potentials to airborne SAR. To demonstrate the validity of the developed system, some measurement campaigns have been conducted in the outskirts of Barcelona, Spain.
\end{abstract}

Index Terms-Airborne synthetic aperture radar (SAR), unmanned aerial vehicle (UAV) multicopter, UAV SAR.

\section{INTRODUCTION}

$\mathbf{S}$ YNTHETIC aperture radar (SAR) sensors allow a flexible surface observation in innumerous applications areas such as agriculture, forestry, topography, earth-resource mapping, or environmental monitoring [1]-[3]. Unmanned aerial vehicles (UAVs) represent an alternative to be considered in airborne remote sensing in terms of cost effectiveness and operational flexibility. Nevertheless, these platforms have some limitations when used in SAR applications. One of the most important is the undesired deviations from the nominal flight trajectory that may cause defocusing, geometric distortions, or phase errors in the SAR images.

The purpose of this work is to validate the system performance and to evaluate the SAR capabilities in UAV multicopter

Manuscript received February 2, 2017; revised May 22, 2017 and July 17, 2017; accepted September 5, 2017. This work was supported by the Spanish Ministry of Science and Innovation (MICINN) under Project TIN201455413C21P and Project TIN201455413C22P, the Research Grant BES-2012-060089, and the Unidad de Excelencia Maria de Maeztu MDM2016-0600, which is financed by the Agencia Estatal de Investigación, Spain. (Corresponding author: Marc Lort.)

M. Lort, A. Aguasca, and C. López-Martínez are with the CommSensLab, Universitat Politècnica de Catalunya, 08034 Barcelona, Spain (e-mail: marc.lort@tsc.upc.edu; aguasca@tsc.upc.edu; carlos.lopez@tsc.upc.edu).

T. M. Marín is with the Department of Physics, System Engineering and Signal Theory, University of Alicante, E-03690 Alicante, Spain (e-mail: tomas.martinez@ua.es).

Color versions of one or more of the figures in this paper are available online at http://ieeexplore.ieee.org.

Digital Object Identifier 10.1109/JSTARS.2017.2752418

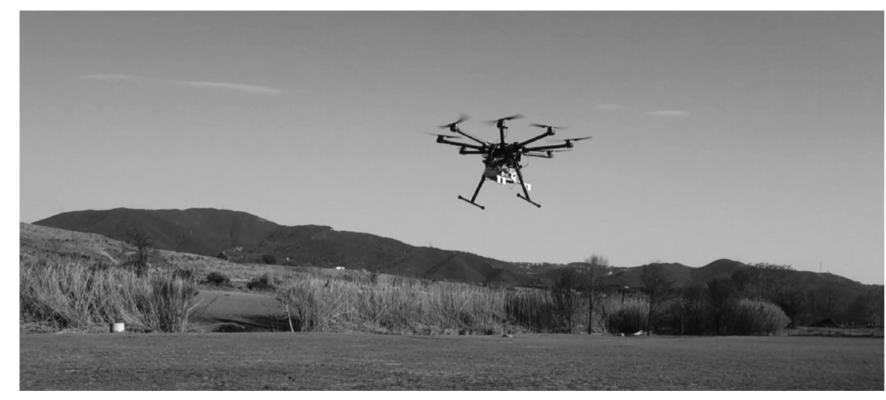

Fig. 1. Fully polarimetric ARBRES-X SAR integrated in the UAV MP.

platforms (UAV MP), such as repeat-pass interferometry, polarimetric measurements, and vertical or circular trajectories that can be used, for instance, for tomographic purposes. The use of this platform introduces new possibilities in airborne SAR observation due to its inherent flight capabilities and characteristics.

The present paper is organized as follows. Section II presents the system description, the architecture, and the setting parameters of the ARBRES-X SAR sensor. Furthermore, the UAV MP used to integrate the ARBRES-X SAR sensor is introduced, and its main advantages and disadvantages are presented. In Section III, the experimental results are assessed. The stability of the platform is evaluated as a function of the aperture length, and an autofocus algorithm is applied to improve the quality of the retrieved images. Moreover, different innovative flight strategies for airborne SAR observation are introduced, such as vertical flights, circular flights, or repeat-pass flights for interferometry. Finally, the main conclusions and major remarks are given in Section IV.

\section{UAV MP AND SAR SYSTEM DESCRIPTION}

In this section, the main components and characteristics of the integrated system are presented. The different elements are the ARBRES-X SAR sensor, the UAV MP, and the inertial measurement unit (IMU). Fig. 1 shows a picture of the complete system flying during a measurement campaign.

\section{A. UAV Multicopter Platform}

The Spreading Wings S1000 from DJI, designed for professional aerial photography and cinematography, has been chosen to integrate the ARBRES-X SAR sensor. UAV MPs have several advantages compared with a fixed-wing airplane UAV that 
makes them suitable to integrate a sensor to perform SAR measurements. The main advantage of the UAV MP is that it does not need a long landing strip. Due to its vertical lift capability, it only needs a small area, such as a forest clearing, to take off and to land, making it operational in almost any location. Besides, its capacity to hover and perform agile maneuvering makes this type of platforms well suited for many applications in airborne SAR observation. Some examples could be object detection or urban structure characterization, where precision flights and the ability to maintain the observation on a single target for extended periods of time may be required.

Another important advantage is that the UAV MP can perform 3-D flights due to its ability to move in almost any direction, opening a wide range of new possibilities in airborne SAR measurements. Vertical and circular flights can be combined, for example, to define new strategies to obtain 3-D measurements of structures. Vertical flights can be used to perform vertical apertures and also to think about high-resolution tomography. On the other hand, circular flights can be used, for instance, to obtain holographic/tomographic SAR images. Finally, some preprogrammed flights with autotrigger can be performed with the autopilot function of the UAV MP. It is important because it opens the possibility to predefine different strategies of flight, such as repeat-pass interferometry in airborne SAR, with a good control of the route and the measurement time. All these advantages make the UAV MP a versatile platform that gives a greater degree of operational flexibility with respect to the conventional UAVs.

Despite the important advantages of the UAV MP, some drawbacks have also to be pointed out. It is inherently unstable if compared with the fixed-wing UAVs, so its capability to perform constant-velocity straight linear trajectories is reduced. This is extremely important because errors in positioning and velocity of the platform during the acquisition time affect drastically to the measurements performed with the ARBRES-X SAR sensor. In this context, Fig. 2 is intended to provide a graphical description of the effects of having nonuniform trajectories during the realization of the measurements.

Fig. 2(a) shows the example of a geometry considering an ideal flight path and a flight with deviations in height from the nominal track. In Fig. 2(b) and (c), the focusing of a permanent scatterer (PS) has been simulated considering an ideal flight path and a flight path with deviations in height. The focused singlelook complex (SLC) image of the PS obtained with the nonideal flight presents an important defocusing in the azimuth direction due to the deviations of the platform from the nominal track. For this reason, these effects must be compensated for when focusing the images, taking into account that the deviations from the ideal track can be present in the three spatial coordinates $x$, $y$, and $z$.

Another disadvantage of the UAV MP is the short flight autonomy, with respect to the fixed-wing UAVs, which reduces the total area that can be covered. The flight time, which depends on the power consumption of the system, is related to the total weight of the platform. This imposes several restrictions in weight for the integration of the ARBRES-X SAR sensor and

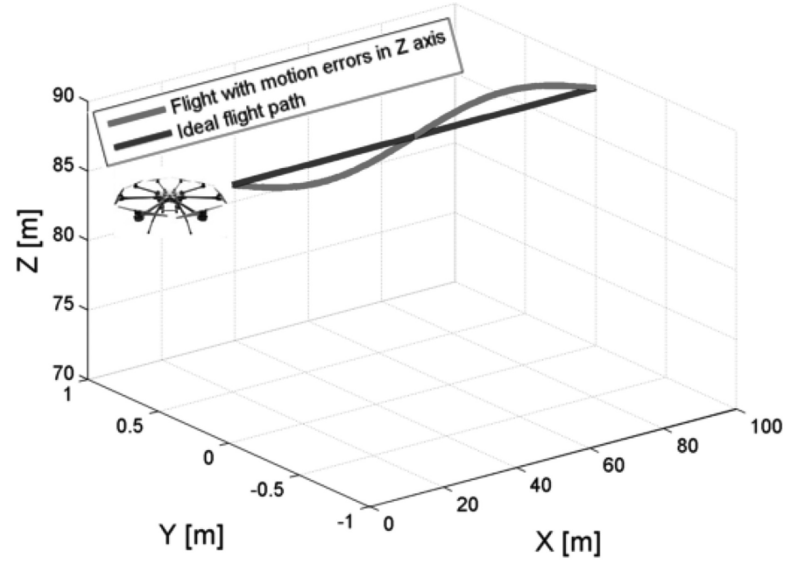

(a)

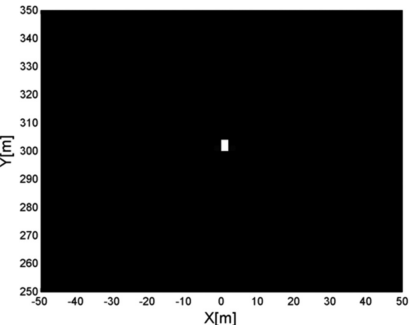

(b)

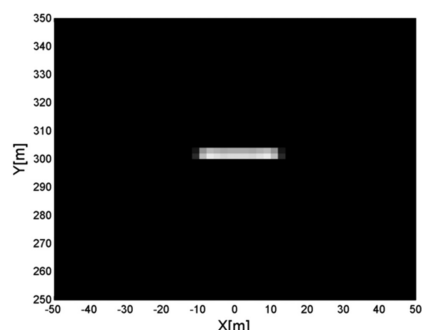

(c)
Fig. 2. (a) Representation of the geometry of a nonideal flight path with deviations in the $z$-axis. (b) SLC of a simulated target of an ideal flight path (c) SLC of a simulated target of a nonideal flight path with deviations in the $z$-axis.

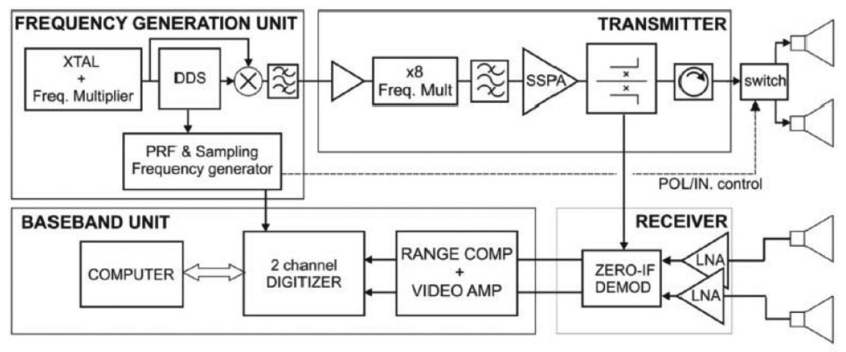

Fig. 3. ARBRES-X system architecture.

all the additional electronics, such as the trigger control or the IMU.

The whole system requires the integration of the ARBRES-X SAR sensor, the RF front-end bar, the IMU, the electronics of control, and the batteries into the UAV MP platform without exceeding the maximum takeoff weight. The SAR sensor, with a weight of approximately $2 \mathrm{~kg}$, has been placed in the lower part of the UAV MP together with the batteries of the platform. The total payload is around $6.5 \mathrm{~kg}$, and the whole integrated system weights around $10.5 \mathrm{~kg}$, slightly below the maximum takeoff weight of $11 \mathrm{~kg}$.

\section{B. ARBRES-X SAR Sensor}

The ARBRES-X SAR system represents an evolution of a 135 previous version developed by the Remote Sensing Laboratory 


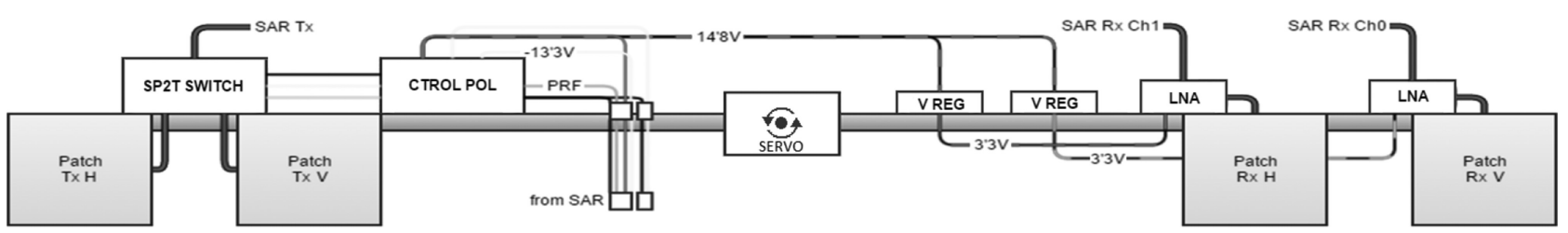

Fig. 4. Schematic of the transmitter/receiver RF front end.

TABLE I

ARBRES-X SAR SENSOR PARAMETERS

\begin{tabular}{lc}
\hline \hline System Parameters & X-Band \\
\hline Carrier Frequency $\left(f_{0}\right)$ & $9.65 \mathrm{GHz}$ \\
Chirp Sampling Frequency $\left(f_{s}\right)$ & $44.62 \mathrm{MHz}$ \\
Chirp P RF & $5.44 \mathrm{KHz}$ \\
Chirp Bandwidth & $100 \mathrm{MHz}$ \\
Transmitted Power & $30 \mathrm{dBm}$ \\
Range Resolution (nominal) & $1.5 \mathrm{~m}$ \\
Weight & $\approx 2 \mathrm{~kg}$ \\
Power Consumption & $29.3 \mathrm{~W}$ \\
\hline
\end{tabular}

of the UPC [4], [5]. It is a SAR sensor operating at $9.65 \mathrm{GHz}$ that works with a stepped-linear-frequency-modulated continuouswave signal. The complete system has been designed to be fitted in small UAVs, as is the case of the UAV MP. This has imposed strong constraints in its design in terms of weight, power consumption, compactness, and robustness. The main characteristics of the ARBRES-X SAR sensor are summarized in Table I, and the block diagram of the system is shown in Fig. 3.

The sensor can be divided into four units: the frequency generation unit (FGU), the transmitter, the receiver, and the baseband unit. The fully polarimetric capability is achieved with an array of $2 \times 2$ patch antennas transmitting and receiving in both vertical and horizontal channels. The microstrip antennas present a beamwidth of approximately $36^{\circ}$ in elevation and $42^{\circ}$ in azimuth. A solid-state switch is used to switch between the two transmitting antennas, changing the polarization for every transmitted chirp signal. Fig. 4 shows the RF front end, consisting of a lightweight aluminum bar together with the patch antennas, which has been specifically designed to be integrated into the UAV MP. In the image, the abbreviations SP2T and VREG correspond to single-pole double-throw and voltage regulator, respectively.

The receiver unit takes advantage of the use of the dechirpon-receive technique to reduce its complexity [6]. It consists of two parallel low-noise chains with a direct zero-IF demodulator, where a sample of the transmitted signal is used as a local oscillator. To reduce the noise and to amplify the signal in the receiver, a low-noise amplifier is directly connected to the antenna. After the baseband conversion, the signal acquisition in the baseband unit is performed by a commercial high-speed digitizer controlled by a single-board computer with a solid-state hard disk drive. The two baseband signals are synchronously digitized using trigger and clock references that are coherently generated in the FGU.

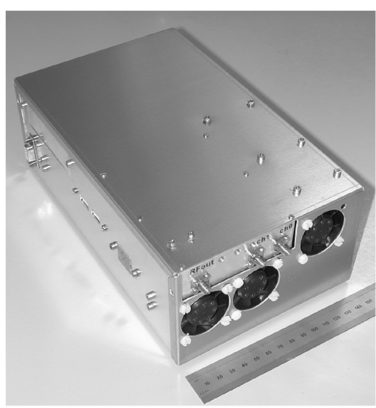

(a)

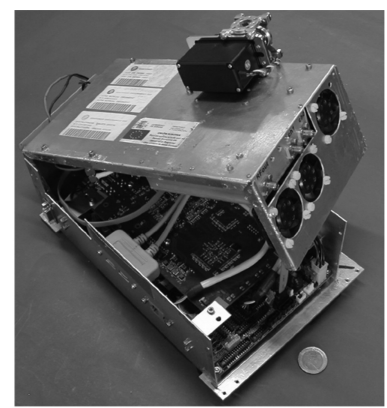

(b)

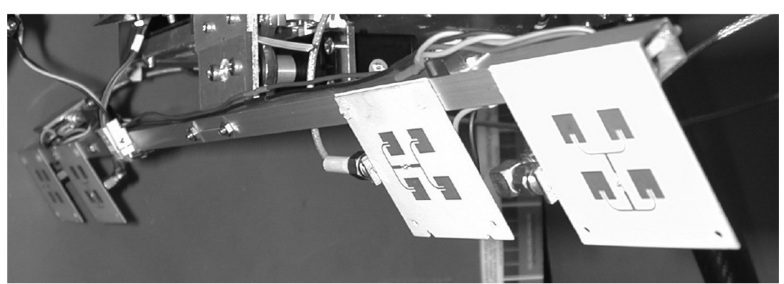

(c)

Fig. 5. (a) and (b) ARBRES-X SAR sensor. (c) Lightweight aluminum bar together with the patch antennas.

Fig. 5 shows the compacted lightweight ARBRES-X SAR and the transmitter and receiver antennas integrated in the system. A servo motor, together with a simple IMU, holds the lightweight aluminum bar. Its purpose is to maintain the antennas in horizontal position with respect to the ground, compensating the tilt angle of the platform while flying.

The area illuminated by the ARBRES-X SAR sensor is extended to approximately $1500 \mathrm{~m}$ in range and $900 \mathrm{~m}$ in azimuth. The SLC images obtained from the retrieved data are processed offline with the backprojection algorithm (BPA) [7]. In this context, the received baseband signal is subsequently range compressed applying the fast Fourier transform, and then, the BPA algorithm is applied in order to perform the azimuth focusing. In spite of its high computational cost, the BPA offers a high degree of flexibility in focusing extended images from arbitrary synthetic apertures, limited either by antenna beam, the linear track, or by the data acquisition capacity [4]. Finally, the range resolution obtained with the parameters used by the ARBRES-X SAR sensor is approximately $1.5 \mathrm{~m}$, and the cross-range resolution depends on the type of measurement.

\section{UAV MP SAR EXPERIMENTAL RESULTS}

In this section, preliminary results obtained with the 193 ARBRES-X SAR sensor integrated in the UAV MP are 194 


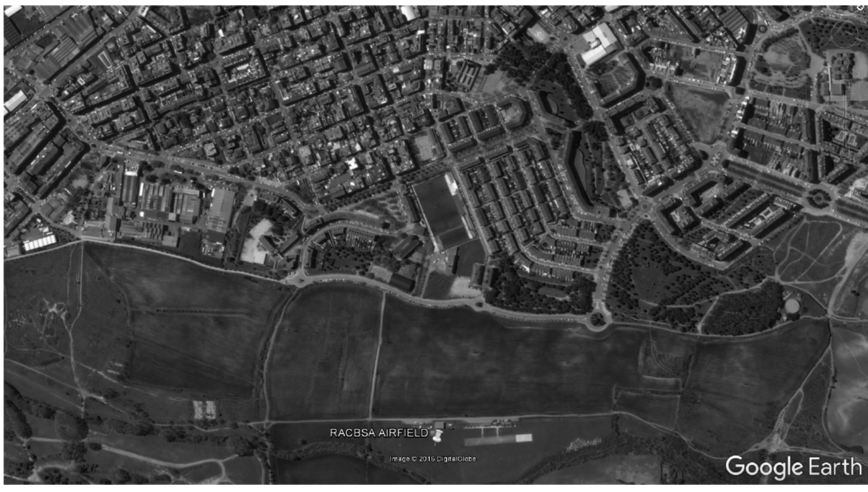

Fig. 6. Aerial photograph of the scenario from Google Earth.

presented. The aim is to explore the SAR capabilities of this type of platforms.

\section{A. Test Site Description}

The scenario selected to test the system and to perform several measurements is the Real Aero Club BarcelonaSabadell (RACBSA) Radio Control Airfield (Montcada i Reixac, Barcelona, Spain). The scenario contains agricultural fields surrounded by an urban area with smooth topography, as shown in Fig. 6. It is mainly composed of elements with high reflectivity, such as buildings, roads, pylons, or parked cars, which make it suitable to validate the system performances.

\section{B. Stability of the UAV MP SAR Platform and Application of the Autofocus Algorithm}

In Section II, the inherent instability of the platform has been mentioned, which can degrade the quality of the retrieved data from the ARBRES-X SAR sensor. The deviations respect to the nominal flight track impose severe restrictions on the maximum processable aperture length. The larger the aperture, the greater the cumulative positioning error of the platform with respect to the ideal flight path, and the greater the degree of defocusing in the retrieved images. Fig. 7 shows a comparison of three SLC images of the scenario obtained with different aperture lengths. Cartesian coordinates are used because it is visually easier to compare the SLC images with the real scenario. As it is evidenced, the defocusing increases for larger apertures, where the flight path is more susceptible to have deviations from the nominal trajectory. Considering the coordinate system presented in Fig. 2(a), an error in the positioning in the $x$-axis is due to errors in the velocity of the platform, which is equivalent to a nonuniform azimuth sampling of the signal. Errors in the $z$-axis are related to the height of the platform and in the $y$-axis with the lateral deviation of the platform with respect to the scenario. The defocusing of the image in Fig. 7(c) can be appreciated in the loss of resolution of the three PSs corresponding to polarimetric active radar calibrators (PARCs) located in the near range center of the image, together with the blurring effect in far range.

The aperture length is related to the azimuth resolution of the SAR system and to the signal-to-clutter ratio (SCR) of the focused images, since the energy emitted is greater for larger 233 apertures. Thus, in order to process larger apertures, it is necessary to compensate for the undesired effects of the nonidealities in the platform flight path. In this context, the first attempt was to incorporate an IMU integrated with a global positioning satellite (GPS) receiver that has a position accuracy of $2.5 \mathrm{~m}$ and an angular dynamic accuracy of $1^{\circ}$ RMS. The position and attitude information of the platform were logged simultaneously with the radar raw data for a proper image geocoding. The information provided by the IMU was intended to be used to apply the motion compensation (MoCo) technique in the focusing process. Thus, the possible motion errors could be compensated, and the defocusing, the geometric distortions, and the phase errors of the retrieved images could be minimized. Nevertheless, this option was discarded because the accuracy of cost-effective devices that are suitable to be integrated in the UAV MP, overcoming restrictions of size and weight, is not enough to fulfill the requirements needed. In this sense, the accuracy of these systems is too low in terms of the wavelength. Besides, the vibrations of the platform are another important drawback that degrades the quality of the measurements performed by the IMU.

To overcome the defocusing problem due to the instabilities of the UAV MP when processing long apertures, an autofocus algorithm has been applied. Different techniques have been proposed in the literature that are used to compensate for motion errors in SAR imagery [8], [9]. In our case, an algorithm based on the retrieved phase history of three PSs is applied with the objective to derive the deviations of the platform in the three axes [10]. Since it is difficult to find three natural PSs in the scenario with good reflectivity response, three different PARCs have been placed in visible and accessible locations of the scenario in order to validate the autofocus algorithm. Fig. 8 shows one of the PARCs placed in the agricultural field of the scenario, surrounded of growing vegetation. Their pointing angles (azimuth and elevation) are adjusted to maximize their visibility with the sensor. They can be recognized in Fig. 7 as the three PSs forming a triangle between 150 and $220 \mathrm{~m}$ in the range axis. The phase evolution of one PARC along the aperture can be expressed as

$$
\varphi_{\mathrm{PARC}}(t)=\frac{4 \pi R_{\mathrm{PARC}}(t)}{\lambda}
$$

where $\lambda$ is the wavelength and $R_{\text {PARC }}(t)$ is the slant range dis- 272 tance from the sensor to the PARC during the slow time $t$, i.e., the 273 distance from the sensor to the PARC for every antenna position 274 during the acquisition time. Analyzing the evolution of the phase 275 of the PARC $\varphi_{\mathrm{PARC}}(t)$ during the acquisition time, the relative 276 distance variation from the sensor to the PARC $R_{\text {PARC }}(t)$ can 277 be obtained from (1), but not the nominal value of the distance. 278 In practice, it can be extrapolated for the entire aperture know- 279 ing the slant range distance at the first instant of measurement 280 $R_{\text {PARC }}(t=0)$. Despite not having the real value $R_{\text {PARC }}(t=0), \quad 281$ it can be approximated by its ideal value taking as reference the 282 position of the platform given by the GPS. This approximation 283 is valid because the positioning errors of the platform are small 284 in comparison with the distance from the sensor to the scatterer. 285 


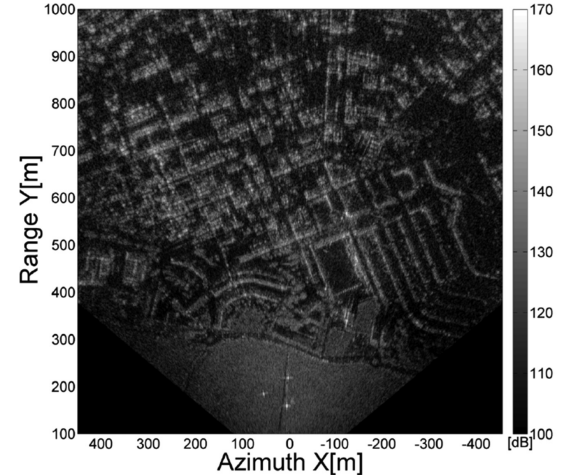

(a)

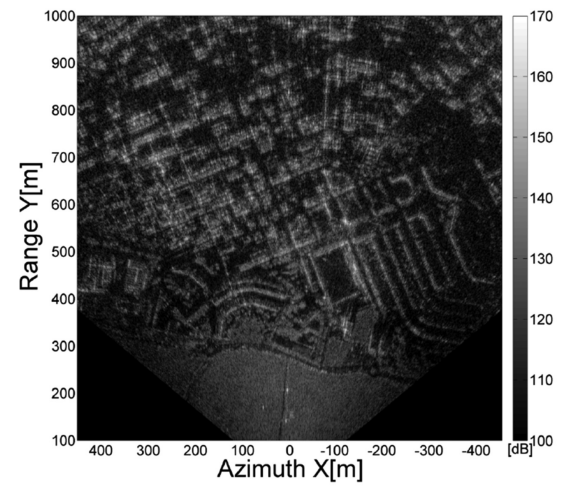

(d)

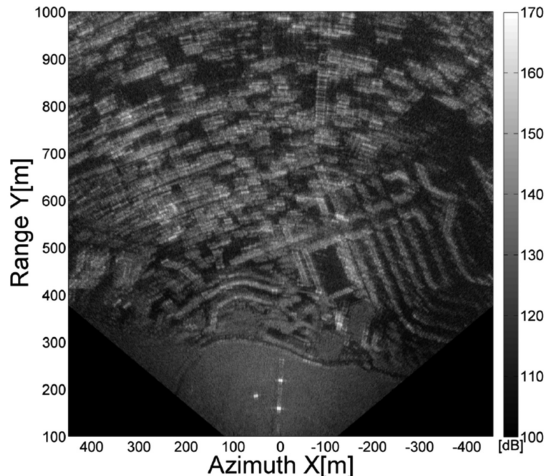

(b)

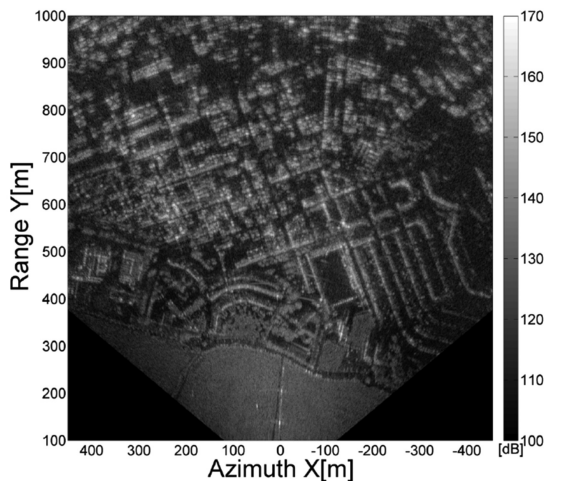

(e)

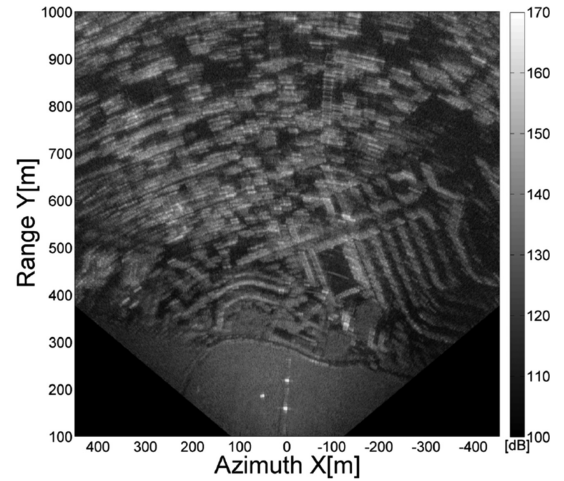

(c)

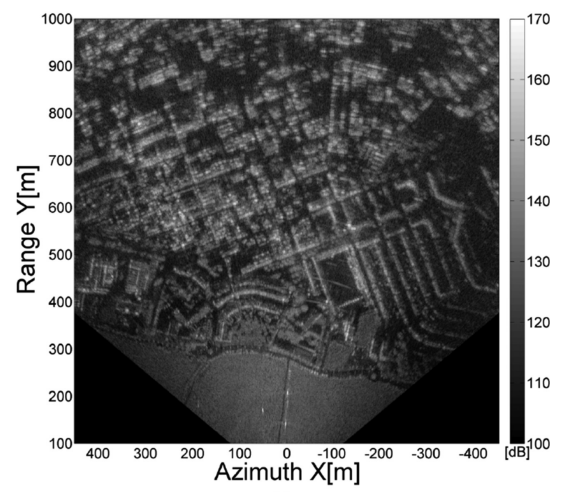

(f)

Fig. 7. SLC images in Cartesian coordinates for different aperture lengths before autofocus: (a) $13.4 \mathrm{~m}$, (b) $40.9 \mathrm{~m}$, and (c) $55.6 \mathrm{~m}$. Different aperture lengths after autofocus: (d) $13.4 \mathrm{~m}$, (e) $40.9 \mathrm{~m}$, and (f) $55.6 \mathrm{~m}$. Flight conditions: $150 \mathrm{~m}$ height, $v_{\mathrm{UAV}}=8.9 \mathrm{~m} / \mathrm{s}, \mathrm{VV}$ Pol.

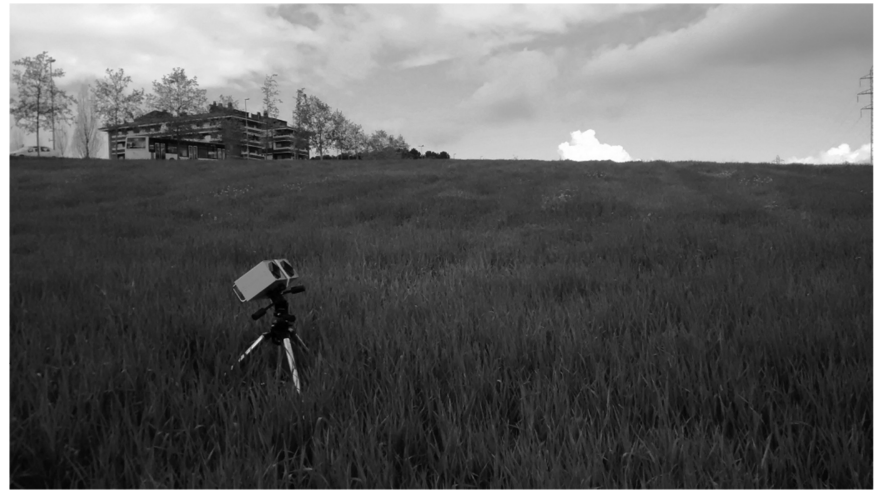

Fig. 8. PARC location in the agricultural field of the scenario.
The relative distance $R_{\text {PARC }}(t)$ can be expressed by the quadratic function as

$$
\begin{aligned}
R_{\mathrm{PARC}}(t)= & \left(\left(x_{\mathrm{PARC}}-x_{a}(t)-u_{x}(t)\right)^{2}+\right. \\
& \left.+\left(y_{\mathrm{PARC}}-u_{y}(t)\right)^{2}+\left(z_{\mathrm{PARC}}-u_{z}(t)\right)^{2}\right)^{1 / 2}
\end{aligned}
$$

where $x_{\text {PARC }}, y_{\text {PARC }}$, and $z_{\text {PARC }}$ correspond to the ideal position of the PARC in the $x-, y$-, and $z$-axis respectively, $x_{a}(t)$ is the azimuth antenna position during the slow time, and $u_{x}(t)$, $u_{y}(t)$, and $u_{z}(t)$ are the positioning errors of the platform in 291 the three axes with respect to the nominal flight path. Having 292 three PARCS, a system of three nonlinear equations and the three unknowns can be solved for every azimuth position of the platform. Thus, the deviations of the platform $u_{x}(t), u_{y}(t)$, and $u_{z}(t)$ can be derived to refocus the image taking these variations into account. The values obtained for the deviations of the platform must be filtered to avoid undesired effects of blurring in the compensated image. This is necessary because the retrieved parameters can present some noise that could be introduced by irregularities in the phase or by inaccuracies when solving the equations. Because of that, they must be filtered to prevent SLC images from being degraded when introducing the coefficients in the BPA. In our case, to perform the filtering, a moving average low-pass filter is used. Fig. 9 shows the retrieved information from the PARCs phase and the computed parameters to perform the autofocus.

Fig. 7(d)-(f) shows the three different SLC images obtained in Fig. 7(a)-(c) after applying the autofocus algorithm. The correction parameters $u_{x}(t), u_{y}(t)$, and $u_{z}(t)$ obtained in Fig. 9 are introduced in the focusing process to generate the images. Comparing Fig. 7(c) and (f), it is clearly visible that after applying the autofocus algorithm, the images do not show the azimuth defocusing for large apertures. Moreover, the fact of processing larger apertures implies a higher transmitted energy, i.e., higher SCR for deterministic targets, and an improvement in the quality 


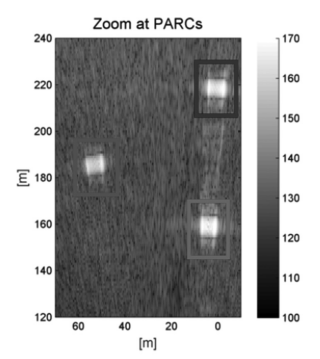

(a)

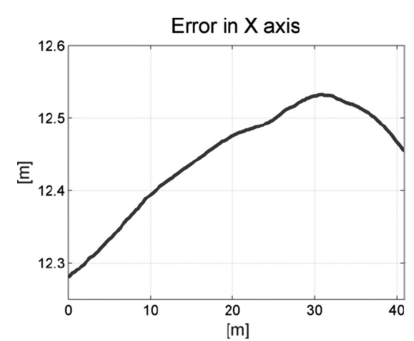

(e)

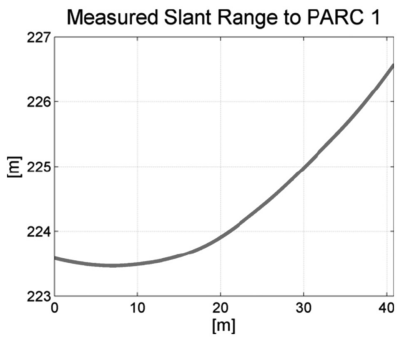

(b)

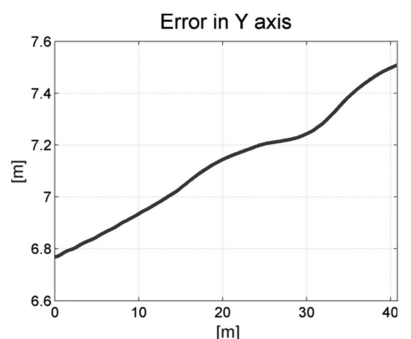

(f)

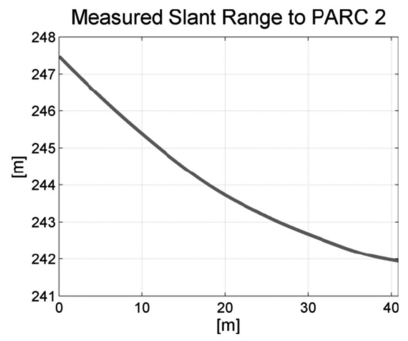

(c)

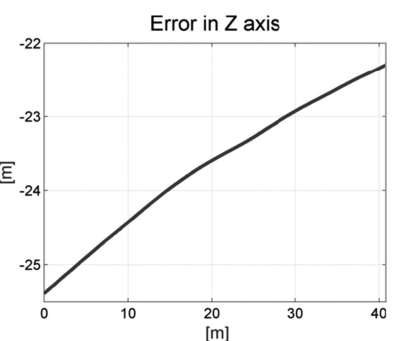

(g)

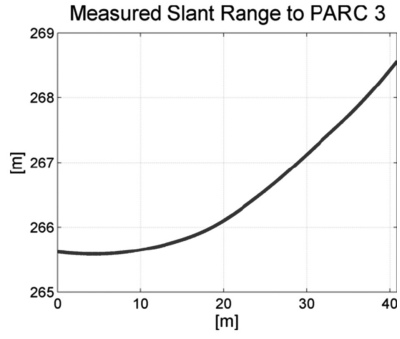

(d)

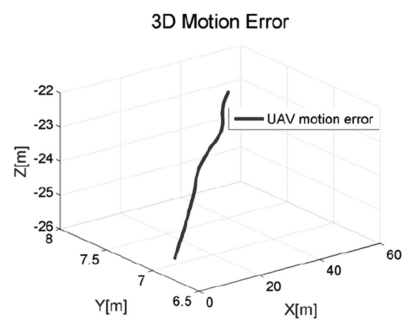

(h)

Fig. 9. Autofocus parameters for aperture of approximately $40.9 \mathrm{~m}$ and flight conditions: $150 \mathrm{~m}$ height, $v_{\mathrm{UAV}}=8.9 \mathrm{~m} / \mathrm{s}$, VV Pol. (a) SLC image in Cartesian coordinates with zoom to PARCs. Relative measured slant range to (b) PARC 1, (c) PARC 2, and (d) PARC 3 . Filtered deviation: (e) $u_{x}(t)$, (f) $u_{y}(t)$, and (g) $u_{z}(t)$. (h) Three-dimensional representation of the platform deviations.

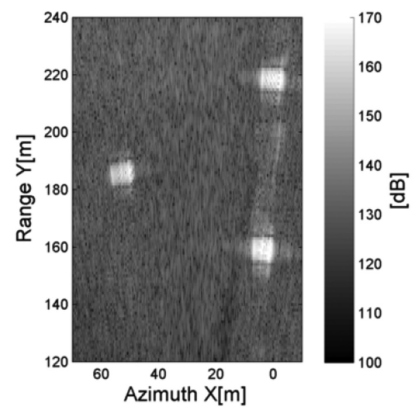

(a)

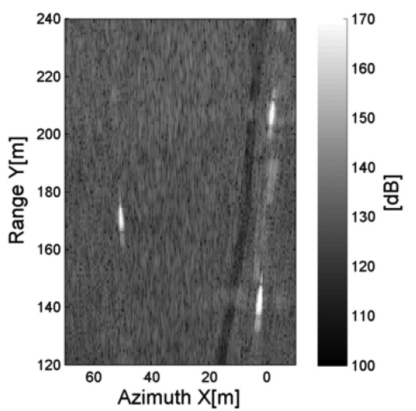

(b)

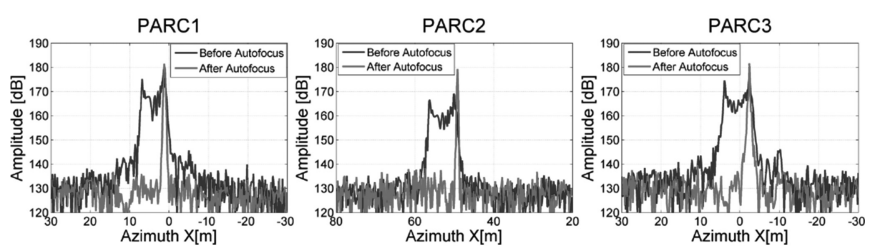

(c)

Fig. 10. Aperture length of $40.9 \mathrm{~m}$. Zoom to PARCs in SLC image (a) before and (b) after application of the autofocus algorithm. (c) Cut in azimuth of the PARCs before and after application of the autofocus algorithm. of the SLC images. The improvement in the azimuth resolution after applying the autofocus algorithm can be clearly seen in Fig. 10, where a zoom to the area containing the PARCs in the SLC images is shown. Even the track in the scenario is well defined in the image of Fig. 10(b), while in Fig. 10(a), it is not easily recognizable. The cut in azimuth of the three PARCs can be seen in Fig. 10(c). The response of the three PSs is clearly spread before applying the autofocus algorithm and gets better focused after applying it. Thus, the redistribution of the energy
TABLE II

AZIMUTH RESOLUTION

\begin{tabular}{lcc}
\hline \hline & $\Delta_{\mathrm{az}}$ Theoretical $[\mathrm{m}]$ & $\Delta_{\mathrm{az}}$ Measured [m] \\
\hline PARC 1 & 0.14 & 0.39 \\
PARC 2 & 0.16 & 0.22 \\
PARC 3 & 0.16 & 0.20 \\
\hline \hline
\end{tabular}

in the focusing process results in higher values of amplitude 326 for the peak associated with the PARC, in particular 2.31, 10.2, 327 and $6.08 \mathrm{~dB}$, for PARCs 1,2, and 3, respectively. The use of an 328 aperture length shorter than the dimensions of the illuminated 329 scenario implies that the azimuth resolution $\Delta_{a z}$ will depend 330 on the range distance $R$, the length of the synthetic aperture $L, \quad 331$ and the squint angle $\theta$ as

$$
\Delta_{\mathrm{az}}=\frac{\lambda R}{2 L \cos ^{2}(\theta)} .
$$

Table II summarizes the theoretical and measured resolutions 333 after the autofocus algorithm for the aperture of $40.9 \mathrm{~m}$. By 334 means of (3), the theoretical azimuth resolutions of PARCs 335 1,2 , and 3 can be calculated, being $0.14,0.16$, and $0.16 \mathrm{~m}, 336$ respectively. Moreover, the measured 3-dB azimuth resolutions 337 after applying the autofocus algorithm are 0.39, 0.22, and 0.20338 $\mathrm{m}$ for PARCs 1,2 , and 3, respectively. It has to be considered 339 that, in order to reduce the secondary lobes that may degrade the 340 quality of the focused image, a Hanning filter window has been 341 applied in the cross-range domain, degrading the main lobe. 342 Taking this issue into account, the measured azimuth resolutions 343 after applying the autofocus algorithm are quite close to the 344 theoretical ones, although the autofocus algorithm could still be 345 improved. 


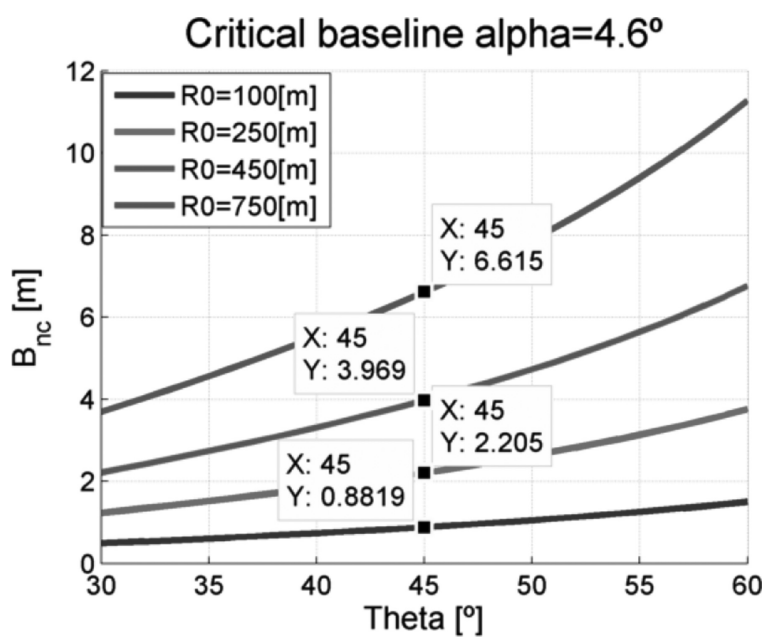

Fig. 11. Critical baseline as a function of the off-nadir angle for different $r_{0}$.

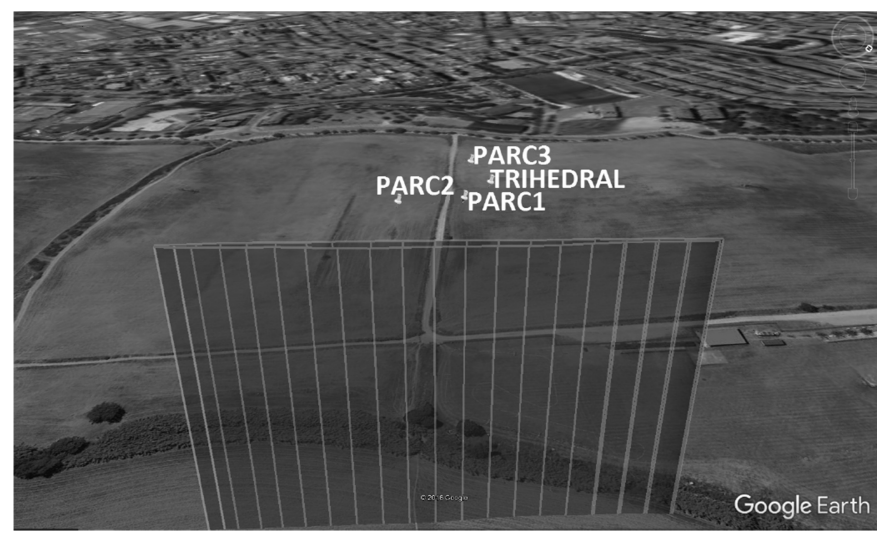

Fig. 12. Image of the scenario taken from Google Earth with the flight path information and the different calibrators.

The capability of the ARBRES-X SAR sensor integrated in the UAV MP to obtain SLC images has been demonstrated in this section.

\section{Innovative Flight Strategies in Airborne UAV MP Multidimensional SAR}

As has been pointed out previously, the flight capabilities of the UAV MP open new possibilities in airborne SAR observations. The present section exposes the repeat-pass interferometric and the fully polarimetric results obtained with the ARBRES-X SAR sensor integrated in the UAV MP platform. Furthermore, some results of measurements obtained performing vertical and circular flights are presented. The aim of this section is to assess the system performance and the SAR capabilities of the UAV MPs.

1) UAV MP SAR Repeat-Pass Interferometry: SAR interferometry exploits the phase differences between two sets of complex SAR data to derive, for example, topographic information. Differential SAR interferometry is a particular configuration, in which the interferometric data are obtained in different passes separated by a short baseline for deformation measurements

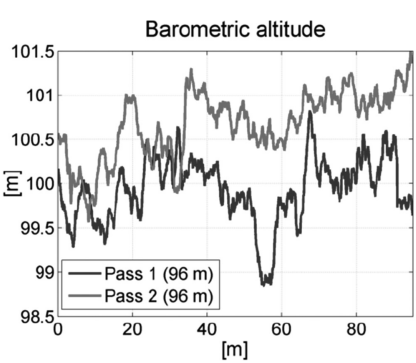

(a)

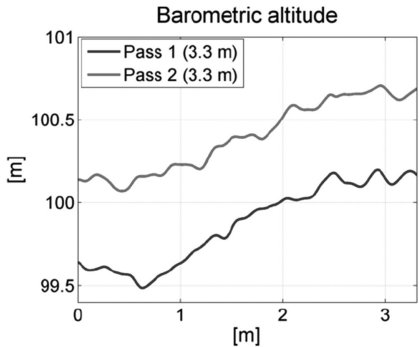

(c)

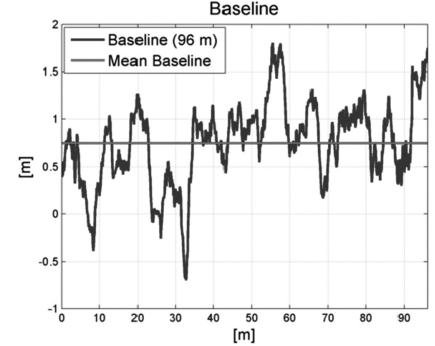

(b)

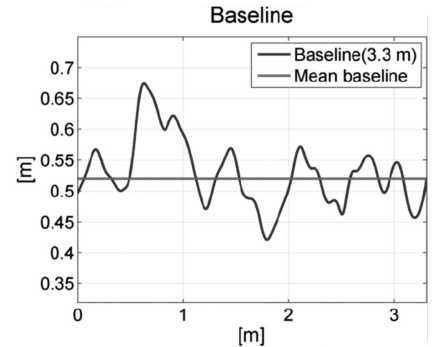

(d)
Fig. 13. Barometric altitude (a) and baseline (b) of the two passes of an aperture of approximately $96 \mathrm{~m}$ and (c) and (d) of an aperture of approximately $3.3 \mathrm{~m}$.

[11]. The antenna position that defines the baseline during the two passes is of great importance to have a good accuracy in the interferometric process. In the UAV MP, the baseline depends on the precision in positioning to perform the required flight paths and the undesired motion errors. Having a good control of the trajectory, together with the minimization of the instabilities of the platform, is mandatory to obtain interferometric results. According to [12], the total baseline decorrelation is reached in correspondence with the critical baseline

$$
\left|B_{n c}\right|=\left|\frac{\lambda r_{0} \tan (\theta-\alpha)}{2 \rho_{s}}\right|
$$

where $\lambda$ is the wavelength of the system, $r_{0}$ is the sensor to 376 target distance, $\theta$ is the off-nadir angle, $\alpha$ is the local terrain 377 slope (range), and $\rho_{s}$ is the slant range resolution. Fig. 11378 shows the evolution of the critical baseline as a function of $\theta$ for 379 different $r_{0}$.

In our particular case, the off-nadir angle of the ARBRES$\mathrm{X}$ SAR system integrated in the UAV MP is approximately $45^{\circ}$ and the local terrain slope of the illuminated scenario is approximately $4.6^{\circ}$ with an inclination of the $8 \%$ up to $300 \mathrm{~m}$. In Fig. 11, it can be seen that, for $r_{0}=100 \mathrm{~m}$ and $r_{0}=750 \mathrm{~m}$, the critical baseline is 0.88 and $6.6 \mathrm{~m}$, respectively.

Taking advantage of the possibility offered by the UAV MP to perform preprogrammed routes, repeat-pass interferometry strategies can be assessed. Fig. 12 shows an aerial view of the scenario with the flight path information, where two different passes of approximately $96 \mathrm{~m}$ in the stripmap configuration can be seen. The UAV MP was programmed to fly at $100 \mathrm{~m}$ with an interferometric baseline between passes of $0.8 \mathrm{~m}$, which is under the critical baseline calculated by (4) for $r_{0}=100 \mathrm{~m}$.
78 


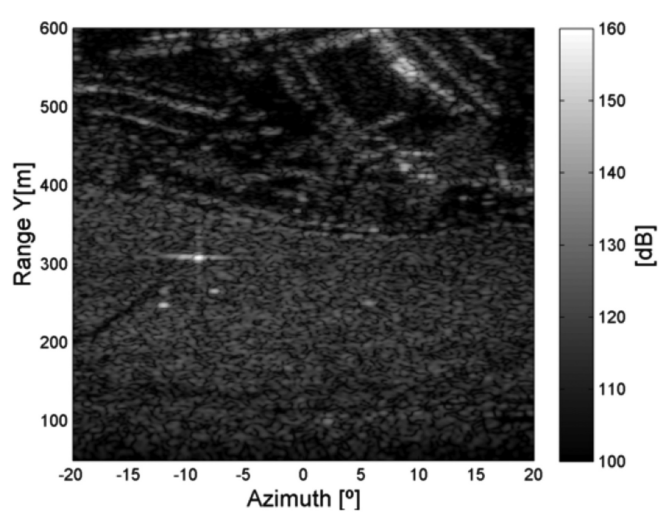

(a)

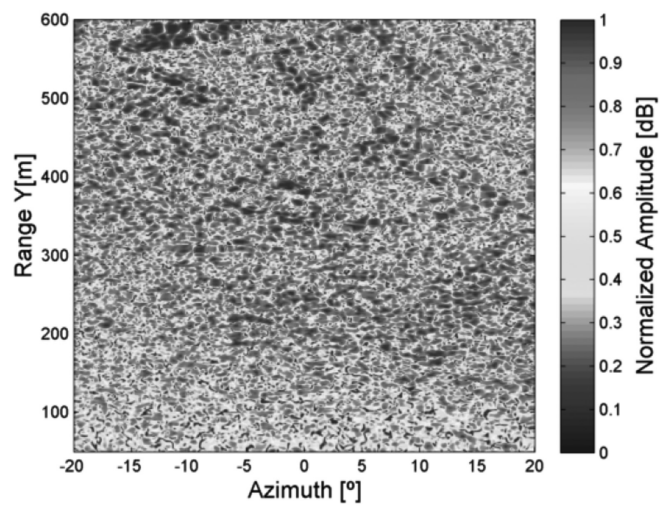

(c)

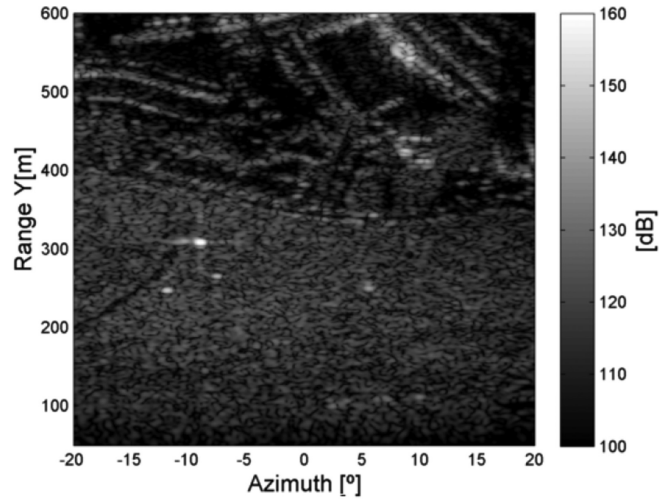

(b)

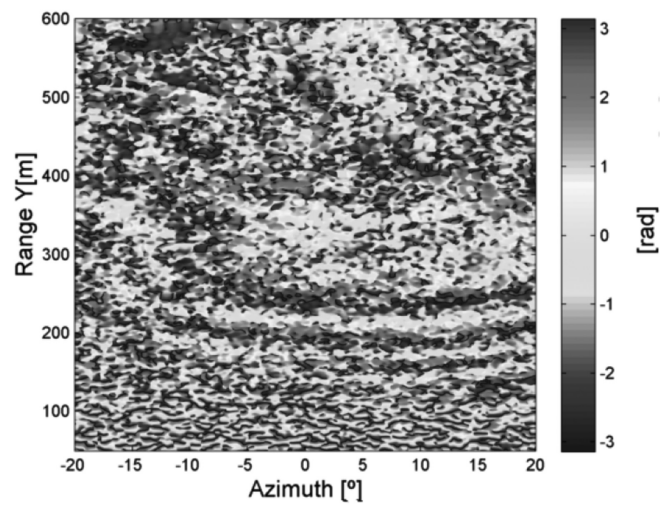

(d)

Fig. 14. Polar coordinates. (a) SLC pass 1. (b) SLC pass 2. (c) Coherence multilook $4 \mathrm{~m} \times 0.4^{\circ}$. (d) Interferogram multilook $4 \mathrm{~m} \times 0.4^{\circ}$. Flight conditions: 100 -m height, $v_{\mathrm{UAV}}=8.7 \mathrm{~m} / \mathrm{s}, 0.52-\mathrm{m}$ mean baseline, VV Pol.

Three PARCs and a trihedral were placed in the agricultural field to be used as references.

Fig. 13(a) and (b) shows the evolution of the barometric altitude and the interferometric baseline of the UAV MP, respectively, for the complete aperture of $96 \mathrm{~m}$. The mean baseline of the aperture is $0.74 \mathrm{~m}$, which is under the critical baseline. As it is clearly visible, the height of the platform was not constant during the acquisition time, which introduces a lot of focusing errors in the processing. Because of that, shorter apertures have to be processed to minimize the impact of the flight deviations and instabilities in the retrieved images, such as phase errors. The autofocus algorithm cannot be applied yet to compensate this motion errors in long apertures. This is because the correction is not as precise as needed in terms of the wavelength. Fig. 13(c) shows the barometric altitude for an aperture of approximately $3.3 \mathrm{~m}$, where the measurements are less affected by the instabilities of the UAV MP during the flight path. Furthermore, the selected part of the aperture for the two passes has a similar ascending behavior. Finally, Fig. 13(d) shows the interferometric baseline evolution for this shorter aperture, where the largest difference between the maximum and the minimum is $0.25 \mathrm{~m}$ and the mean baseline is $0.52 \mathrm{~m}$. At this point, it is worth mentioning that the theoretical accuracy of the navigation system in the positioning of the platform is $\pm 0.5 \mathrm{~m}$ in vertical and $\pm 1.5 \mathrm{~m}$ in horizontal. Furthermore, the velocity accuracy of the
UAV MP is $0.1 \mathrm{~m} / \mathrm{s}$, which is determined by the GPS. Moreover, 420 the system incorporates a barometer that has a vertical accuracy 421 of nearly $25 \mathrm{~cm}$. Despite these values, the parameters retrieved 422 from the IMU of the platform do not have enough accuracy to 423 quantify the precision of the flights. This can be observed, for 424 instance, in the data shown in Fig. 13, where the response of the 425 IMU represents unreal dynamics of the platform. An example 426 can be seen in the barometric altitude shown in Fig. 13(a), where 427 we can notice impossible variations in the height of the platform 428 of $1.25 \mathrm{~m}$ in $0.15 \mathrm{~s}$.

Fig. 14(a) and (b) shows the SLC images obtained with the 430 two different passes. The coherence and the retrieved interfer- 431 ometric phase are shown in Fig. 14(c) and (d), respectively. In 432 this case, the velocity during the short aperture has been as- 433 sumed constant, which is a feasible approximation, since the 434 acquisition time of $0.3 \mathrm{~s}$ is too short to allow great velocity 435 deviations.

As can be seen in Fig. 13, the motion errors of the platform in 437 each pass are independent during the interferogram generation. 438 In this case, differently from single-pass systems, the baseline 439 has a time-varying error that may cause significant phase errors 440 in range and azimuth direction. Nevertheless, Fig. 14 shows that, 441 despite having a nonconstant baseline during the selected part 442 of the aperture, the first interferogram with a UAV MP has been 443 obtained. However, it has to be admitted that it is extremely 444 


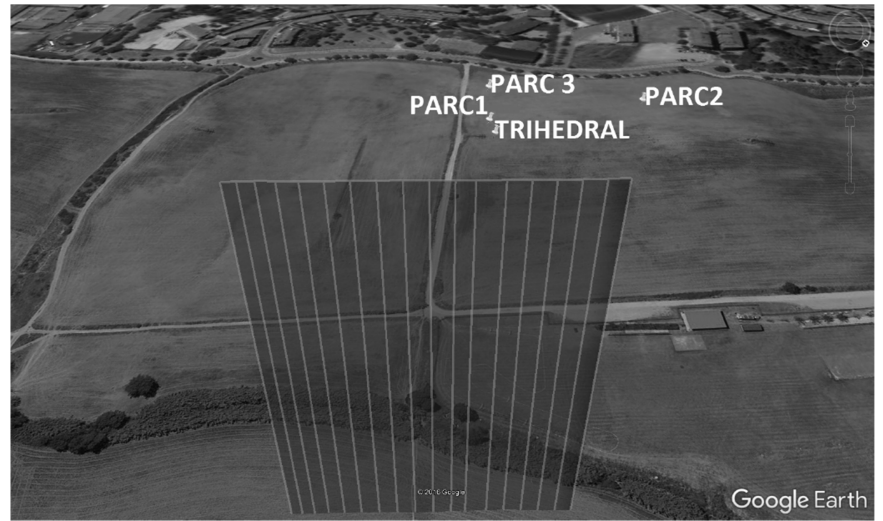

Fig. 15. Disposition of the calibrators in the scenario and flight path performed by the UAV MP. Image from Google Earth.

difficult to obtain interferometric maps, since perfectly parallel and aligned passes are difficult to achieve with the UAV MP. This is due to the lack of the required accuracy in the positioning of the platform and its inherent motion errors.

As the accuracy of the GPS and the IMU is not sufficient to retrieve the flight deviations that permit to let us know the correct baseline during the acquisition time, future steps include devoting some effort on the estimation and correction of timevarying baseline errors from the interferometric SAR data to improve the results, applying methods such as the one proposed in [13]. Moreover, a better navigation system operating with RTK GPS together with an IMU should be used, which would permit an accuracy of few centimeters both in vertical and horizontal. This way, larger apertures could be processed, and better interferometric results could be achieved.

2) Fully Polarimetric UAV MP SAR Measurements: To evaluate the fully polarimetric capabilities of the ARBRES-X SAR sensor, some measurements have been performed in the RACBSA airfield. To analyze the results, it has to be taken into account that, as said in Section II, four patch antennas have been used in the system. They have been selected for their simplicity, low weight, low size, and easy integration into the system, but they usually offer low levels of crosstalk. Despite this difficulty, the cross-polarization discrimination factor of the patch antennas is approximately $-22 \mathrm{~dB}$ [4]. Furthermore, the isolation between the two receiving channels is approximately $-30 \mathrm{~dB}$. Considering this, the first preliminary results presented in this section are not polarimetrically calibrated, but, as will be shown, the theoretical and measured results are consistent. In any case, the required calibration will not alter excessively the results.

Fig. 15 shows the flight path performed by the UAV MP and the disposition of different calibrators in the scenario. To assess the different polarizations, three PARCs and a trihedral corner reflector have been placed in the agricultural field of the area. Fig. 16(a) and (b) shows the photograph of PARC 1 and PARC 2, respectively. Fig. 16(c) shows PARC 3, which has been rotated $45^{\circ}$. Finally, the trihedral corner reflector is shown in Fig. 16(d).

The theoretical scattering matrices of the different elements are shown in (5), and the theoretical polarization signatures are shown in Fig. 17. The trihedral corner reflector is expected to

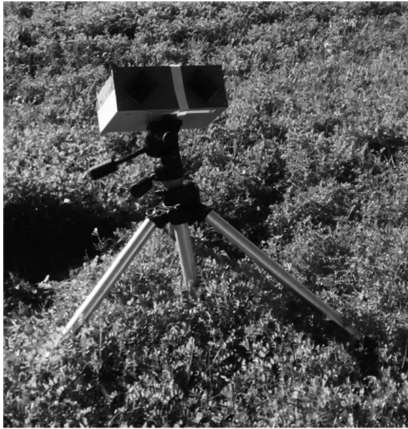

(a)

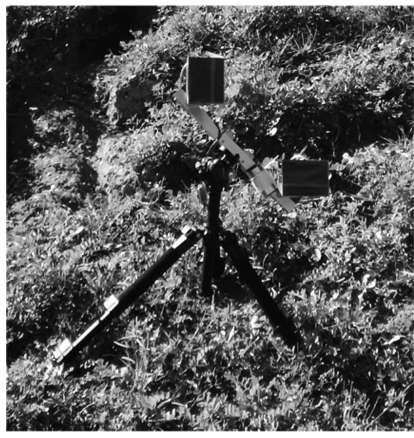

(c)

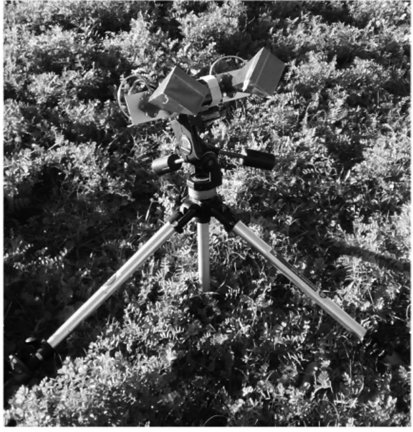

(b)

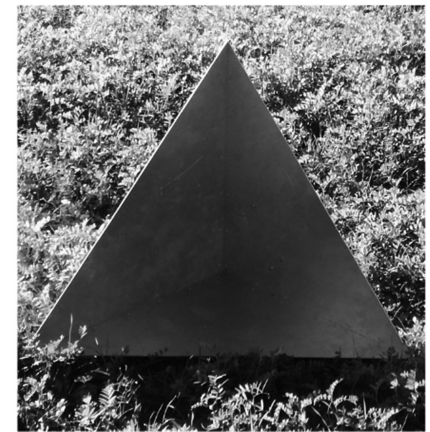

(d)
Fig. 16. Calibrators placed in the scenario. (a) PARC 1. (b) PARC 2 (c) PARC 3. (d) Trihedral corner reflector.

have identical horizontal and vertical backscattering sections, $\mathrm{HH}=\mathrm{VV}$, while no cross-polarization components are generated, $\mathrm{HV}=\mathrm{VH}=0$. PARC 1 and PARC 2 are expected to have cross-polarization and copolarization components, and, finally, PARC 3 is expected to have only the cross-polarization component $\mathrm{HV}$

$$
\begin{aligned}
S & =\left(\begin{array}{c}
S_{\mathrm{HH}} S_{\mathrm{HV}} \\
S_{\mathrm{VH}} S_{\mathrm{VV}}
\end{array}\right) \\
S_{P 1-2} & =\left(\begin{array}{ll}
1 & 1 \\
1 & 1
\end{array}\right), \quad S_{P 3}=\left(\begin{array}{ll}
0 & 1 \\
0 & 0
\end{array}\right), S_{T R I}=\left(\begin{array}{ll}
1 & 0 \\
0 & 1
\end{array}\right) .
\end{aligned}
$$

Fig. 18 shows the SLC images of the four polarizations $\mathrm{HH}$, $\mathrm{HV}, \mathrm{VH}$, and VV. As expected, PARC 1 and PARC 2 can be perfectly seen in all the polarizations, while the cross-polar polarization $\mathrm{HV}$ is the most important component of PARC 3. Besides, the most important components of the measured trihedral are $\mathrm{HH}$ and VV polarizations.

To evaluate the response of the calibrators to the different polarizations, the measured radar cross section (RCS) is shown in Fig. 19, and the retrieved scattering matrix can be seen in (6), which is derived from the measured RCS. Besides, the measured polarimetric signature of the calibrators obtained from 
Co-polarization signature of PARC1-2 Cross-polarization signature of PARC1-2
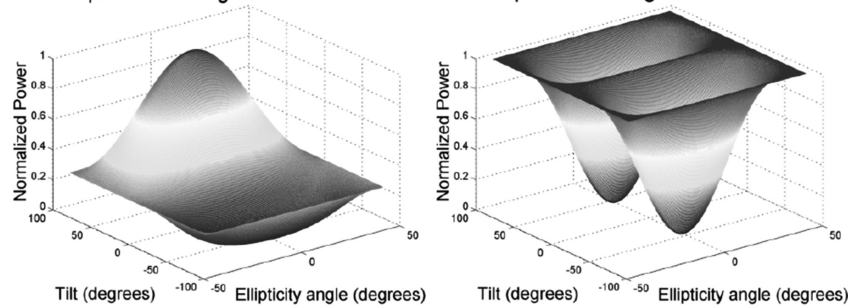

(a)

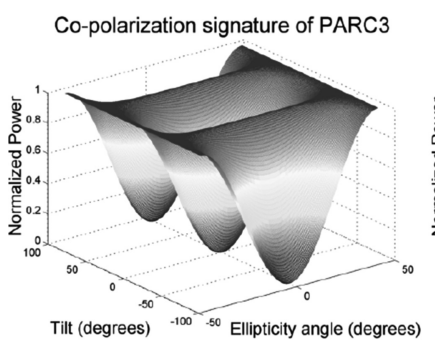

Cross-polarization signature of PARC3

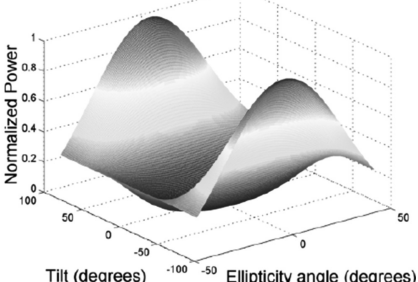

(b)

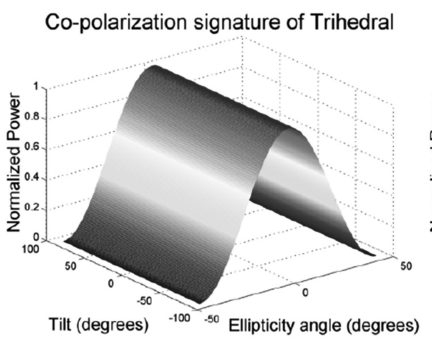

Cross-polarization signature of Trihedral

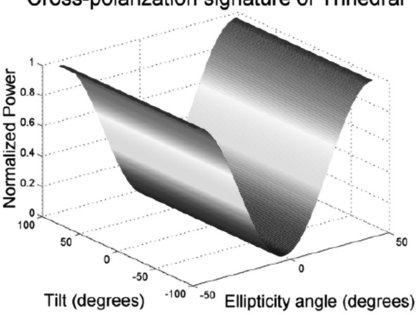

(c)

Fig. 17. Theoretical polarimetric signature of (a) PARC 1 and 2 and (b) PARC 3. (c) Trihedral corner reflector.

the scattering matrix is shown in Fig. 20

$$
\begin{array}{ll}
S_{P 1}=\left(\begin{array}{cc}
0.81 & 0.69 \\
1 & 0.78
\end{array}\right), & S_{P 2}=\left(\begin{array}{cc}
0.44 & 1 \\
0.23 & 0.49
\end{array}\right) \\
S_{P 3}=\left(\begin{array}{cc}
0.1 & 1 \\
0.02 & 0.15
\end{array}\right), & S_{\mathrm{TRI}}=\left(\begin{array}{cc}
1 & 0.08 \\
0.08 & 0.86
\end{array}\right) .
\end{array}
$$

Figs. 19 and 20 and (6) show that the polarimetric response of the different calibrators generally corresponds to the theoretical behavior, but with some variations. These differences are, in part, due to the difficulty to perfectly point the calibrators to the expected flight path. PARC 2, for example, has been placed in the scenario with an important squint angle, which makes it difficult to be well illuminated. Furthermore, the possible instabilities of the platform and the variation in the orientation of the antennas during the acquisition time can also degrade the polarimetric response.

Future steps include the polarimetric calibration of the system, which is not a trivial problem. In our system, the length of the synthetic aperture is short, and therefore, wide-beam antennas (approximately $60^{\circ}$ in our case) have to be used in order to obtain an image of suitable size. This implies a change of the polarization basis according to the illumination direction. This

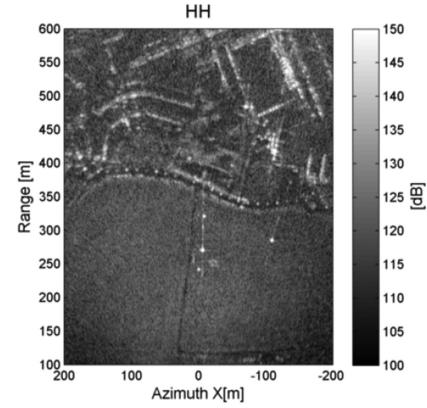

(a)

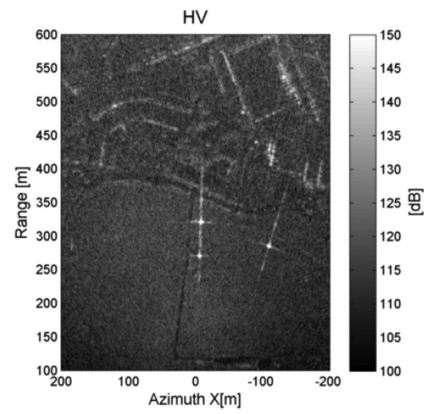

(c)

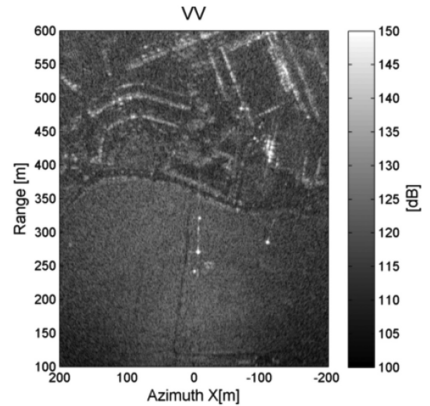

(b)

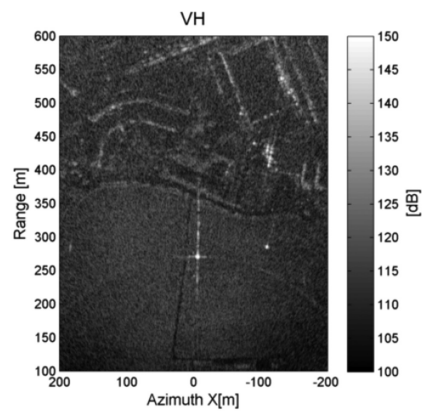

(d)
Fig. 18. SLC images of the different polarizations. (a) HH. (b) VV. (c) HV. (d) VH. Flight conditions: $150-\mathrm{m}$ height, $v_{\mathrm{UAV}}=8.9 \mathrm{~m} / \mathrm{s}$.

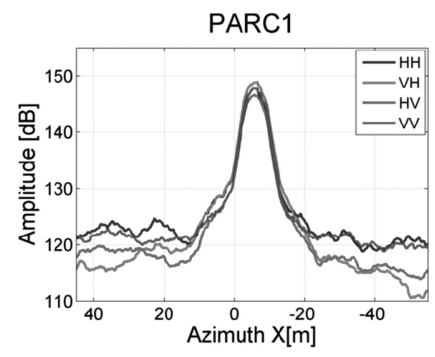

(a)

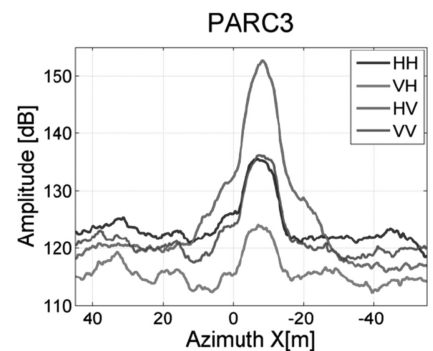

(c)

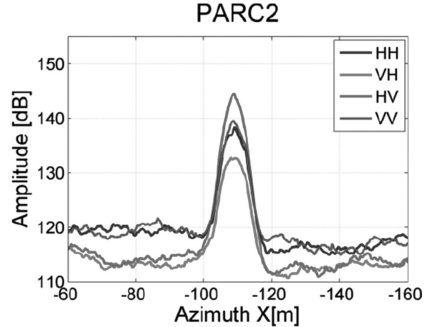

(b)

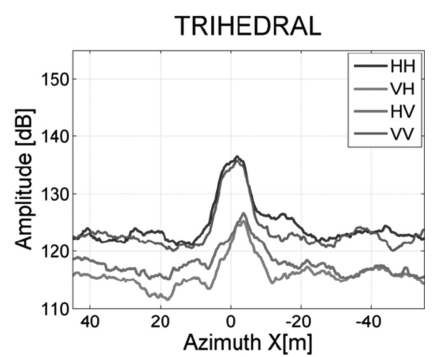

(d)
Fig. 19. Measured RCS of (a) PARC 1, (b) PARC 2, (c) PARC 3, and (d) trihedral corner reflector.

is not a problem in airborne and satellite SAR sensors, where 519 high-gain narrow beam antennas are used. As a consequence, 520 this factor must be taken into account to calibrate polarimetri- 521 cally the data.

3) Vertical Flight in UAV MP SAR: During the last years, 523 tomographic techniques for obtaining volume information of 524 


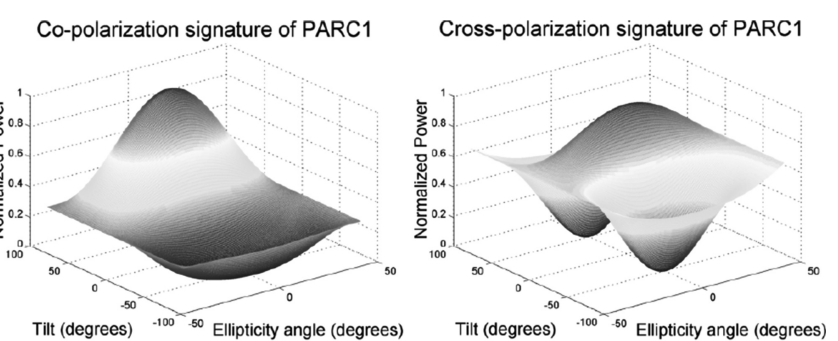

(a)

Co-polarization signature of PARC2

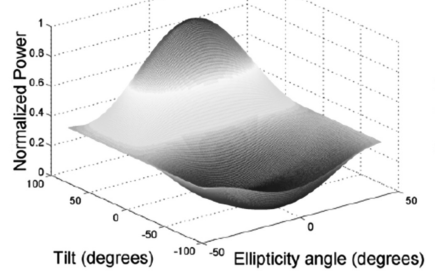

Cross-polarization signature of PARC2

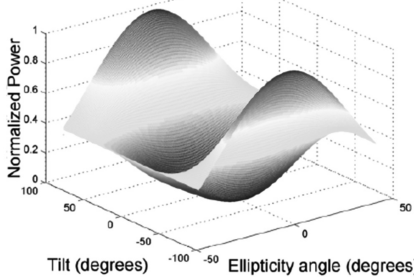

(b)

Co-polarization signature of PARC3

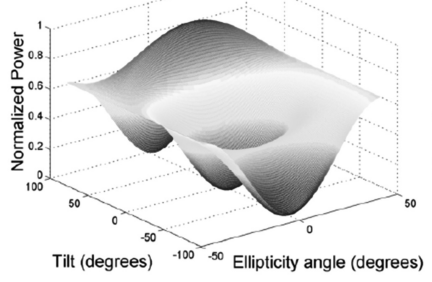

Cross-polarization signature of PARC3

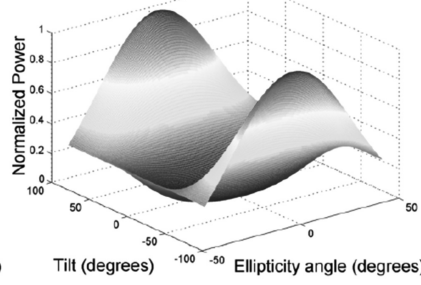

(c)

Co-polarization signature of Trihedral

Cross-polarization signature of Trihedral
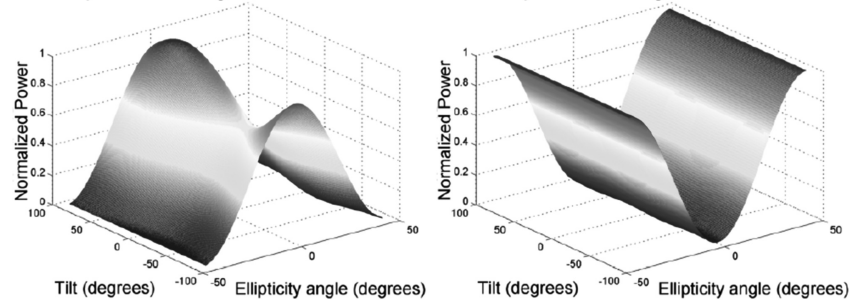

(d)

Fig. 20. Measured polarimetric signature of (a) PARC 1, (b) PARC 2, (c) PARC 3, and (d) trihedral corner reflector. scatterers have progressed considerably [14], [15]. The tomograms can be used, for instance, to monitor vegetated areas or to estimate ground topography. However, in order to retrieve the vertical scattering distribution, it is necessary to use multiple passes of the SAR sensor at different positions. In the airborne case, it is extremely difficult to obtain equally spaced baselines between the different passes. Besides, the flight paths can suffer deviations from the ideal straight trajectory in the $x$-, $y$-, and $z$-axis, which extremely complicates the processing of the data. In this context, the use of UAV MPs has several advantages that make it suitable to perform tomographic images. The possibility of this platform to perform vertical flights can be exploited to do vertical imaging in airborne SAR. With this strategy, the problems of the alignment and deviations of different flights might be

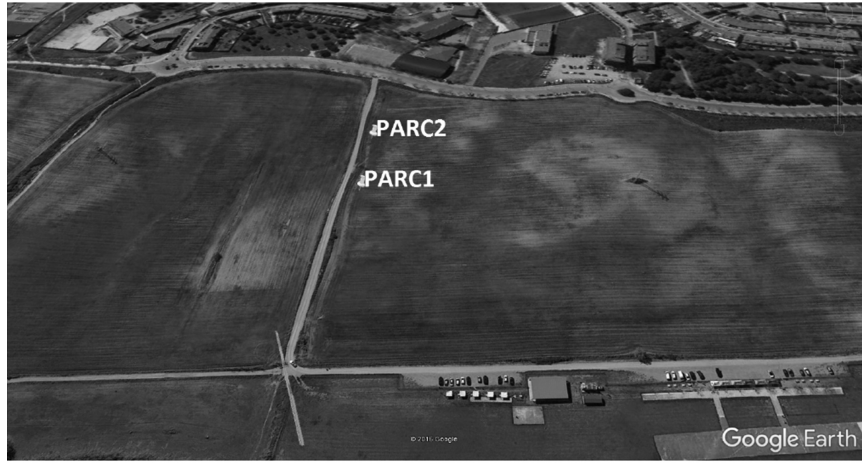

(a)

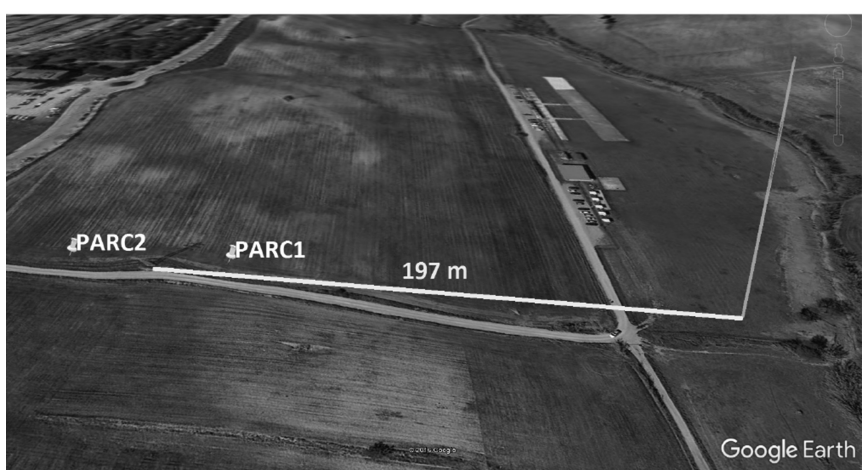

(b)

Fig. 21. Images of the scenario taken from Google Earth with the vertica flight path information. Ascending velocity of $2 \mathrm{~m} / \mathrm{s}$. (a) North-West orientation. (b) South-West orientation.

overcome. Fig. 21 shows two representations of a vertical flight path performed by the UAV MP taken from Google Earth. In our experience, the ascending vertical flight with the UAV MP is very stable and does not suffer from important deviations from the ideal flight path. Moreover, the ascending velocity of the platform is maintained almost constant, and the flight precision is equivalent to the horizontal stripmap acquisition mode.

In Fig. 21(b), a pylon is located at $197 \mathrm{~m}$ from the vertical flight trajectory. There are also two PARCs in the scenario at 173 and $226 \mathrm{~m}$. Moreover, the terrain of the scenario has an inclination of approximately $8 \%$ up to $300 \mathrm{~m}$, where the road separating the agricultural field from the city begins. Fig. 22(a) shows the SLC image of the scenario obtained with the ARBRES-X SAR sensor during a normal horizontal flight. The yellow line in the image corresponds to the vertical profile shown in Fig. 22(b), where the SLC obtained during the vertical flight is shown.

The pylon located at $197 \mathrm{~m}$ can be perfectly seen in Fig. 22(b) and also PARCs 1 and 2 at 173 and $226 \mathrm{~m}$, respectively. In the same way as in conventional SAR measurements, the resolution in elevation can be determined by (3). Table III summarizes the theoretical and measured resolutions for the 3-m aperture length. The theoretical azimuth resolutions for PARCs 1 and 2 are 1.48 and $1.96 \mathrm{~m}$, respectively, while the measured 3-dB azimuth resolutions are 1.93 and $2.08 \mathrm{~m}$. Again, considering the effect of the cross-range Hanning window, the measured resolutions are quite close to the theoretical ones. 


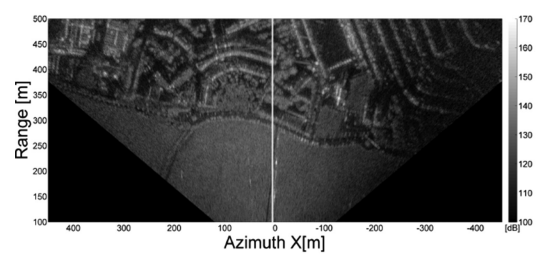

(a)

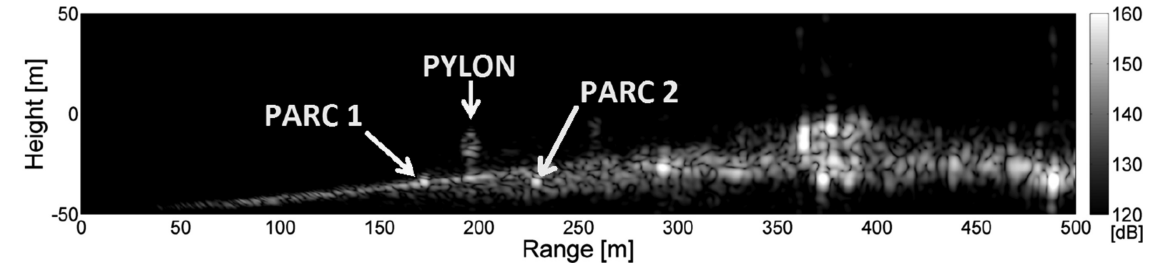

(b)

Fig. 22. (a) SLC image of the scenario during an horizontal aperture with flight conditions: 150-m height, $v_{\mathrm{UAV}}=8.9 \mathrm{~m} / \mathrm{s}$, VV Pol. (b) SLC image of the scenario during the vertical flight with flight conditions: $v_{\mathrm{UAV}}=2 \mathrm{~m} / \mathrm{s}, \mathrm{VV}$ Pol, $3-\mathrm{m}$ aperture length. The profile corresponds to the yellow line shown on the SLC image in (a).

TABLE III

AZIMUTH RESOLUTION OF THE VERTICAL FLIGHT

\begin{tabular}{lcc}
\hline \hline & $\Delta_{\mathrm{az}}$ Theoretical $[\mathrm{m}]$ & $\Delta_{\mathrm{az}}$ Measured $[\mathrm{m}]$ \\
\hline PARC 1 & 1.48 & 1.93 \\
PARC 2 & 1.96 & 2.08 \\
\hline \hline
\end{tabular}

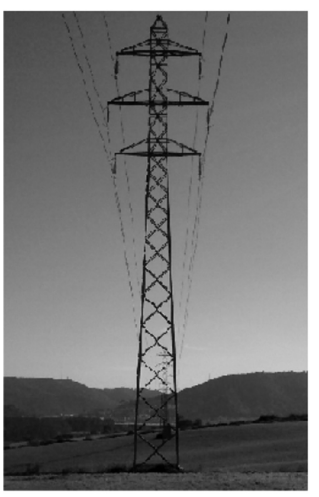

(a)

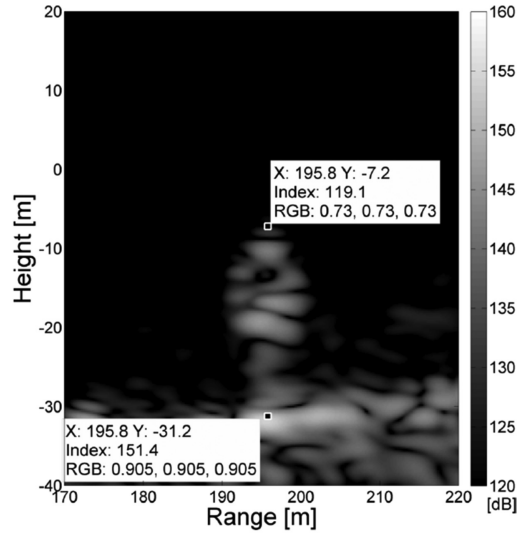

(b)
Fig. 23. (a) Photograph of the pylon in the scenario. (b) Zoom to the pylon in the vertical aperture of the scenario obtained with the ARBRES-X SAR sensor
Fig. 22(a) shows that the agricultural field of the scenario finishes approximately at $300 \mathrm{~m}$ and beyond this range distance starts the city. In Fig. 22(b), the contribution of all the scatterers covered by the antenna beamwidth corresponding to the urban area is collapsed at the same range. For that reason, there are a lot of bright pixels between 350 and $400 \mathrm{~m}$, which are the buildings of the city. The slope of the terrain derived from the vertical aperture is of approximately $8 \%$ up to $300 \mathrm{~m}$, what matches with the information obtained from topographic maps of the area. Fig. 23(a) shows a photograph of the pylon in the scenario, while Fig. 23(b) shows a zoom to the pylon observed in the vertical aperture of Fig. 22(b). The pylon has a height of $24 \mathrm{~m}$, which is comparable to the one obtained in the SLC image of Fig. 23(b), where the markers located at the base and the top of the pylon present a height difference of $24 \mathrm{~m}$.

In the way it is performed, the vertical image is affected by left-right ambiguity. Nevertheless, the goal of this experiment is to set the basis to vertical tomography, where this ambiguity is solved by considering a given number of vertical flights. In

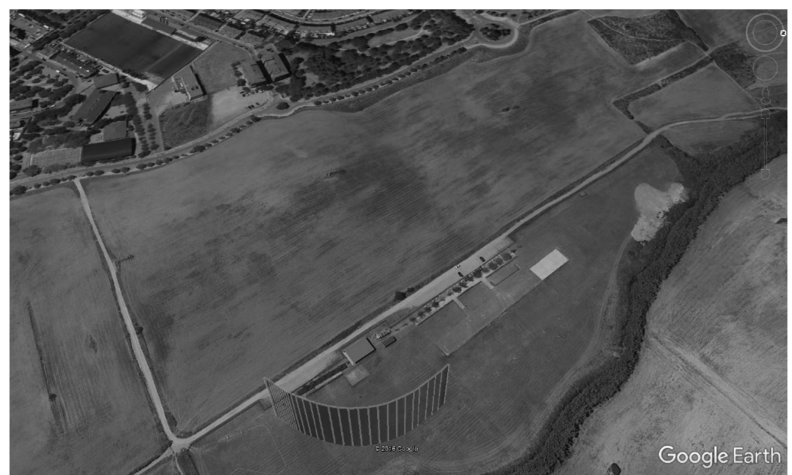

Fig. 24. Image of the scenario taken from Google Earth with the semicircular flight path information.

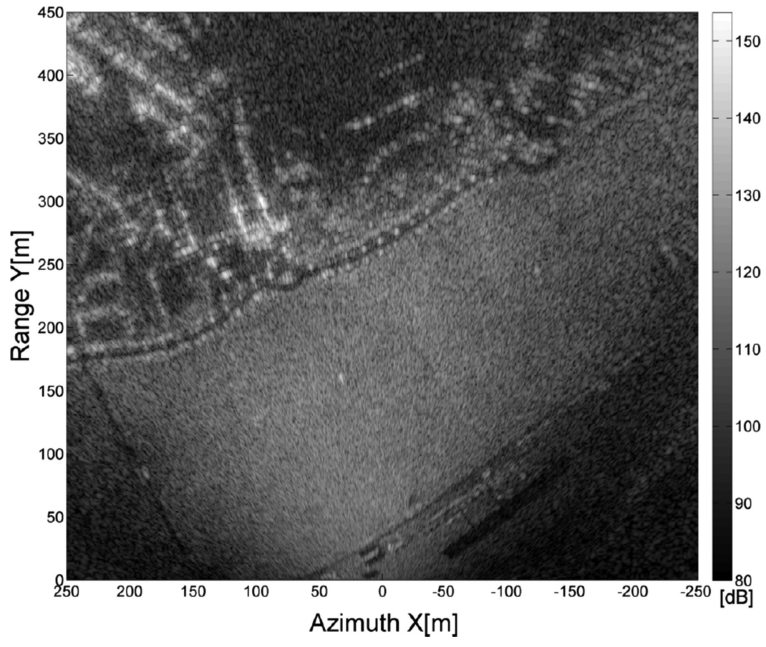

Fig. 25. SLC image of the scenario obtained with the ARBRES-X SAR sensor during the circular flight. Flight conditions: 50-m height, $v_{\mathrm{UAV}}=6.2 \mathrm{~m} / \mathrm{s}$, VV Pol

addition, in the experiment, this ambiguity has a minimum effect in the imaged tower as it comes from a grass area.

4) Circular Flight in UAV MP SAR: The possibility of the UAV MP to perform circular flights can also be exploited to do tomographic imaging in the future, the so-called circular SAR [15]. In this context, by acquiring different circular trajectories over an area, several options are possible [16]. The purpose of this section is to demonstrate the feasibility of acquiring 591 SAR data with circular trajectories of the UAV MP. As a first 592 approximation to these type of flights, a partial segment of a 593 
circular flight is processed in the present paper, which allows us to envisage the possibility to perform complete circular flights in the future.

Fig. 24 shows the flight path information of the partial segment of a circular flight taken from Google Earth, and Fig. 25 shows the SLC image of the scenario obtained with the ARBRES-X SAR sensor during this trajectory. The aperture length is approximately $9.3 \mathrm{~m}$, and the platform height is approximately $50 \mathrm{~m}$.

Theoretically, the advantage of obtaining SAR images with circular flights is that they allow the maximum attainable resolution of a fraction of the wavelength [15]. In the SLC image obtained with the circular flight in Fig. 25, the area that theoretically has better resolution corresponds to the constructions in the airfield, such as the different buildings or the landing strip.

\section{CONCLUSION}

In the present paper, the SAR capabilities of UAV MPs have been evaluated. In this context, the integration of the ARBRESX SAR sensor into a small multicopter platform has been presented, opening new possibilities in airborne remote sensing. The feasibility of the system has been demonstrated by performing several measurement campaigns in the RACBSA airfield. Furthermore, the main limitations and technical challenges of obtaining SAR data with this type of platforms have been assessed along the present study.

The capability of the UAV MP to execute different types of trajectories has been exploited to evaluate the system performance and to obtain first preliminary results. It has been observed that the defocusing problem in the retrieved images is due to the deviation of the platform from the nominal trajectory and the flight instabilities. To overcome this undesired effect, it has been concluded that the use of a cost-effective small-size IMU is a nonviable option. This is because of the lack of accuracy of these systems, which has forced us to discard the use of a MoCo technique to focus the image. Alternatively, an autofocus algorithm has been applied to refocus the SLC images, improving its quality for large apertures.

Otherwise, the inherent instability of the platform and the insufficient positioning accuracy of the GPS system make it extremely difficult to obtain interferometric results. The autofocus algorithm is well suited to compensate amplitude images, but the problem is not yet efficiently resolved with the phase in long apertures. Because of that, the interferometric measurements have been processed using small apertures, where the flight deviations are less important. Nevertheless, the first SAR interferogram obtained with a repeat-pass flight performed with a UAV MP has been presented.

On the other hand, a vertical and a partial segment of a circular aperture have been successfully performed obtaining SLC images of the scenario, which envisages the capability of the UAV MP to perform tomographic images and complete circular apertures in the future. Regarding the vertical flight, it has been observed that the ascending velocity of the platform is very stable and the trajectory does not suffer from important deviations from the nominal track.
One of the limitations of ground-based SAR (GB-SAR) sensors is its dependence on the geometry of the scenario to illuminate the area under study. Sometimes, having the possibility to find the optimal position to place the GB-SAR sensor with the required orientation can be a problem. In the future, the use of UAV MPs can overcome this drawback allowing the monitoring of almost any site. In this sense, the use of SAR sensors integrated in UAV MPs can be considered as an interesting alternative between GB-SAR and airborne SAR sensors.

In conclusion, the UAV MP is a promising platform that opens new potentials for several applications, such as repeatpass interferometry or differential tomography imaging with the realization of almost arbitrary trajectories.

\section{REFERENCES}

[1] J.-S. Lee and E. Pottier, Polarimetric Radar Imaging: From Basics to Applications. Boca Raton, FL, USA: CRC Press, 2009, p. 440. [Online]. Available: http://www.crcnetbase.com/doi/abs/10.1201/ 9781420054989.fmatt

[2] J. C. Curlander and R. N. McDonough, Synthetic Aperture RadarSystems and Signal Processing. Hoboken, NJ, USA: Wiley, 1991.

[3] R. F. Hanssen, Radar Interferometry - Data Interpretation and Error Analysis, vol. 2. New York, NY, USA: Springer, 2001. [Online]. Available: http://link.springer.com/10.1007/0-306-47633-9

[4] A. Aguasca, R. Acevo-Herrera, A. Broquetas, J. J. Mallorqui, and X. Fabregas, "ARBRES: Light-weight CW/FM SAR sensors for small UAVs," MDPI, vol. 13, no. 3, pp. 3204-3216, 2013.

[5] R. Acevo-Herrera, A. Aguasca, J. J. Mallorqui, and X. Fabregas, "Highcompacted FM-CW SAR for boarding on small UAVS," in Proc. Int Geosci. Remote Sens. Symp., 2009, vol. 2, pp. II-543-II-546.

[6] R. J. C. Middleton, "Dechirp-on-receive linearly frequency modulated radar as a matched-filter detector," IEEE Trans. Aerosp. Electron. Syst., vol. 48, no. 3, pp. 2716-2718, Jul. 2012.

[7] M. Soumekh, Synthetic Aperture Radar Signal Processing with MATLAB Algorithms. Hoboken, NJ, USA: Wiley, 1999. [Online]. Available: http://www.amazon.com/Synthetic-Aperture-SignalProcessing-Algorithms/dp/0471297062

[8] D. E. Wahl, P. H. Eichel, D. C. Ghiglia, and C. V. J. Jakowatz, "Phase gradient autofocus-A robust tool for high resolution SAR phase correction," IEEE Trans. Aerosp. Electron. Syst., vol. 30, no. 3, pp. 827-835, Jul. 1994.

[9] B. Fan, Z. Ding, W. Gao, and T. Long, "An improved motion compensation method for high resolution UAV SAR imaging," Sci. China Inf. Sci., vol. 57, no. C, pp. 1-13, 2014.

[10] A. Alabort and J. J. Mallorqui, "Development of motion compensation algorithms for airborne UAV SAR," 2014

[11] P. Rosen et al., "Synthetic aperture radar interferometry," Proc. IEEE, vol. 88, no. 3, pp. 333-382, Mar. 2000. [Online]. Available: http:// ieeexplore.ieee.org/lpdocs/epic03/wrapper.htm?arnumber $=838084$

[12] F. Gatelli, A. M. Guarnieri, F. Parizzi, P. Pasquali, C. Prati, and F. Rocca, "The wavenumber shift in SAR interferometry," IEEE Trans. Geosci. Remote Sens., vol. 32, no. 4, pp. 855-865, Jul. 1994.

[13] A. Reigber, P. Prats, and J. J. Mallorqui, "Refined estimation of timevarying baseline errors in airborne SAR interferometry," in Proc. Int Geosci. Remote Sens. Symp., 2005, vol. 7, pp. 4799-4802.

[14] A. Reigber and A. Moreira, "First demonstration of airborne SAR tomography using multibaseline L-band data," IEEE Trans. Geosci. Remote Sens., vol. 38, no. 5 I, pp. 2142-2152, Sep. 2000.

[15] A. Moreira, P. Prats-iraola, M. Younis, G. Krieger, I. Hajnsek, and K. P. Papathanassiou, "A tutorial on synthetic aperture radar," IEEE Geosci. Remote Sens. Mag., vol. 1, no. 1, pp. 6-43, Mar. 2013. [Online]. Available: http://ieeexplore.ieee.org/lpdocs/epic03/wrapper.htm?arnumber= 6504845

[16] O. Ponce, P. Prats-Iraola, R. Scheiber, A. Reigber, A. Moreira, and E. Aguilera, "Polarimetric 3-D reconstruction from multicircular SAR at Pband," IEEE Geosci. Remote Sens. Lett., vol. 11, no. 4, pp. 803-807, Apr. 2014. 


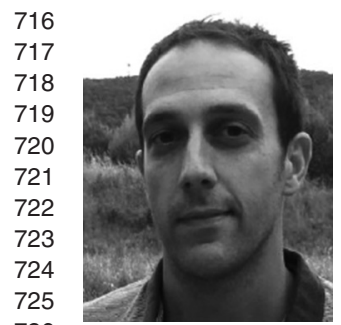

Marc Lort was born in Sabsadell, Spain, in 1986. He received the B.Sc. degree in telecommunication engineering from the Universitat Autònoma de Barcelona, Barcelona, Spain, in 2010. In 2013, he joined the Signal Theory and Communications Department, Universitat Politècnica de Catalunya (UPC), Barcelona, and received the M.Sc. degree in 2014. He is currently working toward the Ph.D. degree, focused on multidimensional synthetic aperture radar (SAR) for the Earth observation, with UPC.

His main research interests include the development and test of SAR systems integrated in unmanned aerial vehicles.

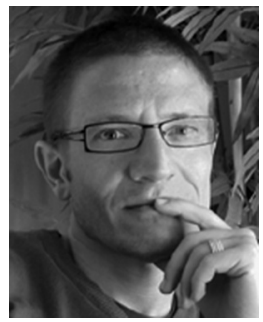

Carlos López-Martínez (S'97-M'04-SM'11) re- 744 ceived the M.Sc. degree in electrical engineering and 745 the Ph.D. degree from the Universitat Politècnica de 746 Catalunya, Barcelona, Spain, in 1999 and 2003, re- 747 spectively.

From October 2000 to March 2002, he was with the Frequency and Radar Systems Department, German Aerospace Center (DLR), Oberpfaffenhofen, Germany. From June 2003 to December 2005, he was with the Image and Remote Sensing GroupSAPHIR Team, Institute of Electronics and Telecommunications of Rennes (I.E.T.R. CNRS UMR 6164), Rennes, France. In January 2006, he joined as a Ramn-y-Cajal Researcher the Universitat Politècnica de Catalunya, Barcelona, Spain, where he is currently an Associate Professor in the area of remote sensing and microwave technology. He has organized different invited sessions in international conferences on radar and synthetic aperture radar (SAR) polarimetry. He has presented advanced courses and seminars on radar polarimetry to a wide range of organizations and events. He has authored or coauthored more than 100 articles in journals, books, and conference proceedings in the radar remote sensing and image analysis literature. His research interests include SAR and multidimensional SAR, radar polarimetry, physical parameter inversion, digital signal processing, estimation theory, and harmonic analysis.

Dr. López-Martínez is an Associate Editor of the IEEE JOURNAL OF SELECTED TOPICS IN APPliEd EARTH OBSERVATIONS AND REMOTE SENSING. He served as a Guest Editor of the EURASIP Journal on Advances in Signal Processing. He received the Student Prize Paper Award at the EUSAR 2002 Conference and coauthored the paper awarded with the First Place Student Paper Award at the EUSAR 2012 Conference. He has also received the IEEE-GRSS 2013 GOLD Early Career Award.

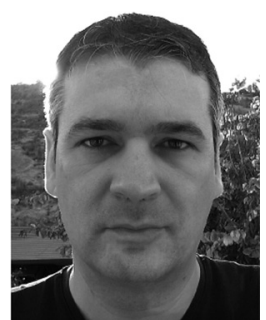

Tomás Martínez Marín received the Tech.Eng. 775 (B.S.) degree from the University of Alcalá, Alcalá 776 de Henares, Spain, in 1990, and the M.S. and Ph.D. 777 degrees in telecommunications engineering from the 778 Technical University of Madrid, Madrid, Spain, in 779 1995 and 1999, respectively .

He joined the University of Alcalá as an Assistant Professor in 1990. In 1997, he joined the European University of Madrid as an Assistant Professor. Since 2000, he has been with the Department of Physics, System Engineering and Signal Theory, University of Alicante, Alicante, Spain, where he is currently an Associate Professor. His research interests include reinforcement learning, intelligent vehicles, simultaneous localization and mapping, polarimetric and interferometric techniques, and synthetic aperture radar imaging algorithms.
Albert Aguasca (S'90-M'94) was born in Barcelona, in 1989 and 1993, respectively. UPC. His teaching activities involve radio-frequency and microwave circuits for communications and radio navigation systems. He has authored or coau-
749

750

751

752

753

754

755

756

757

758

759

760

761

762

763

764

765

766

767 
- Authors: When accessing and uploading your corrections at the Author Gateway, please note we cannot accept new source files as corrections for your paper. Do not send new Latex, Word, or PDF files, as we cannot simply "overwrite" your paper. Please submit your corrections as an annotated PDF or as clearly written list of corrections, with location in paper. You can also upload revised graphics to the Gateway.

- Authors: Please note that once you click "approve with no changes," the proofing process is now complete and your paper will be sent for final publication and printing. Once your paper is posted on Xplore, it is considered final and the article of record. No further changes will be allowed at this point so please ensure scrutiny of your final proof.

- Authors: Unless invited or otherwise informed, a mandatory Excessive Paper Length charge of $\$ 200.00$ per page (beginning with page 7 and beyond) is required for papers in excess of six (6) printed pages for JSTARS. If you have any questions regarding overlength page charges, need an invoice, or have any other billing questions, please contact reprints@iee.org as they handle these billing requests.

Q1. Author: Please provide full bibliographic details in Ref. [10].

Q2. Author: Please provide the subjects in which the author Carlos López-Martínez received the Ph.D. degree.

Q3. Author: Please provide the subjects in which the author Tomás Martínez Marín received the Tech.Eng. (B.S.) degree. 